\title{
„PHASEN“ - „PERIODEN“ - „ZEITSCHEIBEN“ \\ ZUR CHRONOLOGIE DER SPÄTKUPFERZEITLICHEN NEKROPOLEN IM KARPATENBECKEN
}

\author{
MÁRIA BONDÁR - ISTVÁN ZALAI-GAÁL (†) \\ Forschungszentrum für Humanwissenschaften \\ der Ungarischen Akademie der Wissenschaften \\ Archäologisches Institut \\ Tóth Kálmán u. 4, H-1097 Budapest, Ungarn \\ bondar.maria@btk.mta.hu
}

\begin{abstract}
This study investigates the relative chronology of the Late Copper Age Baden culture by analysing the pottery of the largest known cemeteries (Alsónémedi, Budakalász, Fonyód-Bézsenypuszta, Balatonlelle-Felső Gamász, Pilismarót-Basaharc and Mezöcsát-Hörcsögös). Altogether 611 ceramic finds from 253 graves were involved in the research. The results presented here are preliminary; all the known Baden cemeteries will be processed in the future.

Keywords: Late Copper Age, Baden culture, cemetery chronology, metrical pottery analysis
\end{abstract}

\section{EINLEITUNG}

Als Übergang zwischen der mittleren Kupferzeit und der Spätkupferzeit werden die Furchenstichkeramik und das Protoboleráz auch durch die jüngsten Forschungen bestimmt. C. Sachße bestätigt, dass sich die Gruppen der Kultur mit Furchenstichkeramik etwa um 3800 v. Chr. aus den Epi-Lengyel-Varianten im westlichen Karpatenbecken formieren. „Verbunden damit ist eine verstärkte gegenseitige Annäherung des westlichen und östlichen Kulturraumes im Karpatenbecken... Dies mündet etwa um 3600 v. Chr. in den Protoboleráz-Horizont als Initialphase der Badener Kultur, der sowohl große Teile des Gebietes der FSK als auch das der Hunyadi-halom-Kultur im Theiß-Gebiet umspannt.“1

Bei der Herausbildung der erwähnten Boleráz-Gruppe haben die früheren Forschungen die Rolle des sog. „Protoboleráz-Horizontes“ betont. V. Nĕmejcová-Pavúková anerkannte die Selbstständigkeit dieses Horizontes und sie nannte die Übergangsphase Boleráz Ia-Phase. ${ }^{2}$ Die Fragen der Chronologie, Typologie und Verbreitung des Protoboleráz-Horizontes erörterte N. Kalicz ausführlich und bestätigte, dass die Begegnung von zwei Kulturräumen in diesem neuen Horizont beobachtet werden kann. Zweifellos überwiegen die aus dem Südosten stammenden Komponenten, sie bestimmen das Bild des Protoboleráz-Horizontes. ${ }^{3}$ In der Interpretation der Cernavoda III-, Protoboleráz- und Boleráz-,,Erscheinungen“ kann eine Unsicherheit auf Grund des derzeitigen Forschungsstandes festgestellt werden. Auch die Terminologien, Meinungen sind verschieden. M. Bondár stimmt den Interpretationen zu, nach denen Cernavoda III und Boleráz zwei unterschiedliche Kulturen darstellen, die sich aus verschiedenen Grundlagen herausgebildet hatten. ${ }^{4}$

Die Ergebnisse der von Sz. Fábián und ihren Mitarbeitern vorgenommenen Untersuchungen an den Fundstellen von Abony $49^{5}$ und Abony 36 im Verhältnis zur Sequenz von Balatonkeresztúr datieren das „Protoboleráz-

\footnotetext{
I SACHßE 2009, 155.

${ }^{2}$ NĚMEJCOVÁ-PAVÚKovÁ 1964, 51; NĚMEJCOVÁ-PAVÚKOVÁ 1979.
}

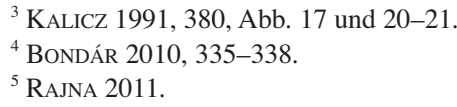

${ }^{3}$ KaLICZ 1991, 380, Abb. 17 und 20-21.

${ }^{4}$ BONDÁR 2010, 335-338.

${ }^{5}$ RAJNA 2011. 
Phänomen“ in die Zeit von 4432-4268/4334-4161 cal. BC bis 3191-2949/3037-2817 cal. BC und sie werfen die Frage auf: „Is the name Protoboleráz correct?“6

M. Furholt stellte in seiner Arbeit über die Keramikstile der Badener Kultur im spätneolithischen Kontext fest, dass „Mitteleuropa im 4. Jahrtausend, besonders im südlichen Teil, von kleinräumigen archäologischen Klassifikationseinheiten, archäologischen Kulturen dominiert zu sein scheint" ${ }^{.7}$ Die Fragen und Probleme der nördlichen Badener Keramikstile betrachtete er im Kontext des mitteleuropäischen Spätneolithikums. ${ }^{8}$

Die Badener Kultur ist in erster Linie auf Grund der Keramik definiert. M. Furholt hält zugleich fest, ,,dass die Badener Kultur nichts anderes darstellt als eine Reihe vergesellschafteter keramischer Typen. Diese sollen zunächst als Stil bezeichnet werden, dessen Einheitlichkeit zu hinterfragen ist. “9

\section{ARBEITSRAUM UND FUNDGESCHICHTE}

In der vorliegenden Arbeit wird versucht, die relative chronologische Stellung der größten bekannten Gräberfelder der Badener Kultur sowie die möglichen chronologischen Beziehungen zwischen diesen Nekropolen (Alsónémedi, Budakalász, Fonyód-Bézsenypuszta, Balatonlelle-Felső Gamász, Pilismarót-Basaharc und Mezőcsát-Hörcsögös) zu klären. Es handelt sich um die Präliminarien zu einer Analyse aller bekannten Nekropolen der Badener Kultur.

J. Korek war der Meinung, dass das extramurale Gräberfeld von Alsónémedi „,mit 40 Gräbern als erschöpft bezeichnet werden kann. Dieses kleinere Gräberfeld bestand zeitgleich zum jüngeren Abschnitt von Budakalász.“10 „Kleine Unterschiede sind chronologisch wie auch mikroregional bedingt: Hier liegen ausschließlich Körpergräber ohne Steinsetzung vor" - stellte C. Sachße fest. Er datierte die Befunde in die entwickelte klassische Stufe der Badener Kultur. ${ }^{11}$

Das extramurale Gräberfeld von Budakalász-Luppa csárda umfasst 436 Brand- und Körperbestattungen sowie symbolische Gräber. ${ }^{12}$ Von diesen Befunden, die in den Jahren zwischen 1952 und 1960 ausgegraben wurden, veröffentlichte M. Bondár die Funde von 115 Gräbern. ${ }^{13}$ C. Sachße ist der Meinung, dass die Belegung hier mit Brandgräbern unter Steinabdeckung beginnt, ,,wie man es vom frühen Baden in dieser Region kennt“. ${ }^{14}$ Festgestellt werden kann aber, dass man die Angaben zur Zahl der Bestattung in der Literatur leicht differenzieren kann. ${ }^{15}$ Neue Daten weisen schon auf 436 Grabbefunde mit 468 Individuen und 355 Körpergräber, 70 Brandgräber, eine birituelle Bestattung, acht symbolische Gräber sowie ein leeres Grab hin. „Auch konnte die Nordgrenze des Gräberfeldes nicht erreicht werden. Daher rechnet man mit einer tatsächlichen Existenz von ca. 500 Gräbern, womit ca. 85-90 \% des Gräberfeldes erschlossen wurde. “16 C. Sachße datiert die publizierten Gräber in das entwickelte klassische

\footnotetext{
${ }^{6}$ FÁBIÁN et al. 2015: „The 'Protoboleráz' material probably appears at lot more sites in the Carpathian Basin, as we know but it is difficult to recognise; Typologically heterogeneous, it is a transition from the Middle to the Late Copper Age (in Hungary); Ceramic technology is also heterogeneous then it became homogenous; Symbolic activities had connections to both the Middle and the Late Copper Ages; Network of raw materials of chipped stones covered a more closed area than the previous periods. This tendencies seems to be stronger during the Late Copper Age. Chronologically it fits perfectly between the Middle Copper Age cultures and the Boleráz (Baden).“

7 „Die gesamte Badener Kultur lässt sich durch eine polythetische Betrachtungsweise leicht dekonstruieren. Es gibt keine weitere Material- bzw. Datenkategorie, die der Keramikverbreitung ähnliche Grenzen zeigt" (FurHOLT 2011, 246).

${ }^{8}$ FURHOLt 2013.

9 ,The so-called Baden culture does not embrace a consistent cultural package, and even if expressed by pottery alone has been shown here to be a coarse approximation of a number of ceramic subsystems“ (FURHOLT 2008a, 627). „Der Badener Keramikstil oder besser die Badener Keramikstile sind als kulturelle Standardisierungen auf einer konkreten Standardisierungsebene - der Gefäßkeramik - aufzufassen und in ihrem Zusammenspiel und ihrer Interaktion mit ande-
}

ren Standardisierungen auf derselben Ebene und in ihrem Verhältnis zu Standardisierungen auf anderen vergesellschafteten Ebenen zu untersuchen. Dies kann nur gelingen, wenn die zeitliche Ordnung mittels typologieunabhängiger Methoden erfolgt“ (FURHOLT 2009, 22, 26).

${ }^{10}$ KOREK 1951, 42.

${ }^{11}$ SACHßE 2009, 161; SACHßE 2010, 3, Taf. 10.

${ }^{12}$ MRT 7, 1986, 45; Soproni 1956. Die Beschreibung der Nekropole stellte J. Banner auf Grund der Briefe und Berichte zusammen, die Gy. Bacsó für das Ungarische Nationalmuseum (UNM) geschickt hatte (Archiv 19/1938. des Nationalmuseums). Die Fotoaufnahmen stammen aus dem Jahre 1940. Die Funde befinden sich in dem Ungarischen Nationalmuseum bzw. im Balaton-Museum von Keszthely. Ein Teil der Funde wurde während des Weltkriegs vernichtet. Im UNM sind Funde von 21 Gräbern zu finden; die Gräber 1-7, 9, 18-19 und 21 sind spätkupferzeitliche Urnengräber.

${ }^{13}$ BONDÁR 2009a; BONDÁR 2009b.

${ }^{14}$ SACHßE 2009, 158.

${ }^{15}$ NeVIZANSKY 1985, 252; MRT 7, 45, 47; BondÁR 1990/91, 36; SACHßE 2010, 17-18.

${ }^{16}$ MRT 7, 45; BondÁr 2009a; BONDÁR 2009b; SACHßE 


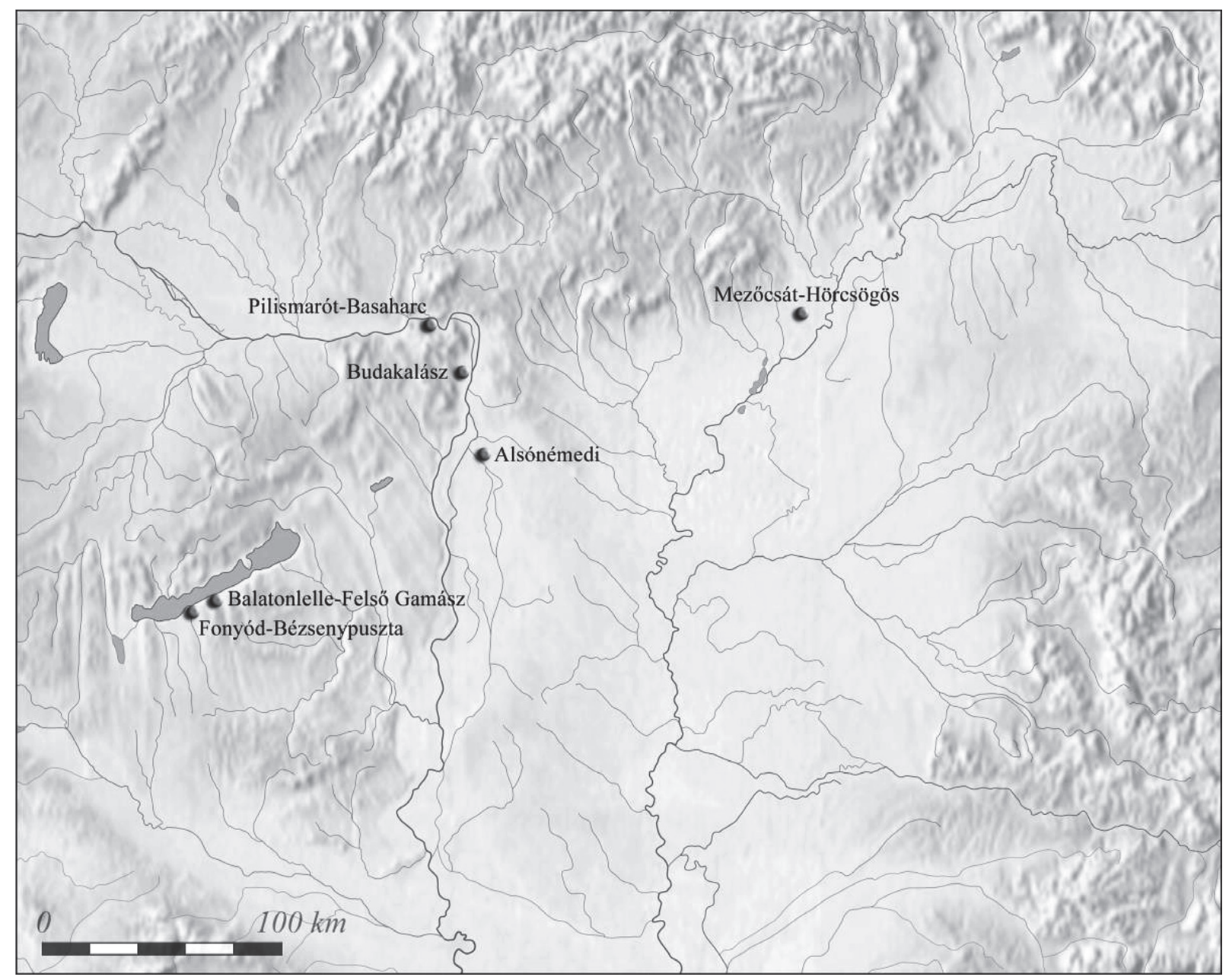

Abb. 1. Die untersuchten spätkupferzeitlichen Gräberfelder im Karpatenbecken

Baden. Der später ausgegrabene Bereich repräsentiert dagegen den älteren Abschnitt, in der Frühstufe des klassischen Baden beginnend. ${ }^{17}$

Im südlichen Transdanubien kennen wir nur wenige kleine Körpergräbergruppen am Balaton. Im Brandgräberfeld Fonyód-Bézsenypuszta mit 11 Bestattungen ${ }^{18}$ fand man 8 Urnenbestattungen und eine Brandschüttung bzw. zwei Gräber von unsicherem Charakter, die verschiedene Zeitstufen vertreten und in die späte Boleráz-Stufe datieren. ${ }^{19}$ C. Sachße behauptet, dass das keramische Material hier dem Boleráz-Stil recht nahe steht und „es ist vergleichbar mit dem zwischen Cluster 1 und 3/4 eingeordneten Komplex von Tekovskýy Hrádok. Deshalb spricht Němejcová-Pavúková vom Horizont Fonyód-Tekovskýy Hrádok. “20 I. Torma bestimmte diesen als der späteste Boleráz-Abschnitt. ${ }^{21} \mathrm{C}$. Sachße geht deshalb also auch für diese Region von einer gewissen Tradierung der BolerázElemente in Kontexten aus, die bereits mit der klassischen Badener Keramik vergesellschaftet sind. Wenn auch die Datenbasis recht klein ist, so lassen sich doch keine klaren Unterschiede innerhalb Transdanubiens aufzeigen. Wohl etwas später datieren solche Gräber, die Kostolac-Einflüsse zeigen. ${ }^{22}$ Dieses Gräberfeld, „das am Übergang zum klassischen Baden steht“ ${ }^{23}$ brachte auch M. Furholt mit der Boleráz-Keramik in Verbindung.

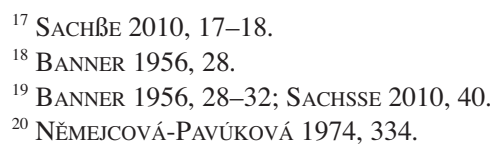

\footnotetext{
${ }^{21}$ TORMA $1973,498$.

${ }^{22}$ SACHßE 2005.

${ }^{23}$ FURHOLT 2009, 130.
} 
Auf dem Fundplatz Balatonlelle-Felső Gamász wurden 22 (23) Gräber mit Körperbestattungen außerhalb einer frühbadenzeitlichen Siedlung entdeckt. ${ }^{24}$ Diese Befunde datierte A. Sófalvi in die klassische Badener Kultur. C. Sachße ordnete sie zeitlich dagegen der Zeitspanne von der Boleráz-Stufe bis die jüngere klassische Stufe der Badener Kultur zu. ${ }^{25}$

Südlich von der Theiß-Bodrog-Mündung wurde das birituelle Gräberfeld Mezőcsát-Hörcsögös in den Jahren 1958 bis 1962 von E. Patek und N. Kalicz ausgegraben. Es handelt sich dabei um sechs Körperbestattungen und acht (neun) Brandschüttungen. ${ }^{26}$ Hier und auch in Tiszavasvári ${ }^{27}$ befinden sich birituelle Gräbergruppen mit Körper-, Brandschüttungs- und Urnengräbern. Im südlichen Teil der Ungarischen Tiefebene sind nur wenige Bestattungen vorhanden, die sich am besten mit den transdanubischen vergleichen lassen. ${ }^{28} \mathrm{C}$. Sachße datierte das Gräberfeld von Mezőcsát-Hörcsögös in die entwickelte bis späte klassische Stufe der Badener Kultur. ${ }^{29}$

Die Ausgrabungen von Pilismarót-Basaharc wurden in den Jahren 1959 bis 1972 am Donauknie durchgeführt und sind mit dem Namen von István Torma verbunden. Über die Verbreitung der Boleráz-Gruppe stellte er einen Fundortkataster mit Funden von 100 Fundorten zusammen. ${ }^{30}$

Die Funde und Befunde von 116 Gräbern dieses extramuralen Gräberfeldes veröffentlichte jüngst M. Bondár. ${ }^{31}$ Die Keramik vertritt die Boleráz-Gruppe: C. Sachße datiert die Gräber in die entwickelte und späte Boleráz-Stufe und vereinzelt in die frühklassiche Stufe der Badener Kultur. ${ }^{32}$ Im Bereich des Donauknies liegen wenige isolierte Bestattungsplätze mit Brandschüttungs- und Urnengräbern unter Erdaufschüttungen oder Steinsetzungen vor. ${ }^{33}$

Über die geographische Lage der untersuchten Gräberfelder kann man zusammenfassend Folgendes resümieren. Die Fundorte von Balatonlelle-Felső Gamász und Fonyód-Bézsenypuszta liegen am Südufer des Balaton, einander nahe, im mittleren Bereich Transdanubiens. Pilismarót-Basaharc und Budakalász befinden sich im Donauknie in Nordungarn. Der Fundplatz von Alsónémedi platziert dagegen im nordwestlichen Teil der Großen Ungarischen Tiefebene. Das Gräberfeld von Mezőcsát-Hörcsögös liegt von den anderen weit entfernt, im östlichen Bereich der Tiefebene. Diese Fundorte können demnach drei oder vier Mikroregionen zugeordnet werden (Abb. 1).

\section{ZIELSETZUNG UND METHODEN}

Wir können M. Furholt und Ph. Stockhammer zustimmen, dass „,die Grundlage einer jeden archäologischen Betrachtung die räumliche und zeitliche Ordnung des archäologischen Fundgutes ist, die es überhaupt erst ermöglicht, Regelhaftigkeiten zu erkennen. Wiederholt belegte, spezifische Fundumstände, etwa die regelmäßige Beigabe einer bestimmten Gefäßform ins Grab oder die regelhafte Kombination von Funden wie z. B. bestimmten Bronzeformen in Hortfunden, verweisen auf den intentionalen Umgang und damit auch auf die Wahrnehmung von uns entsprechend gedeuteter Formen bzw. Verzierungen von Objekten als Zeichen. “ ${ }^{* 34}$

Im Fall der vorliegenden Arbeit handelt es sich um die formanalytische Auswertung aller in die Datenbasis aufgenommenen Keramiken der untersuchten spätkupferzeitlichen Nekropolen im klein- und interregionalen Kontext. Bei diesen Gräbern handelt es sich um geschlossene Befunde, in denen absichtlich und gleichzeitig deponierte Funde vorhanden sind. ${ }^{35}$ Ebenso wie im Fall der zitierten Arbeiten von M. Furholt, nimmt die Analyse der keramischen Merkmale den größten Anteil auch in der vorliegenden Arbeit ein. ${ }^{36}$

${ }^{24}$ SÓFALVi 2004; NAGY 2010, 375.

${ }^{25}$ SÓFAlVi 2004; SACHßE 2009, 158; SACHßE 2010, 13. Das Grab str. 142 (Sófalvi 2004, Taf. 6.1) erscheint in der Publikation von B. Nagy als Grab 22 (NAGY 2010, 480-487) und die Objekt str. 496 (Sófalvi 2004, Taf. 6.2) als Grab 6 (NAGY 2010, 432-436).

${ }^{26}$ KaLiCZ 1999, 101.

${ }^{27}$ NEVIZÁNSKY 1985, 267.

${ }^{28}$ FURHOLT 2009, 133.

${ }^{29}$ SACHßE 2010, 63-64.

${ }^{30}$ ToRma 1969, 91-100. Die Ausgrabungen führte hier N. Fettich im Jahre 1959 durch (drei Badener Bestattungen). Die Grabungsleitung übernahm dann I. Torma in den Jahren von 1962 bis 1967 und 1972.

${ }^{31}$ TORMA 1973; BONDÁR 2015.

${ }^{32}$ SACHßE 2010, 87

33 TORMA 1973.

${ }^{34}$ FurholT-STOCKHAMmer 2008, 61.

35 „Die Bestattungssitten stellen eine Standardisierungsebene dar, die in der archäologischen Forschung deswegen immer eine herausragende Bedeutung spielt, weil sie in Menge und Qualität der Quellen den Siedlungen mindestens gleichwertig ist. Man kann davon ausgehen, dass Bestattungssitten eng mit dem rituell-religiösen Bereich menschlicher Kultur verknüpft bzw. in der Regel ein Ausdruck dieses Bereiches sind. Daher handelt es sich also um eine Standardisierungsebene, die einerseits wohl mehr als der Bereich der Siedlungsweise von kulturellen Standardisierungen bestimmt und andererseits weniger als dieser von natürlichen Bedürfnissen determiniert wird“ (FuRHOLT 2009, 129-130). 
Die Forschungskonzepte und Möglichkeiten der Analyse von prähistorischen Gefäßverzierungen wurden unter anderen von P. Biehl und R. Gleser ausführlich erörtert. ${ }^{37}$ Dem Thema „Keramik als chronologisches Indiz und ethnischer Indikator" widmete auch A. Zeeb-Lanz mehrere Studien. ${ }^{38}$ Die kontextuelle Merkmalanalyse der prähistorischen Keramiken setzt selbstverständlich die Untersuchung aller erfassbaren Merkmale der Artefakte voraus. M. Furholt errechnete für die badenzeitliche Gefäßkeramik eine überregionale Korrespondenzanalyse der Ziermotive, die ,gut mit den Ergebnissen einer Reihe regionaler Einzelanalysen harmoniert“ ${ }^{39}$ Die Untersuchung der Verzierungen aller in die Analyse aufgenommenen Keramiken auf Grund der Fotoabbildungen war für uns nicht möglich. Die zeitlichen und räumlichen Änderungen der Grabkeramik der behandelten Gemeinschaften untersuchten wir anhand der Profilierung der Funde.

Das Ziel der vorliegenden Arbeit können wir folgenderweise zusammenfassen. 1. Die Klassifikation des grabkeramischen Bestandes der untersuchten Nekropolen der Badener Kultur, um die möglichen typochronologischen Einheiten innerhalb unseres Systems bestimmen zu können. 2. Zur Klassifikation der Grabkeramiken der Gräberfelder der Badener Kultur wurden die archäologische vergleichende Methode, die hierarchisch aufgebaute metrische Analyse (mAnalyse), ferner die Korrelation und Seriation (WINBASP 5.2) verwendet. 3. Bestimmung der Tendenzen der Belegungsgeschichte der untersuchten Nekropolen nach Belegungsphasen (,,künstlichen Perioden") im Verhältnis von Radiokarbondaten.

Die Klassifizierung der Funde und Befunde nahmen wir mit Hilfe eines hierarchisch aufgebauten merkmalanalytischen Systems vor, das auch schon früher, bei der Bearbeitung der lengyelzeitlichen Grabkeramik und der sozialarchäologischen Verhältnisse von neolithischen und kupferzeitlichen Gräberfeldern, angewendet wurde. ${ }^{40}$ Somit erhalten wir eine Reihenfolge von Funden und Befunden, die sich nach einem statistischen oder typologischen Bild ordnen. Es muss aber entschieden bzw. nachgewiesen werden, ob dieses Bild auch die relativchronologische Reihenfolge der untersuchten Objekte spiegelt oder eben nicht spiegelt. ${ }^{41}$ Wir berücksichtigten nur Merkmale, die für unsere Fragestellung als relevant gelten.

Der Umstand, dass genügend Daten nicht immer für jede Materialkategorie in jedem Gräberfeld und für jede Periode vorliegen, stellt auch im Fall unserer metrischen Analyse ein methodisches Problem dar. Die Datenbasis für die Badener Keramikstile benutzt M. Furholt als eine sehr kleinteilige Merkmalkodierung: „Im Zuge einer polythetischen Klassifikation des Materials stellt die Gefäßkeramik eine Datenkategorie dar, die jedoch mit wesentlich wenigeren kleinteilig klassifizierten Artefaktskategorien zu vergleichen ist, wenn man die Frage nach stilistischer Varianz im Raum beantworten will.“42

\section{ZU DEN CHRONOLOGISCHEN FORSCHUNGEN DER BADENER KERAMIK}

Die wichtigsten Ergebnisse der früheren typologischen Bearbeitungen der badenzeitlichen Keramik können wir wie folgt resümieren.

J. Banner bestimmte grundlegend acht Leittypen im keramischen Bestand der „Péceler Kultur“: In den Gruppen von zweigeteilten Schüsseln, Schöpfgefäßen mit Henkel, Schöpfschalen, Fußbechern, Krügen, Schüsseln, Doppelschüsseln und Vorratsgefäßen bestimmte er insgesamt 176 Gefäßtypen und er gruppierte auch die keramischen Verzierungen. ${ }^{43}$

Auf Grund der keramischen Funde von Alsónémedi stellte J. Korek die folgenden Gefäßtypen fest: krugförmige Gefäße mit hohem Henkel, kegelförmige Gefäße mit hohem Henkel, zweiteilige Schüssel, Gefäße von Form eines Fischerbootes mit zwei Bandhenkeln, Schüsseln und Schöpfgefäße mit Griff. ${ }^{44}$

\footnotetext{
${ }^{36}$ „Als traditionell meistbeachtete Materialkategorie in der Neolithforschung ist eine entsprechende Aufbereitung und Typologisierung der keramischen Inventare des Arbeitsgebietes ein unumgänglicher Arbeitsschritt. Darauf aufbauend erfolgte eine chronologische Analyse als Beitrag zum Verständnis entsprechender Strukturen innerhalb der Standardisierungsebene Gefäßkeramik“ (FuRHOLT 2009, 203).

${ }^{37}$ BIEHL-GLESER 2003.

${ }^{38}$ „Die Zusammenstellung keramischer Varianten zu Haupt- und Untergruppen erfuhr häufig eine Interpretation als jeweils verschiedenen Kulturen zugehörig, denen eine historische Dimension
}

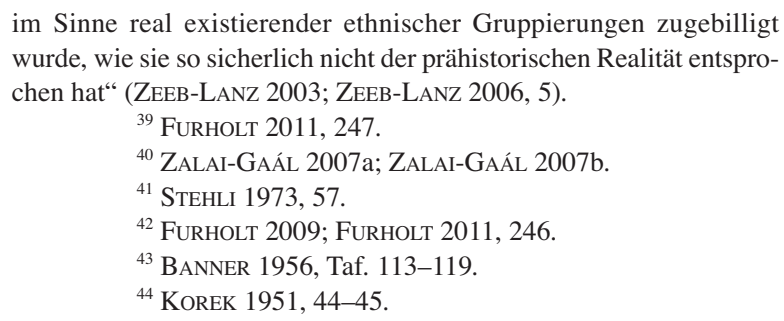
wurde, wie sie so sicherlich nicht der prähistorischen Realität entsprochen hat" (Zeeb-Lanz 2003; ZeEb-Lanz 2006, 5).

${ }^{39}$ FURHOLt 2011, 247.

${ }^{40}$ Zalai-GaÁl 2007a; ZaLai-GaÁl 2007b.

${ }^{41}$ Stehli 1973, 57.

${ }^{42}$ Furholt 2009; Furholt 2011, 246.

${ }^{43}$ BANNER 1956, Taf. 113-119.

${ }^{44}$ KoReK 1951, 44-45. 
Von Mezőcsát-Hörcsögös erwähnt N. Kalicz Fußkelche, Schöpflöffel, fischerbootförmige Gefäße und kalottenförmige Schöpfgefäße. ${ }^{45}$

Dem aktuellen Forschungsstand der Badener Kultur in Ungarn widmete M. Bondár eine Studie im Jahre $2002 .{ }^{46}$ Von der Fundstelle Balatonmagyaród-Hidvégpuszta veröffentlichte sie vier Gräber der Badener Kultur. ${ }^{47} \mathrm{Im}$ Gräberfeld von Budakalász sonderte sie grundlegend 17 keramische Typen ab (goblets, ladles, dippers, suspension vessels, mugs, pitchers, jugs, scooping vessels, handled cups, beakers, handled pots, pots, amphoras, bowls, handled bowls, bipartite bowls, clay spools, wagon model) ${ }^{48} \mathrm{Im}$ Fall des Gräberfeldes Pilismarót-Basaharc erarbeitete sie eine noch ausführlichere Gruppierung der Grabgefäße. Sie bestimmte insgesamt 21 Typen sowie 7 Typen von Amphoren, 7 Typen von „Gefäßen“ (pots), 3 Typen von Vorratsgefäßen (storage jars), 8 Typen von Schalen (mugs), 9 Typen von Töpfen (jugs), 3 Typen von Tassen (cups), je einen Typ von blumentopfförmigen Gefäßen (beakers, flowerpot-shaped vessels), von Schöpfgefäßen (scooping vessels), von Hängegefäßen (suspension vessels), Schüsseln (dish-pots), Häfen (bowls), Miniaturgefäßen (miniature vessels), von Stempeln (stamp), von Kegeln (clay cones), von Spinnwirteln (spindle whorls), von Rollern (rollers, „cylindrical or spool-like clay object“, „,colonette“), von Tonlöffeln (clay spoon), von Trinkhörnern (clay drinking horn), von Tierdarstellungen (animal depictions) und von Wagenmodellen (wagon model). ${ }^{49}$

In einer neueren Studie suchte M. Bondár auf Grund der Funde und Befunde von der Siedlung NagyútGöbölyjárás II (Westtransdanubien) die Frage nach, wie das typologische System von V. Němejcová-Pavúková verallgemeinert werden kann. ${ }^{50}$ Dabei benutzte sie 79 keramische Typen und nicht weniger als 221 Varianten..$^{51}$ Dazu berücksichtigte sie auch die früheren Periodisationssysteme der Badener Kultur von E. Neuštupný, A. Točík und V. Němejcová-Pavúková. ${ }^{52}$

Eine detaillierte keramische Typologie stammt von T. Horváth, die die keramischen Siedlungsfunde von Balatonőszöd-Temetői-dülő grundlegend in 26 Typen und innerhalb dieser Typen in mehrere Untertypen einreihte (Henkeltassen, Schöpfer, Milchtassen, Kleinkrüge, Krüge, topfartige Krüge, Amphoren, Amphoren mit Doppelhenkel, gynäkomorphe Amphoren, Hängegefäße, Flaschen, Fußbecher, Trinkschalen, Schüsseln, Töpfe, Deckel/Backteller, Spinnwirtel, Wagenradmodelle, Oval-Zylindersäule, Löffel, Maske, Idole, Stempel, Hausmodelle, anthropomorphe Gefäße mit Fuß, Rhyton). ${ }^{53}$ Die Mehrheit dieser Typen - Siedlungsfunde - sind im keramischen Befund der untersuchten Gräberfelder nicht auffindbar. T. Horváth widmete eine Studie auch der chronologischen bzw. räumlichen Lage und den interkulturellen Beziehungen der Boleráz-, Baden- und Kostolac-Kulturen. ${ }^{54}$

Die Funde und Befunde aus dem Brandgräberfeld der Badener Kultur in Malá nad Hronom (Kicsind, Slowakei) mit vier Gräbern beschrieb G. Nevizánsky. ${ }^{55}$ Er arbeitete auch ein typologisches Schema der Amphoren der Badener Kultur aus und sonderte damit 10 Serien mit Unterserien und Verzierungsstile A-N unter ihnen ab. Er stellte weiterhin fest, dass sich diese Gefäßserie vom chronologischen Aspekt aus markant in zwei, beinahe geschlossene Gruppen gliedert. ${ }^{56}$

Die Funde der in Balatonkeresztúr-Réti-dülő geborgenen 15 Bestattungen wurden in die Analyse nicht aufgenommen. Sz. Fábián, die auch die Forschungsgeschichte der Badener Kultur ausführlich behandelt, bestimmte 12 Haupttypen im keramischen Material sowie Schalen, Näpfe, Krüge, Schüsseln, amphorenförmige Gefäße, amphorenförmige Speichergefäße, Töpfe, Speichergefäße, zweigeteilte Schüsseln, Schapfe, blumentopfförmige Gefäße sowie Sonderformen.$^{57}$ Die Siedlungskeramik von Abony (Fundstelle 49) wurde dann nach folgenden Typen gruppiert: „cups, mugs and jars, pots and bowls, Bodrogkeresztúr-like pottery forms, Furchenstich decoration, Balaton-Lasinja-like forms, Lower Danube style". ${ }^{58}$

In einem Fundstellenkatalog mit 199 Fundorten und 52 ungesicherten Fundorten der Badener Kultur beschrieb und veröffentlichte jüngst C. Sachße auch die Fundumstände, Bestattungssitten, Funde sowie Pläne. ${ }^{59}$ Sie

\footnotetext{
${ }^{45}$ KALICZ 1999, 86-87.

${ }^{46}$ BONDÁr 2002.

${ }^{47}$ BONDÁR 1987.

${ }^{48}$ BONDÁR 2009a, 246-289.

${ }^{49}$ BONDÁr 2015, 163-196.

${ }^{50}$ Das typologische System von V. Němejcová-Pavúková umfasst die Phase I mit Perioden IA, IB und IC, die Phase II mit Perioden IIA, IIB und Phase III (NĚMEJCOVÁ-PAVúkovÁ 1981; NĚMEJCOVÁ-PAVÚKovÁ 1984; Bondár 2010, 312, Tab. 3).
}

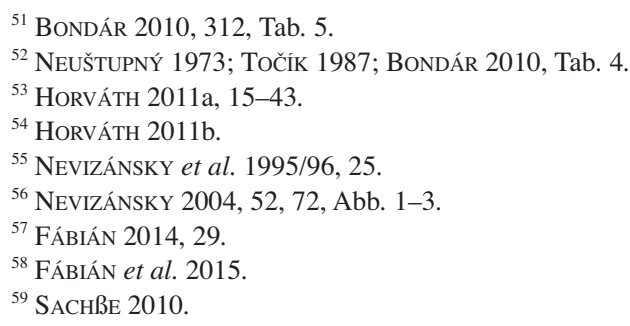

${ }^{51}$ Bondár 2010, 312, Tab. 5.

${ }^{53}$ HORVÁTH 2011a, 15-43.

${ }^{54}$ HORVÁth 2011 b.

${ }^{57}$ FÁBIÁN 2014, 29

${ }^{59}$ SACHßE 2010. 
betrachtete auch die Fragen des Übergangs zur Spätkupferzeit (Furchenstichkeramik und Protoboleráz), der frühen Badener Kultur (Boleráz) und der klassischen und späten Badener Kultur. ${ }^{60}$

Radiokarbondaten stehen von zwei Nekropolen zur Verfügung. Die relative und absolute chronologische Stellung der Gräber von Budakalász untersuchte Zs. Siklósi mit Hilfe von Seriation und Korrespondenzanalyse von AMS-Daten. Die Proben zur Gewinnung von 14C-Daten stammen aus Menschenknochen. ${ }^{61}$ Die Radionkarbondaten von Pilismarót-Basaharc wurden von D. Hamilton bestimmt; die Proben dazu stammen aus gebrannten Menschenknochen. ${ }^{62}$

Die aufgezählten Klassifikationen der prähistorischen Keramik entsprechen den Voraussetzungen einer hierarchisch aufgebauten Merkmalanalyse oder metrischen Analyse nicht: In diesen Fällen handelt es sich um Ergebnisse von früheren Forschungsmethoden.

\section{KLASSIFIKATION DER FUNDE UND BEFUNDE}

Im Laufe der formalen Gliederung der Keramik muss berücksichtigt werden, dass es keine zwei Gefäße gibt, die in allen Merkmalen gleich sind. Im besten Fall finden wir nur solche, die durch Übereinstimmung in sehr vielen Merkmalen ähnlich sind. Um die Vielzahl der Variationen überschaubar zu machen, müssen sie in Typen untergliedert werden - bestätigt auch M. Meisenheimer. ${ }^{63}$ Die zur Typenfindung verwendeten Merkmale beruhen zugleich immer auf einer subjekiven Auswahl und sind von der Fragestellung abhängig - stellt S. Hansen fest. ${ }^{64}$ Bemerkt werden muss gleichzeitig, dass die relativchronologisch gewonnenen Stufen, Phasen und Perioden nur als Hilfsmittel der Verständigung dienen können. ${ }^{65}$

Unser hierarchisch aufgebautes typologisches System besteht aus Klassen, Gattungen, Serien, metrischen Gruppen, Typen und Formvarianten. Die Definition der Klassen wurde auf die Längen-Breiten-Verhältnissen der Grabgefäße gestützt. Keramiken, deren Höhe länger ist als die größte Breite, wurden in die Klasse 1 (Hochgefäße) gruppiert. Gefäße, deren Breite größer ist als die größte Länge, bilden die Klasse 2 (Breitgefäße). Die Sonderformen von Keramiken gehören in die Klasse 3. Die Definition der Gattungen wurde auf unsere früheren Klassifikationen der lengyelzeitlichen bzw. früh- und mittelkupferzeitlichen Grabkeramiken gestützt. ${ }^{66}$ Die keramischen Serien stellten wir anhand der Unterschiede der A-Indexwerte (Längen-Breiten-Indizes) fest. Die weitere Gruppierung der untersuchten Keramiken wurde auf Grund des Verhältnisses verschiedener Gefäßteile zueinander und anhand der Gefäßformen vorgenommen: Somit konnten wir die metrischen Gruppen (mGruppen) feststellen. Innerhalb der metrischen Gruppen wurden die Typen schon allein auf Grund der Gefäßformen bestimmt. „Die Typen sind interpretierte Klassen. Die Interpretation findet hierbei in unterschiedlichen Kategorien statt. Die Bedeutung eines Typs kann funktional, chronologisch, räumlich, sozial und kulturell beziehungsweise symbolisch sein. In der Praxis ergeben sich Probleme daraus, dass in einer Typologie diese Kategorien oft nicht klar getrennt werden und meistens nicht klar trennbar sind" - stellen O. Nakoinz und M. Hinz fest. ${ }^{67}$ Wir können ihnen zustimmen. Die jeweiligen Typologien sind in einem bestimmten Gebiet für eine bestimmte Epoche gültig und sie werden innerhalb der Gemeinschaft verwendet, die sich auf dieses Gebiet und diese Zeit spezialisiert hat. ${ }^{68}$ Als letzter Schritt der Klassifikation wurden die Formvarianten auf der Basis der Unterschiede der Keramiken definiert.

Die Gefäßformmerkmale haben wir also durch eine Reihe von Messwerten kodiert, die je nach Erhaltungsgrad der Gefäße in unterschiedlicher Menge vorhanden sind. ${ }^{69}$ Gemessen wurden die Gefäßhöhe, Gefäßbreite, der Randdurchmesser, der Bodendurchmesser bzw. die Verhältnisse zwischen gewissen Gefäßteilen.

${ }^{60}$ SACHßE 2009, 155-157.

${ }^{61}$ SiKLÓsI 2009. Die 14C-Untersuchungen wurden in Wien (Vienna Environmental Research Accelerator) vorgenommen.

${ }^{62}$ HAMilton 2015, Tabl. 1 (Scottish Universities Environmental Research Centre).

${ }^{63}$ Meisenheimer 1989, 9.

${ }^{64}$ Hansen 2014, 253.

${ }^{65}$ Ebenda.

${ }^{66}$ ZALAI-GAÁl 2007a; ZALAI-GAÁL 2007b.

${ }^{67}$ NAKOINZ-HINZ 2015, 225.
${ }^{68}$ „Das Typenkonzept ist das einfachste und grundlegendste latente Modell der Archäologie. Die weiteren latenten Modelle, die besprochen werden sollen, beruhen hierauf. Es setzt jedoch voraus, dass Typen, also letztlich die Kombination von Einzelmerkmalen, mindestens ebenso signifikant sind wie die Einzelmerkmale selbst" (NAKOINZ-HINZ 2015, 228).

${ }^{69}$ Furholt 2009, 30-31. 


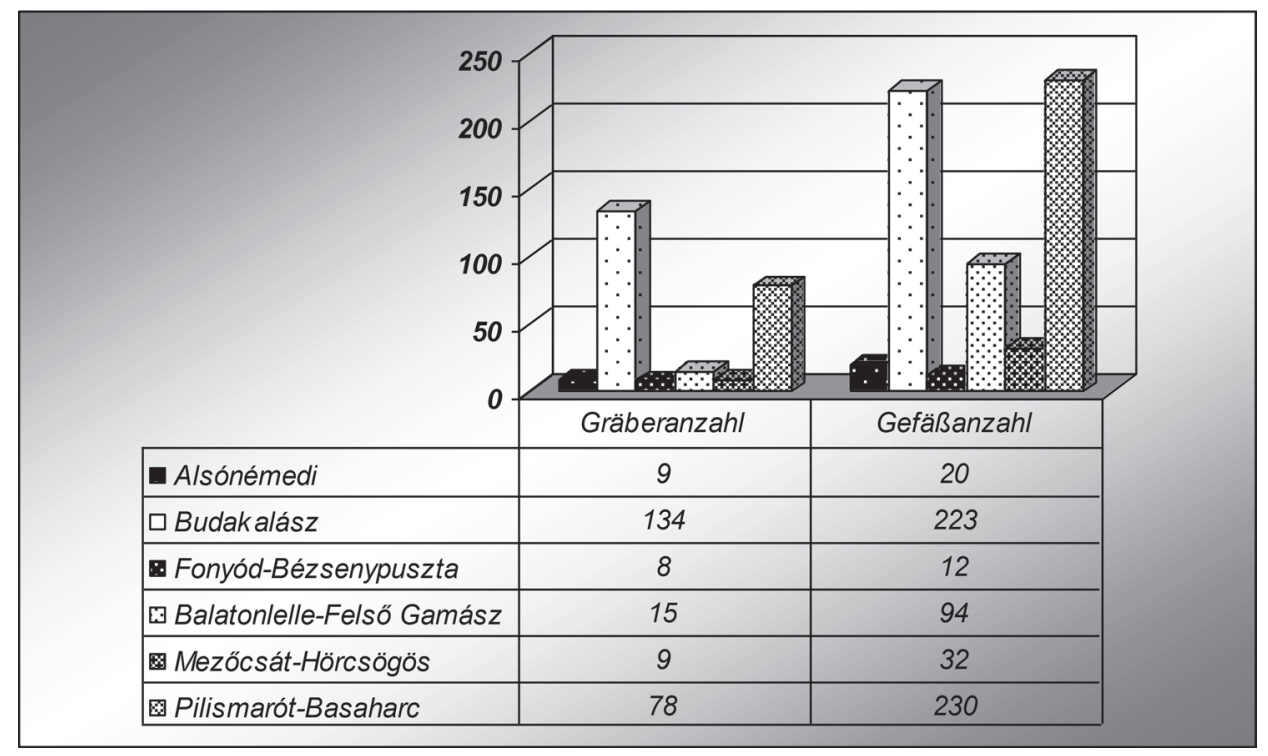

Diagramm 1. Anzahl der untersuchten Gräber und Grabgefäße

Insgesamt wurden 611 keramische Funde aus 253 Gräbern von den sechs untersuchten Nekropolen der Badener Kultur in die Analyse einbezogen. Die Anzahl der Gräber und der Keramiken in diesen Gräberfeldern zeigt Diagramm 1.

Die Struktur des in der vorliegenden Arbeit verwendeten, hierarchisch aufgebauten typologischen Systems der untersuchten Grabkeramik der Badener Kultur stellt Tabelle 1 dar.

Klasse 1: Hochgefäße mit Gattungen 1a (Fußgefäße), 1b (Henkelbecher), 1c (dreigliedrige Becher), 1d (Näpfe), 1e (Schultergefäße).

Klasse 2: Breitgefäße mit der Gattung 2a (Henkeltassen), den Gattungen 2b (zweigeteilte Schüsseln), 2c (konische Schüsseln), 2d (profilierte Schüsseln), 2e (konische Schalen), 2f (profilierte Schalen), 2g (Tonlöffel).

Klasse 3: Sonderformen mit den Gattungen 3a (,cylindrical or spool-like clay object“, „colonette“), 3b (Tierfiguren), 3c (Wagenmodelle), 3d (Tondeckel), 3e (Tonstempel), 3f (Tonhorn), 3g (Hornapplikationen), 3h (Tonaltärchen).

Die Klasse 1 der untersuchten Badener Grabkeramik vertreten Exemplare, deren Höhe größer ist als die größte Breite. Die Artefakte von den Hochgefäßen konnten gemäß fünf Gattungen gruppiert werden (Tabelle 1).

Tabelle 1. Verteilung der badenzeitlichen Grabkeramik gemäß typologischen Einheiten

\begin{tabular}{|c|c|c|c|}
\hline \multirow{2}{*}{ Keramische Gattungen } & \multicolumn{3}{|c|}{ Typologische Einheiten } \\
\hline & Serien & mGruppen & Typen \\
\hline Fußgefäße (1a) & 5 & 10 & 27 \\
\hline Henkelbecher (1b) & 7 & 22 & 83 \\
\hline Dreigliedrige Becher (1c) & 5 & 8 & 17 \\
\hline Näpfe (1d) & 2 & 4 & 13 \\
\hline Schultergefäße (1e) & 4 & 6 & 10 \\
\hline Henkeltassen (2a) & 4 & 9 & 30 \\
\hline Zweigeteilte Schüsseln (2b) & 1 & 1 & 6 \\
\hline Konische Schüsseln (2c) & 2 & 5 & 17 \\
\hline Profilierte Schüsseln (2d) & 2 & 2 & 13 \\
\hline Konische Schalen (2e) & 1 & 2 & 6 \\
\hline Profilierte Schalen (2f) & 2 & 6 & 21 \\
\hline Tonlöffel $(2 \mathrm{~g})$ & 2 & 2 & 11 \\
\hline Sonderformen $(3 \mathrm{a}-\mathrm{h})$ & 8 & 8 & 8 \\
\hline Insgesamt & 45 & 85 & 262 \\
\hline
\end{tabular}


Gattung 1a - Fußgefäße. Die Gefäße dieser Gattung können in zwei formale Teile, also eine Schüssel oder Schale und einen Hohlfuß, gegliedert werden. In die Analyse konnten wir 254 Exemplare aus 176 Bestattungen von 32 Nekropolen einbeziehen.

Die untersuchten Fußgefäße der Badener Kultur erscheinen in 5 Serien, 10 mGruppen und 27 Typen. 86,05 \% ( $\mathrm{n}=37)$ der Fußgefäßgräber stammt aus Pilismarót-Basaharc, wo 24 Gefäßtypen dieser Gattung nachgewiesen werden konnten. Nicht mehr als 9,30 \% $(\mathrm{n}=4)$ solcher Befunde ist in Balatonlelle-Felső Gamász (3 Typen) und nicht mehr als 4,65 \% ( $\mathrm{n}=2)$ in Mezőcsát (2 Typen) typisch. In den anderen Nekropolen sind Fußgefäße nicht belegt.

Die Klassifizierung der Fußgefäße nahmen wir auf Grund folgender Indizes vor. ${ }^{70}$ Die Serien wurden mit Hilfe des Indexwertes al (Höhe des Hohlfußes/Höhe der Schüssel) bestimmt. Zur Bestimmung der mGruppen verwendeten wir folgende Indizes. Die Hohlfüße können grundlegend durch Vergleich der Indexwerte Fa (Hohlfußhöhe/größte Breite des Fußes) und fb (Halsbreite/größte Breite des Fußes) gruppiert werden, während die Schüsselteile durch den Vergleich der Indexwerte Sa (Schüsselhöhe/größte Schüsselbreite) und Sb (Mündungsbreite/ Halsbreite) klassifiziert wurden. Zur Bestimmung der Varianten innerhalb der einzelnen mGruppen berücksichtigten wir in erster Linie die Profilform.

R. Gleser rechnet Keramiken, die einen Randdurchmesser von $70 \%$ bis $130 \%$ der Höhe aufweisen, zur Gattung der Becher. ${ }^{71}$ Ein weiteres Kriterium können auch die mehr oder weniger betonte Halspartie und ein HöhenBreiten-Index von ca. $100 \%$ darstellen. $^{72}$

Bei der Klassifizierung der Gattungen der Becher berücksichtigten wir folgende Indizes: Index A (größte Höhe/größte Breite), Index D1 (Bauchbreite/Bodenbreite), Index E1 (Höhe des Unterteils/Höhe des Oberteils), Index E2 (Schulterhöhe/Höhe des Unterteils) und Index E3 (Halshöhe/Bauchhöhe) ${ }^{73}$ Als der erste Schritt der Analyse der Becherformen bestimmen wir die Gattungen auf Grund der Längen-/Breitenverhältnisse der Gefäße. Darauf folgt die Bestimmung der Typengruppen anhand des Verhältnisses zwischen der Bauchbreite und der Bodenbreite. Die Varianten (Typen) stellten wir auf Grund des Indexes E1 und der Formgebung der Gefäße fest, während die Definition der Untervarianten ausschließlich auf Grund der Abweichungen der Profilierung geschah.

Gattung $1 \mathrm{~b}-$ Henkelbecher. Verschiedene Typen der Henkelbecher sind in allen untersuchten Nekropolen vorhanden. Sie treten in 7 Serien, 22 mGruppen und 83 Typen auf. Fast die Hälfte, 40,94\% $(n=70)$ der Bestattungen mit solchen Gefäßen stammt aus Budakalász (42 Typen). Der Anteil der Henkelbechergräber nimmt in Balatonlelle 28,07 \% ( $\mathrm{n}=48)$ (29 Typen), Pilismarót 16,96\% $(\mathrm{n}=29)(12$ Typen), Mezőcsát 7,02\% ( $\mathrm{n}=12)$ (9 Typen), Alsónémedi 4,09\% ( $\mathrm{n}=7$ ) (6 Typen) und Fonyód nur 2,92 \% ( $\mathrm{n}=5)$ (5 Typen) ein.

Gattung 1c - dreigliedrige Becher. Die Exemplare der Gattung 1c der dreigliedrigen Becher kommen in 5 Serien, 8 mGruppen und 17 Typen vor. Die Mehrheit, 36,36 \% $(n=12)$ der Gräber mit solchen Gefäßen wurde in Budakalász ( 9 Typen) und 24,24 \% $(n=8)$ in Pilismarót (5 Typen) nachgewiesen. 18,18\% $(n=6)$ der Befunde stammt aus Balatonlelle (6 Typen), 12,12 \% ( $\mathrm{n}=4)$ aus Mezőcsát (3 Typen) und 9,09 \% ( $\mathrm{n}=3)$ aus Alsónémedi (2 Typen). Im Gräberfeld von Fonyód ist kein Grab mit dieser keramischen Gattung zu belegen.

Gattung 1d - Näpfe. Typisch ist es bei diesen Keramiken, dass die größte Gefäßlänge zwischen 0,70 bis 0,89-mal größer ist als die größte Breite. Die Artefakte können gemäß 2 Serien, 4 mGruppen und 13 Typen gruppiert werden. Die Mehrheit, 70,83 \% ( $\mathrm{n}=17)$ der Napfgräber tritt in Pilismarót auf (10 Typen), während sie in Budakalász in $12,50 \%(\mathrm{n}=3)$ (3 Typen) erscheinen. 8,33\% $(\mathrm{n}=2)$ der Bestattungen ist in Balatonlelle bekannt (2 Typen) und in Alsónémedi und Fonyód tritt je ein solches Grab mit je einem Typ auf. Gräber mit dieser Gattung fehlen in Mezőcsát.

Gattung 1e - Schultergefäße. Die dieser Gattung zugeordneten Keramiken bilden 4 Serien, 6 mGruppen und 10 Typen. Die Hälfte, 50,0 \% ( $\mathrm{n}=7)$ der mit solchen Gefäßen ausgestatteten Bestattungen erscheint in Pilismarót (5 Typen) und 21,43\% ( $\mathrm{n}=3)$ in Fonyód (2 Typen). 14,29\% $(\mathrm{n}=2)$ der Gräber ist in Mezőcsát vorhanden (2 Typen). In Alsónémedi kennen wir eine solche Bestattung. Gräber mit der diskutierten Gattung wurden in Balatonlelle nicht nachgewiesen.

${ }^{70} \mathrm{Zu}$ den Maßbezeichnungen von Fußgefäßen: ZALAI-GAÁL 2015, Abb. 1; ZALAI-GAÁL 2016, Abb. 1; ZALAI-GAÁl et al. 2014, Abb. 2.

${ }^{71}$ GLESER 1995, 38

${ }^{72}$ Strobel 1996, 25.
${ }^{73} \mathrm{Zu}$ den Maßbezeichnungen der Becher: ZALAI-GAÁL 2015a, Abb. 2; ZALAI-GAÁL 2016, Abb. 2-3; ZALAI-GAÁL et al. 2014, Abb. 8 und 16 . 
Der Klasse 2 der untersuchten kupferzeitlichen Grabkeramik wurden Gefäße zugeordnet, deren Breite größer ist als die absolute Länge (Tabelle 1). ${ }^{74}$

Gattung 2a - Henkeltassen. Die größte Breite der Gattungen von Henkeltassen, Schüsseln und Schalen entspricht etwa der doppelten Höhe. ${ }^{75}$ „Die Schwierigkeiten bei der Klassifikation und Gliedrung der Keramik, die auf den Umstand, dass innerhalb der neolithischen Gefäße keine Uniformität gegeben ist, zurückzuführen sind, zeigten sich bei der Typisierung von Schüsseln und Schalen. “"76

In die Gattung 2a wurden niedrige Henkeltassen mit 4 Serien, 9 mGruppen und 30 Typen zugeordnet. Die Gräber mit diesen Keramiken sind besonders in Budakalász typisch, wo sie durch 71,67 \% $(n=43)$ vertreten sind (24 Typen). Ihr Anteil beträgt je 10,0 \% ( $n=6-6)$ in Pilismarót (3 Typen) und Balatonlelle (5 Typen), in Mezőcsát $5,0 \%(\mathrm{n}=3)$ (3 Typen) und in Alsónémedi 3,33\% $(\mathrm{n}=2)(2$ Typen). Diese Gattung ist in den Bestattungen von Fonyód nicht vertreten.

Gattung $2 b$ - zweigeteilte Schüsseln. Die Exemplare dieser Schüsseln erscheinen in einer Serie und einer mGruppe bzw. 6 Typen. Fast die Hälfte, 45,45 \% $(\mathrm{n}=5)$ der Gräber mit solchen Gefäßen ist in Budakalász (4 Typen), 36,36 \% $(\mathrm{n}=4)$ in Balatonlelle (3 Typen) und 18,18 \% $(\mathrm{n}=2)$ in Alsónémedi (1 Typ) zu belegen. Beachtenswert ist, dass Gräber mit zweigeteilten Schüsseln in den Nekropolen von Pilismarót, Fonyód und Mezőcsát nicht erscheinen.

Gattung 2c - konische Schüsseln. Diese Keramiken wurden in 2 Serien, 5 mGruppen und 17 Typen gruppiert. Die Mehrheit, 97,22 \% ( $\mathrm{n}=35)$ der mit solchen Keramiken versehenen Bestattungen kennzeichnet das Gräberfeld von Pilismarót-Basaharc (17 Typen). In Balatonlelle-Felső Gamász kommt nur ein solches Grab mit einem Typ vor. Diese Gattung ist in den Gräbern von Budakalász, Alsónémedi, Fonyód-Bézsenypuszta und MezőcsátHörcsögös nicht vorhanden.

Gattung 2d - profilierte Schüsseln. Die Keramiken dieser Gattung treten in 2 Serien, 2 mGruppen und 13 Typen auf. Die Mehrheit, 81,25 \% ( $\mathrm{n}=39)$ der Gräber stammt mit diesen Stücken auch in diesem Fall aus Pilismarót (12 Typen). 12,50 \% $(\mathrm{n}=6)$ und 4,17 \% $(\mathrm{n}=2)$ wurde in Budakalász und Fonyód nachgewiesen (je 2 Typen). Gräber mit Exemplaren der erörterten Gattung können in Alsónémedi und Mezöcsát nicht belegt werden.

Gattung 2e - konische Schalen. Bei den Schalen variiert das Verhältnis zwischen Höhe und größter Breite von 0,44 bis 0,70 . Sie bilden 1 Serie, 2 mGruppen und 6 Typen. Die Gräber mit solchen überwiegen; mit 77,78 \% $(\mathrm{n}=7)$ sind sie in Pilismarót charakteristisch (5 Typen) und noch je eine solche ist in Budakalász (1 Typ) und Mezőcsát (1 Typ) vorhanden. Solche Keramiken wurden in den Bestattungen von Balatonlelle, Alsónémedi und Fonyód nicht deponiert.

Gattung $2 \mathrm{f}$ - profilierte Schalen. Hierzu gehören Schalen mit profilierter Wandung in 2 Serien, 6 mGruppen und 21 Typen. Je 31,58 \% ( $\mathrm{n}=18-18)$ der Gräber mit diesen Gefäßen kennen wir von Budakalász (9 Typen) und Balatonlelle (8 Typen), während der Anteil dieser Befunde in Mezőcsát 12,28 \% ( $\mathrm{n}=7$ ) (4 Typen) einnimmt. Von Alsónémedi und Fonyód stammt je ein solches Grab.

Gattung $2 \mathrm{~g}$ - Tonlöffel. Diese Exemplare treten in 2 Serien, 2 mGruppen und 11 Typen auf. Die Mehrheit, $84,21 \%(n=16)$ solcher Bestattungen kommt in Budakalász vor (9 Typen) und nur je ein Löffelgrab ist noch in Pilismarót und Alsónémedi bekannt.

Zur Klasse der Sonderformen der untersuchten Grabkeramiken wurden 8 Serien, 8 mGruppen und 8 Typen zugestellt. Es handelt sich dabei um die Gattung 3a von ,cylindrical or spool-like clay object“, Gattung 3b von Tierfiguren, Gattung 3c von Wagenmodellen, Gattung 3d von Tondeckeln, Gattung $3 \mathrm{e}$ von Tonstempeln, Gattung $3 \mathrm{f}$ von Tonhörnern, Gattung $3 \mathrm{~g}$ von Hornapplikationen und Gattung 3h von Tonaltärchen.

Tabelle 2 zeigt zusammenfassend das Vorkommen der Gräber in den untersuchten Nekropolen gemäß keramischen Gattungen.

\footnotetext{
${ }^{74} \mathrm{Zu}$ den Maßbezeichnungen von Tassen, Schüsseln und Schalen: ZALAI-GAÁL 2015a, Abb. 4-5; ZALAI-GAÁL 2016, Abb. 4-5; ZALAI-GAÁl et al. 2014, Abb. 32.
}

\footnotetext{
${ }^{75}$ GLeSER 1995, 196; STROBel 1996, 21.

${ }^{76}$ Strobel 1996, 22; Zalai-GaÁl 2007a, 59.
} 
Tabelle 2. Vorkommen der keramischen Gattungen gemäß Gräberanzahl in den untersuchten Nekropolen

\begin{tabular}{|c|c|c|c|c|c|c|c|c|c|c|c|c|c|}
\hline \multirow{2}{*}{ Gräberfelder } & \multicolumn{13}{|c|}{ Gattungen der Grabkeramik } \\
\hline & $1 \mathbf{a}$ & $\mathbf{1 b}$ & $1 \mathrm{c}$ & $1 d$ & 1e & $2 \mathbf{a}$ & $2 \mathbf{b}$ & $2 \mathrm{c}$ & $2 d$ & $2 \mathbf{e}$ & $2 f$ & $2 \mathrm{~g}$ & $3 a-h$ \\
\hline Alsónémedi & - & 6 & 2 & 1 & 1 & 2 & 1 & - & - & - & 1 & 1 & - \\
\hline Balatonlelle-Felső Gamász & 3 & 29 & 6 & 2 & - & 5 & 3 & 1 & 1 & - & 8 & - & - \\
\hline Budakalász & 24 & 42 & - & 3 & 1 & 24 & 4 & - & 2 & 1 & 9 & 9 & 1 \\
\hline Fonyód-Bézsenypuszta & - & 5 & - & - & 2 & - & - & - & 2 & - & 1 & - & - \\
\hline Mezőcsát-Hörcsögös & 2 & 9 & 3 & 1 & 2 & 3 & - & - & - & 1 & 4 & - & - \\
\hline Pilismarót-Basaharc & - & 12 & 5 & 10 & 5 & 3 & - & 17 & 12 & 5 & 6 & 1 & 7 \\
\hline
\end{tabular}

\section{ERGEBNISSE DER METRISCHEN ANALYSE UND SERIATION}

W. M. F. Petrie war der Erste, der eine Abfolge aus dem gemeinsamen Vorkommen der Typen erarbeitet hatte. ${ }^{77}$ „Dieser Ansatz wird als Seriation bezeichnet und besteht darin, die Einträge in einer Kombinationstabelle mit geschlossenen Funden und Typen so zu sortieren, dass sich eine optimale Diagonalform ergibt. Hierbei wird eine zeitliche Entwicklung, nicht jedoch ein Evolutionsprozess, vorausgesetzt. Peter Ihm schlug vor, die Korrespondenzanalyse als robuste Schätzung der Seriation zu verwenden.“78 Eine auf die Seriation gebaute analytische Methode von prähistorischer Gefäßkeramik stammt auch von P. Stehli und A. Zimmermann. ${ }^{79}$

Man kann zugleich aber auch S. Hansen zustimmen, dass „die in die Seriation bzw. Korrespondenzanalyse einbezogenen Merkmale können, müssen aber keineswegs notwendig zeitspezifisch sein, sondern können auch Rang, Alter oder Geschlecht widerspiegeln ". ${ }^{80} \mathrm{Im}$ Fall der vorliegenden Arbeit handelt es sich aber nur um einen Versuch, die relative Chronologie der untersuchten Gräberfelder feststellen zu können. Diesen Prozess nahmen wir einerseits auf der Basis des Verhältnisses zwischen Gräbern und metrischen Gruppen der Keramik, andererseits auf Grund der Zusammenhänge zwischen Gräbern und Typen vor.

Auch O. Nakoinz und M. Hinz bestätigen: Obwohl absolute Datierungen heute zunehmend an Bedeutung gewinnen, spielt „die Typochronologie in der Archäologie noch eine wichtige Rolle und wird diese auch in den kommenden Jahrzehnten nicht verlieren“. ${ }^{81}$

\subsection{Belegungsphasen durch metrische Gruppen}

Gemäß 65 mGruppen seriierte 119 Bestattungen wurden durch das Seriationsprogramm in drei Belegungsphasen eingeteilt ( $A b b$. 2). Das Vorkommen der von diesem Gesichtspunkt aus bzw. gemäß den Typen gruppierten Gräber in den behandelten Nekropolen zeigt Diagramm 2.

Die Ergebnisse der Hauptkoordinatenanalyse der Bestattungen durch drei künstliche Phasen (Anfangsgräber, Übergangsgräber und späte Gräber) illustrieren Abbildung 2 und Tabelle 3.

Belegungsphase 1 durch mGruppen. 36,13\% $(\mathrm{n}=43)$ der gemäß mGruppen seriierten Befunde vertritt diese erste „künstliche Phase“ (Grab fg8 von Balatonlelle-Felső Gamász, pb336, pb338, pb340, pb343, pb344, pb351, pb352, pb355, pb356, pb358, pb359, pb362, pb364, pb382, pb384, pb385, pb386, pb388, pb390, pb393, pb399, pb400, pb401, pb403, pb406, pb413, pb414, pb419, pb422, pb426, pb427, pb429, pb434, pb436, pb439, pb441, pb442, pb443, pb444, pb447 und pb448 von Pilismarót-Basaharc, bk306 von Budakalász). Die Mehrheit, 91,11 \% ( $\mathrm{n}=41)$ dieser Bestattungen stammt also aus Pilismarót-Basaharc. Das Grab fg8 kam in Balatonlelle-Felső Gamász und das Grab bk306 in Budakalász zum Vorschein. Diese Befunde erscheinen links von der Achse F1 des Histogramms grün (Abb. 2, Tab. 3).

\footnotetext{
${ }^{77}$ PETRIE 1899.

${ }^{78}$ IHM 1983; NAKOINZ-HINZ 2015, 231.

${ }^{79}$ Stehli 1973; Stehli-Zimmermann 1980.
}

\footnotetext{
${ }^{80}$ HANSEN 2014, 253.

${ }^{81}$ NAKOINZ-HinZ 2015, 231.
} 


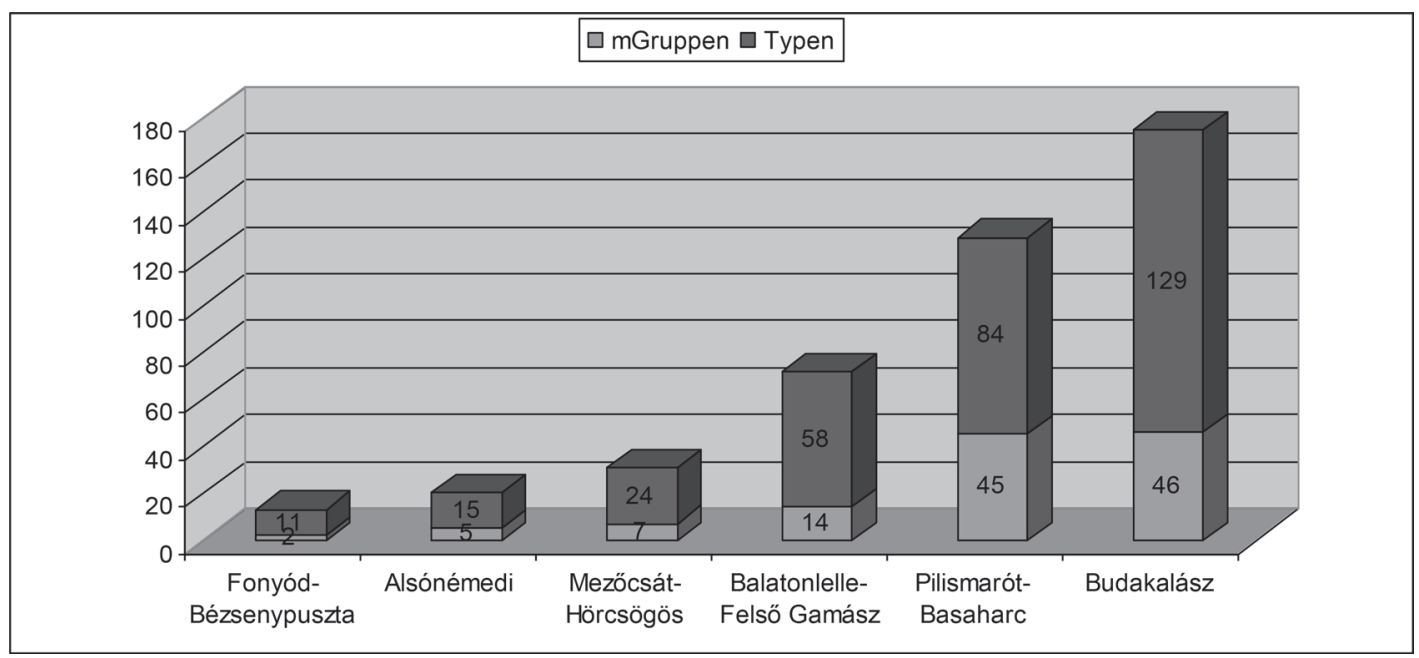

Diagramm 2. Vorkommen der mGruppen und der Typen in den untersuchten Nekropolen

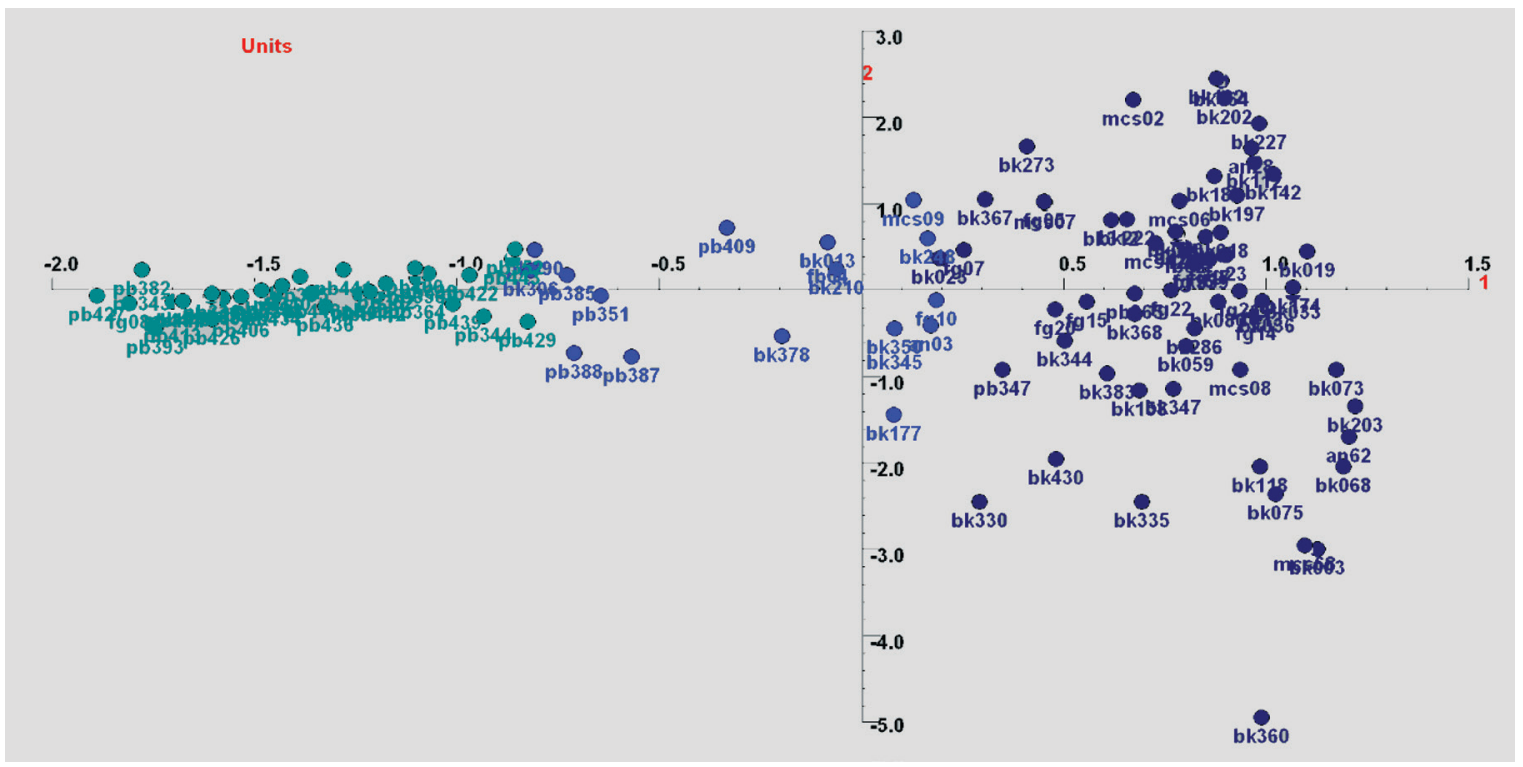

Abb. 2. Ergebnisse der Korrespondenzanalyse der gemäß mGruppen untersuchten Gräber nach drei Belegungsphasen

Belegungsphase 2 durch mGruppen. Nicht mehr als 5,88 \% $(\mathrm{n}=7)$ der Gräber kann hier zugeteilt werden (Grab bk13, bk177, bk210 und bk378 von Budakalász, fb4 von Fonyód-Bézsenypuszta, pb387 und pb409 von Pilismarót-Basaharc). Im Histogramm wird auf diese Gräber links von der Achse F1 hellblau hingedeutet (Abb. 2, Tab. 3).

Belegungsphase 3 durch mGruppen. Mehr als die Hälfte, 57,98 \% (n=69) der seriierten Befunde datiert in diese Zeit. 41 Gräber wurden in Budakalász, 13 in Balatonlelle-Felső Gamász, 7 in Mezőcsát-Hörcsögös, 5 in Alsónémedi und 1 in Fonyód-Bézsenypuszta geborgen. Zwei solche Bestattungen stammen auch aus PilismarótBasaharc (Grab an3, an4, an22, an28 und an62 aus Alsónémedi, bk3, bk18, bk19, bk23, bk33, bk36, bk59, bk68, bk70, bk73, bk75, bk80, bk112, bk118, bk132, bk142, bk158, bk164, bk174, bk189, bk197, bk202, bk203, bk222, bk227, bk248, bk273, bk286, bk312, bk330, bk335, bk339, bk344, bk345, bk347, bk350, bk360, bk367, bk368, bk383 und bk430 aus Budakalász, fb2 aus Fonyód-Bézsenypuszta, fg5, fg6, fg7, fg10, fg13, fg 14, fg15, fg16, fg17, fg20, fg21, fg22 und fg23 aus Balatonlelle-Felső Gamász, mcs2, mcs6, mcs7, mcs8, mcs9, mcs12 und mcs68 aus Mezőcsát-Hörcsögös, pb347 und pb365 aus Pilismarót-Basaharc). Diese Gräber erscheinen rechts von der Achse F1 des Historgramms dunkelblau (Abb. 2, Tab. 3). 
Tabelle 3. Vorkommen der durch mGruppen definierten Gräber nach Belegungsphasen und Nekropolen

\begin{tabular}{|c|c|c|c|}
\hline \multirow{2}{*}{ Gräberfelder } & \multicolumn{3}{|c|}{ Belegungsphasen gemäß mGruppen } \\
\hline & Phase 1 & Phase 2 & Phase 3 \\
\hline Pilismarót-Basaharc & $\begin{array}{c}41 \\
(95,35 \%)\end{array}$ & $\begin{array}{c}2 \\
(28,57 \%)\end{array}$ & $\begin{array}{c}2 \\
(2,91 \%)\end{array}$ \\
\hline Budakalász & $\begin{array}{c}1 \\
(2,33 \%)\end{array}$ & $\begin{array}{c}4 \\
(57,14 \%)\end{array}$ & $\begin{array}{c}41 \\
(59,42 \%)\end{array}$ \\
\hline Balatonlelle-Felső Gamász & $\begin{array}{c}1 \\
(2,33 \%)\end{array}$ & - & $\begin{array}{c}13 \\
(18,84 \%) \\
\end{array}$ \\
\hline Fonyód-Bézsenypuszta & - & $\begin{array}{c}1 \\
(14,29 \%)\end{array}$ & $\begin{array}{c}1 \\
(1,45 \%)\end{array}$ \\
\hline Mezőcsát-Hörcsögös & - & - & $\begin{array}{c}7 \\
(10,14 \%)\end{array}$ \\
\hline Alsónémedi & - & - & $\begin{array}{c}5 \\
(7,25 \%)\end{array}$ \\
\hline Insgesamt & 43 & 7 & 69 \\
\hline
\end{tabular}

\subsection{Belegungsphasen durch Typen}

Die Verteilung der gemäß keramischen Typen seriierten 321 Gräber in den untersuchten Nekropolen zeigen Seriationstabelle 1 und Abbildung 3.

Die durch die Typen durchgeführte Analyse macht eine engere Gruppierung der Befunde möglich. Die auf diese Weise gemachte Seriation ergab die Gruppierung von 73 Gräbern im Verhältnis zu 87 Typen (Abb. 3).

Auch bei den in der vorliegenden Arbeit vorgenommenen Korrespondenzanalysen ist eine annähernd parabelförmige Struktur zu sehen, jedoch auch eine diese Struktur überlagernde bzw. störende Clusterung der Daten. Auch M. Furholt deutete bereits an, dass „offenbar räumliche und zeitliche Faktoren bei der Anordnung der Daten eine Rolle spielen, aber sich auch bestimmte kulturell bedingte Gruppen als Cluster bzw. Keramikstile abzeichnen“. ${ }^{82}$ In diesem Fall sind grundlegend drei Belegungsphasen (künstliche Phasen) feststellbar (Abb. 3, Tab. 4).

Belegungsphase 1 durch Typen. 41,10 \% $(\mathrm{n}=30)$ der Gräber kann dieser Phase zugeordnet werden. Mit der Ausnahme des Grabs fg8 von Balatonlelle-Felső Gamász stammen alle anderen Befunde dieser Belegungsphase aus Pilismarót-Basaharc (Grab pb336, pb340, pb343, pb344, pb351, pb352, pb355, pb358, pb359, pb364, pb382, pb384, pb385, pb386, pb388, pb400, pb413, pb414, pb419, pb422, pb426, pb427, pb434, pb436, pb439, pb441, pb443, pb444, pb449). Diese Befunde erscheinen links von der Achse F1 im Histogramm (Abb. 3, Tab. 4).

29 seriierte Gefäßtypen treten in den Befunden der diskutierten Belegungsphase auf (Typengruppe 1: 1b1a3, 1b1b1, 1b1b2, 1b2b1, 1b2b3, 1b2b4, 1b3b1, 1c1a1, 1d1b2, 1d1b4, 1d1c1, 2a4b1, 2a4b2, 2c1c1, 2c1d4, 2c1d7, 2c1d8, 2c2a1, 2d1a1, 2d1a2, 2d2a10, 2d2a5, 2d2a7, 2d2a9, 2e1b1, 2e1b2, 2f1c1, 2f1c3, 3b1a1).

Belegungsphase 2 durch Typen. Nicht mehr als 2,74 \% $(n=2)$ der Gräber dürfte in eine mögliche Übergangsperiode datiert werden. All diese Befunde stammen aus Pilismarót-Basaharc (Grab pb338, pb401). Sie kommen im linken Sektor des Histogramms vor.

Nur 7 seriierte Gefäßtypen sind während dieser Zeit kennzeichnend (Typengruppen 2-4: 1b2b2, 1c2c1, 1c3a2, 2c1d5, 2d2a1, 2d2a3, 3a1a1). Die Gräber der Belegungsphase 1 und der Belegungsphase 2 erscheinen im linken Sektor des Histogramms.

Belegungsphase 3 durch Typen. Mehr als die Hälfte, 56,16 \% $(n=41)$ der Bestattungen repräsentiert die letzte Belegunsgphase. 26,83\% $(\mathrm{n}=11)$ dieser Bestattungen stammt aus Balatonlelle-Felső Gamász, 14,63 \% $(\mathrm{n}=6)$ aus Mezőcsát-Hörcsögös und mehr als die Hälfte, 48,78 \% $(\mathrm{n}=20)$ aus Budakalász. Je ein solches Grab kann in Alsónémedi und zwei andere können in Pilismarót-Basaharc belegt werden (Grab an22 von Alsónémedi, bk59, bk68, bk75, bk80, bk142, bk189, bk197, bk210, bk222, bk248, bk273, bk312, bk330, bk339, bk345, bk347, bk350, bk367, bk378 und bk383 von Budakalász, fb4 von Fonyód-Bézsenypuszta, fg5, fg6, fg7, fg13, fg15, fg16, fg17, fg20, fg21, fg22 und fg23 von Balatonlelle-Felső Gamász, mcs2, mcs6, mcs7, mcs8, mcs9 und mcs12 von MezőcsátHörcsögös, pb347 und pb390 von Pilismarót-Basaharc). All diese Bestattungen sind im rechten Sektor des Histogramms grün zu sehen (Abb. 3, Tab.4).

${ }^{82}$ Furholt 2009, 68. 
Input Correlation: 0.2291 Output Correlation: $0.9941 \%$ Variance: 3.6142

12311122222112223222121122212122312212111211211112111121222121121221211112121221221212 bbcbbbbabbabbaaalaaabbbbaaabbbaabbaabbbbbabbbbbbba aabobbbbbbbbbbbbbbbbbbbbabbbobbbbbbab 3212221212111222122111232121122212224253425515346242551622151312322614341261311412411 beaaefdedebgfdaaaedbbdef ebecdceeaedbbcee ffgfeabccbag faecgcf cgccgbbccdfdccdbefbchfecb 2212112841311781144226319414111212513461114124143631213121425323113237215112633332321 21

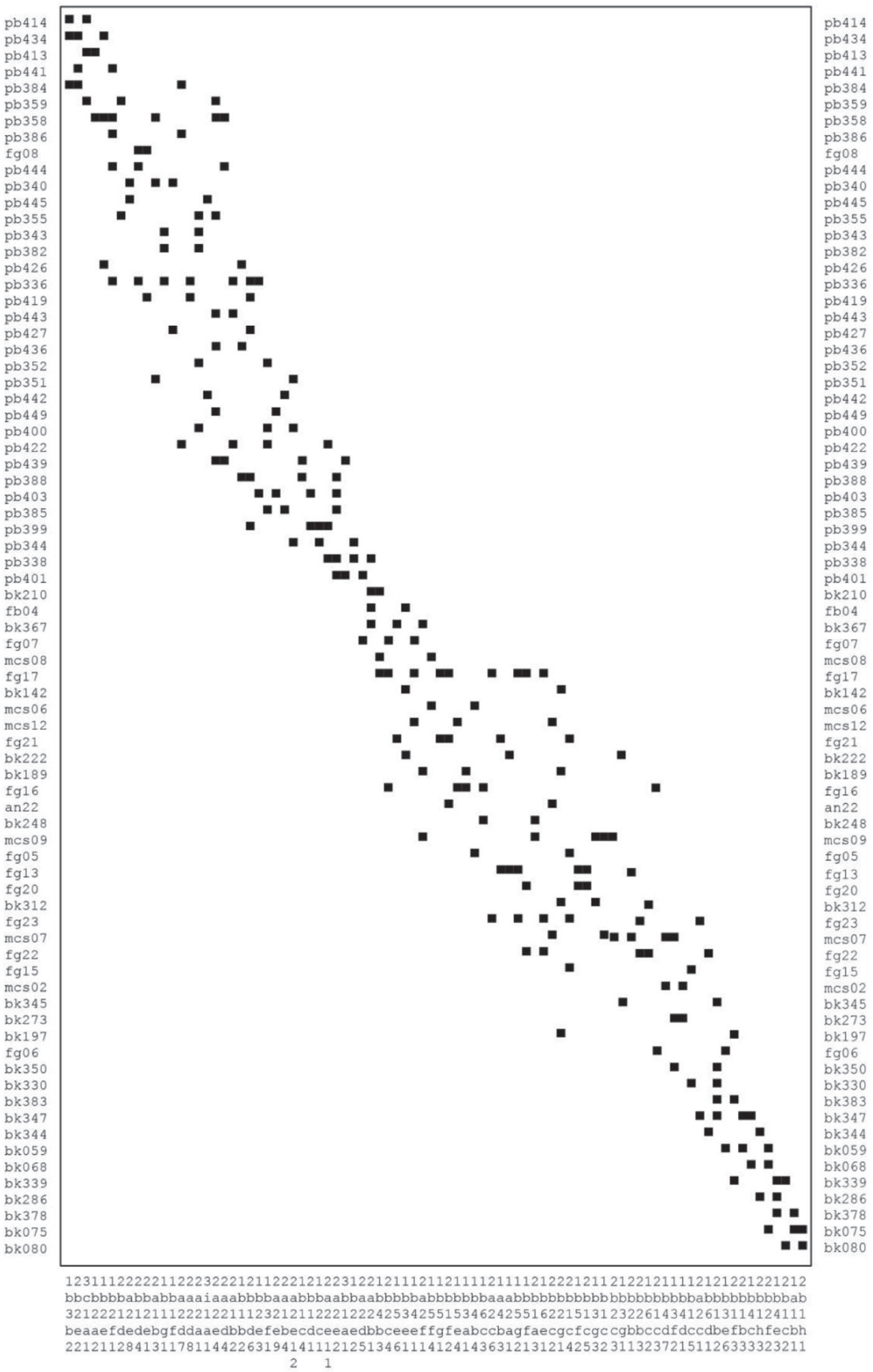

Seriationstabelle 1. Verteilung der durch Typen seriierten Gräber 
Die Mehrheit, 39 der seriierten Gefäßtypen kann zu dieser Zeit nachgewiesen werden (Typengruppe 5: 1a1b1, 1a2a1, 1b1a4, 1b3a3, 1b3a8, 1b3b2, 1b3b4, 1b3b6, 1b4a3, 1b4c1, 1b4c3, 1b5b4, 1b5b6, 1b5c1, 1b5d1, 1b5d2, 1b6b1, 1b6b2, 1b6c1, 1b6c2, 1c1b2, 1c3a4, 1d1a3, 2a2a1, 2a3b3, 2a3d1, 2a4c2, 2a4d1, 2b1a1, 2b1a6, 2e1a3, 2f1a2, 2f1a3, 2f1b2, 2f1b3, 2f1b4, 2f2b3, 2f2c3, 2f2c4). Die Befunde der Belegungsphase 3 gruppieren sich im rechten Sektor des Histogramms grün (Abb. 3, Tab. 4).

\subsection{Verbreitung der durch mGruppen und Typen seriierten Gräber gemäß Belegungsphasen}

In Pilismarót-Basaharc konnten 45 Bestattungen im Verhältnis zu den mGruppen zugeordnet werden. $91,11 \%(\mathrm{n}=41)$ von ihnen dürfte die Belegungsphase 1, 4,44\% $(\mathrm{n}=2)$ die Belegungsphase 2 und ebenso 4,44\% $(n=2)$ die Belegungsphase 3 repräsentieren. 33 Gräber wurden durch Typen zeitlich gruppiert. 87,88\% $(n=29)$ von ihnen gehört der Belegungsphase 1 und je 6,06 \% $(n=2-2)$ der Belegungsphase 2 und der Belegungsphase 3 an.

Tabelle 4. Vorkommen der durch Typen definierten Gräber nach Belegungsphase und Nekropole

\begin{tabular}{|c|c|c|c|}
\hline \multirow{2}{*}{ Gräberfelder } & \multicolumn{3}{|c|}{ Belegungsphasen gemäß Typen } \\
\hline & Phase 1 & Phase 2 & Phase 3 \\
\hline Pilismarót-Basaharc & $\begin{array}{c}29 \\
(96,67 \%)\end{array}$ & 2 & $\begin{array}{c}2 \\
(4,88 \%)\end{array}$ \\
\hline Balatonlelle-Felső Gamász & $\begin{array}{c}1 \\
(3,33 \%) \\
\end{array}$ & - & $\begin{array}{c}11 \\
(26,83 \%)\end{array}$ \\
\hline Budakalász & - & - & $\begin{array}{c}20 \\
(48,78 \%)\end{array}$ \\
\hline Mezőcsát-Hörcsögös & - & - & $\begin{array}{c}6 \\
(14,63 \%)\end{array}$ \\
\hline Alsónémedi & - & - & $\begin{array}{c}1 \\
(2,44 \%)\end{array}$ \\
\hline Fonyód-Bézsenypuszta & - & - & $\begin{array}{c}1 \\
(2,44 \%)\end{array}$ \\
\hline Insgesamt & 30 & 2 & 41 \\
\hline
\end{tabular}

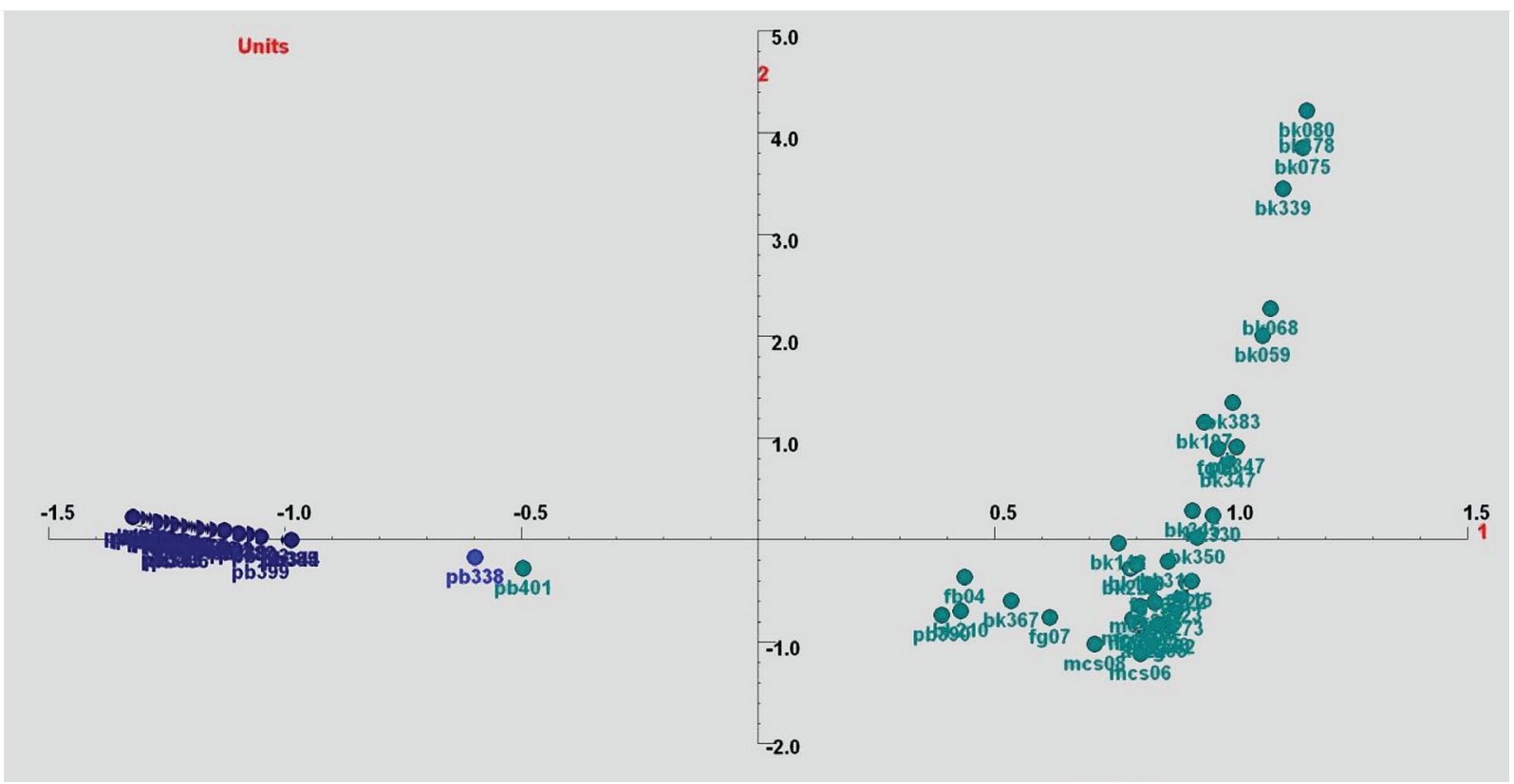

Abb. 3. Ergebnisse der Korrespondenzanalyse der durch Typen geordneten Gräber nach drei Belegungsphasen 
In der Nekropole von Budakalász sind 46 Gräber vorhanden, die gemäß Belegungsphasen in die mGruppen zeitlich eingeordnet wurden. Nur 2,17\% $(n=1)$ von ihnen gehört der Belegungsphase 1 und 8,70\% $(n=4)$ der Belegungsphase 2 an. Die Mehrheit, 89,13\% ( $\mathrm{n}=41)$ stammt aus der Zeit der Belegungsphase 3. Die zeitliche Stellung gemäß Typen ist bei 20 Bestattungen nachweisbar: Alle vertreten die Belegungsphase 3 .

Die durch die mGruppen vorgenommene Seriation ergab in Balatonlelle-Felső Gamász das Ergebnis, dass nur 7,14 \% ( $\mathrm{n}=1)$ der Gräber dieser Fundstelle die Belegungsphase 1 vertreten dürfte, während 92,86 \% ( $\mathrm{n}=13)$ schon die Belegungsphase 3 repräsentiert. Die Seriation der Gräber durch Typen weist hier darauf hin, dass ein Grab aus der Zeit der Belegungsphase 1, aber 91,67\% ( $n=13)$ aus der Zeit der Belegungsphase 3 gestammt haben dürften.

In Fonyód-Bézsenypuszta datiert eine Bestattung durch mGruppen in die Belegungsphase 2 und eine andere in die Belegungsphase 3. Gemäß Typen kann hier nur ein Grab zeitlich eingeordnet werden: Es handelt sich dabei um einen Befund der Belegungsphase 3.

In Alsónémedi findet man fünf Bestattungen, die durch mGruppen der Belegungsphase 3 zugeordnet werden können.

Auf Grund der Ergebnisse der Seriation durch mGruppen in Mezőcsát-Hörcsögös datieren sieben Bestattungen in die Belegungsphase 3. Die Untersuchung durch Typen ergab ein ähnliches Ergebnis im Fall von sechs Befunden.

\subsection{Belegungsperioden in den Gräberfeldern der badenzeitlichen Nekropolen}

Als Ergebnis der Untersuchungen durch das Unicet Programm definierte M. Furholt jüngst vier Zeitscheiben für die Badener Kultur: Zeitscheibe 1 (3650-3500 BC), Zeitscheibe 2 (3500-3350 BC), Zeitscheibe 3 (33503100 BC) und Zeitscheibe 4 (3100-2900 BC). In seiner Arbeit aus 2011 befasst er sich nur mit der Chronologie der Zeitscheiben 2 und $3{ }^{83}$ Er bestimmt weiterhin zwei Untergruppen des Netzwerks, die „nur schwach miteinander verbunden sind“: 1 . die Regionen Ungarns, der Südwest- und Westslowakei und 2. eine Untergruppe, „die sich von Wien über den gesamten mährischen Raum, über Oberschlesien bis nach Kleinpolen erstreckt“ und „,mit den südund mitteldeutschen Regionen sowie Böhmen zeichnet sich eine weitere Untergruppe ab“. ${ }^{84}$ Die Boleráz-Keramik, die die früheste Ausprägung der Badener Keramikstile darstellt, wird in diesem Kontext als ein überregionales Phänomen bestimmt. ${ }^{85}$

Mit Hilfe der vorgeführten Forschungsmethoden studierten wir die untersuchten Gräber gemäß mGruppen und auch gemäß Typen in 15 Perioden eingeordnet (Abb.4).

\subsubsection{Belegungsperioden gemäß Seriation der Gräber durch mGruppen}

Das Seriationsprogramm (Winbasp) ermöglicht, die Befunde nach höchstens 15 typochronologischen Einheiten zu gruppieren. Ein zusammenfassendes Bild über das Vorkommen der Bestattungen gemäß mGruppen in den einzelnen Perioden und den Gräberfeldern stellen Abbildung 4 und Tabelle 5 dar.

Auf die Zusammenhänge zwischen den anhand der mGruppen definierten Einheiten bei 107 Gräbern von Pilismarót-Basaharc und Budakalász und den bekannten Radiokarbondaten wird in Tabelle 6 hingedeutet.

\subsubsection{Belegungsperioden durch Typen}

Auf Grund der Seriation durch Typen kann man zwar wenigere Gräber analysieren, können zugleich engere, annehmlich relativchronologische Beziehungen durch die Anwendung dieser Methode zwischen den Bestattungen und den Nekropolen beobachtet werden. Bei diesem Versuch handelt es sich um 76 Bestattungen. Ihre Verteilung illustriert $A b b .5$.

\footnotetext{
${ }^{83}$ FURHOLT 2011, 254.

${ }^{84}$ Ebenda, 255.
}

${ }^{85}$ Ebenda, 245. 


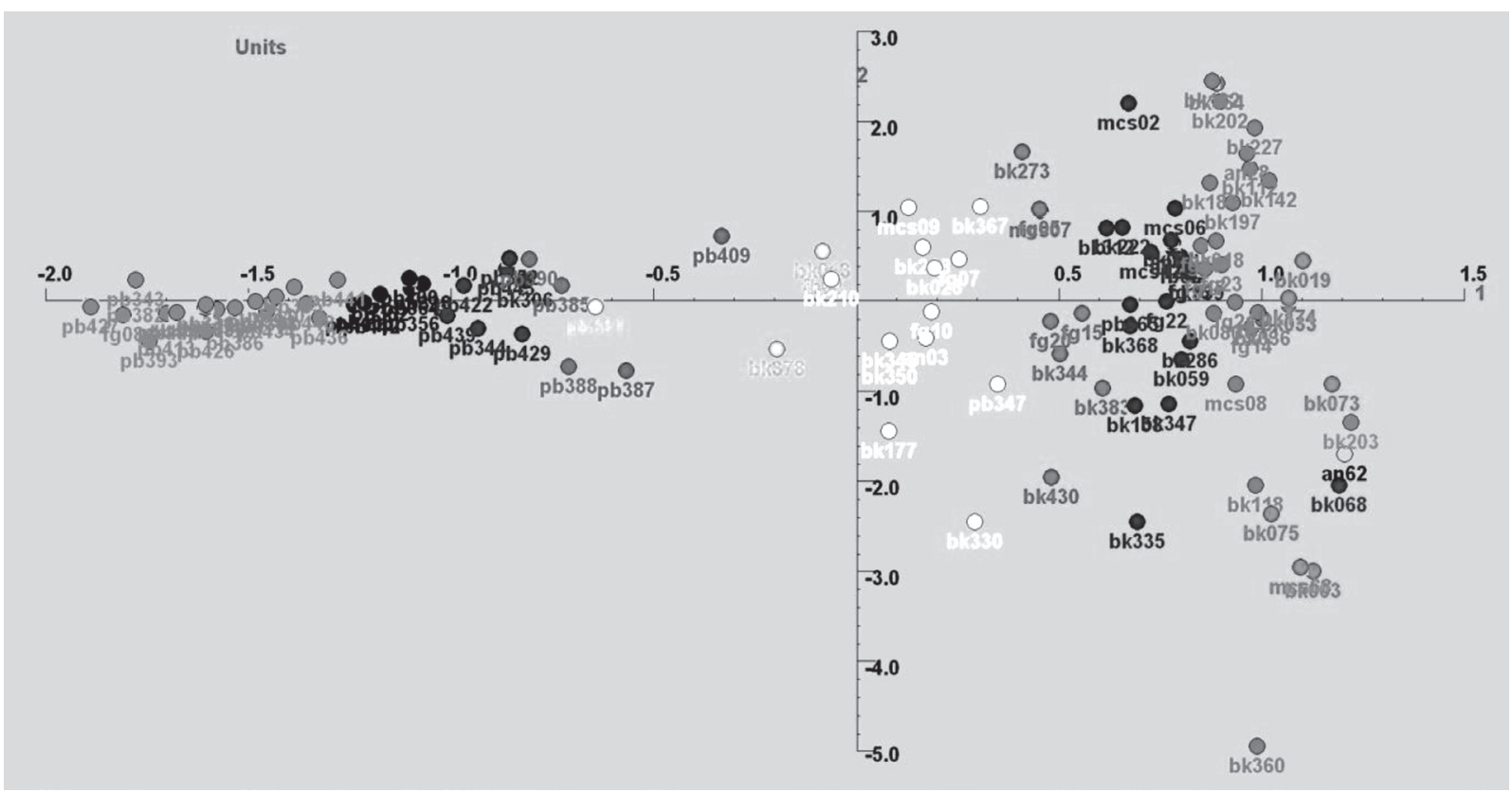

Abb. 4. Ergebnisse der Korrespondenzanalyse der gemäß mGruppen zugeordneten Gräber im Verhältnis zu 15 künstlichen Perioden

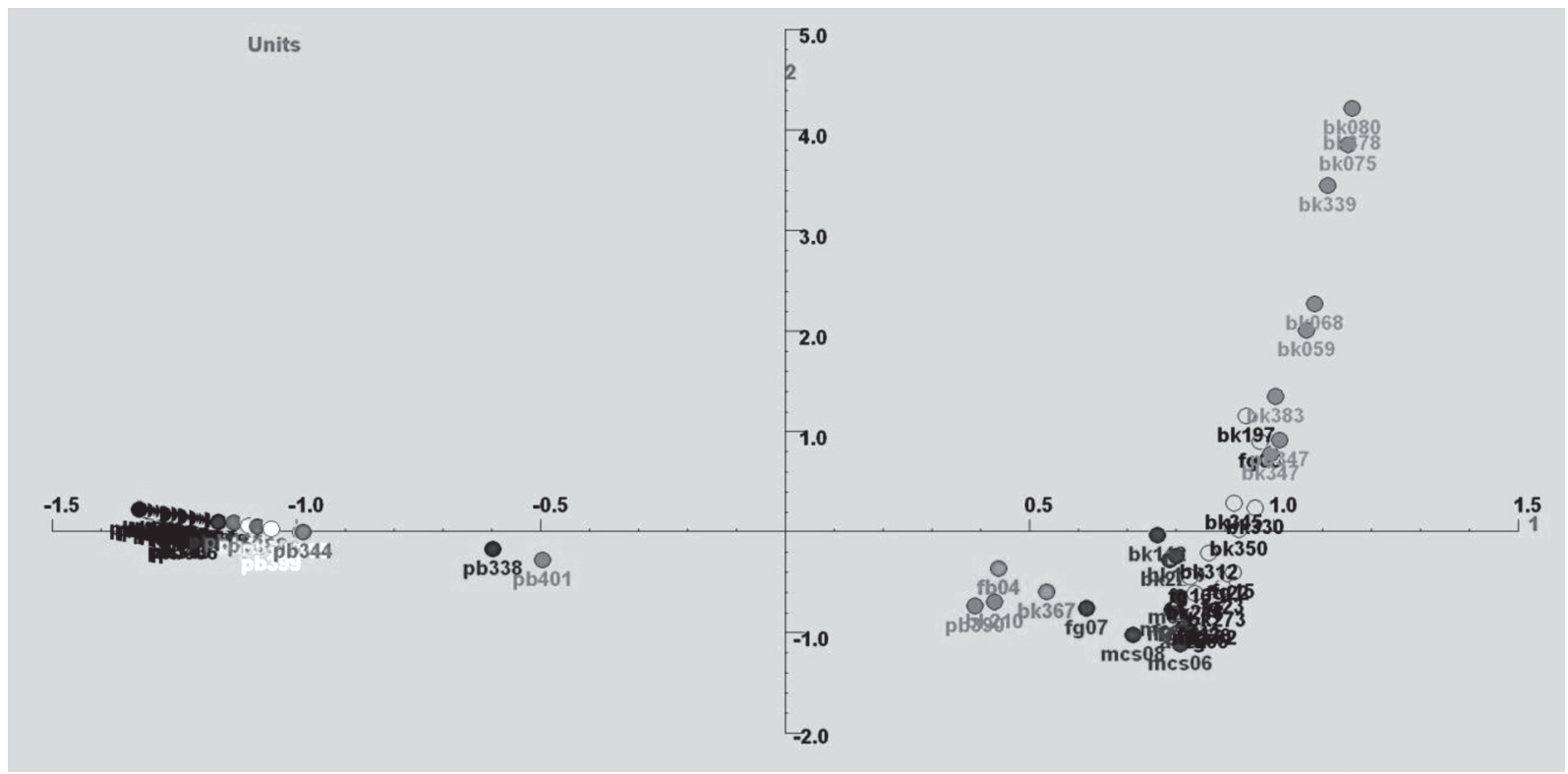

Abb. 5. Ergebnisse der Korrespondenzanalyse der gemäß Typen untersuchten Gräber nach 15 Perioden

Das Vorkommen der durch Typen zugeordneten Bestattungen gemäß Perioden zeigen Tabelle 7 und Diagramm 3.

Auf die Zusammenhänge zwischen diesen künstlich ausgezeigten Perioden und den Radiokarbondaten wird in Tabelle 8 hingedeutet.

In diesem Sinne können wir die Belegungsgeschichte der untersuchten Nekropolen im klein- und großregionalen Kontext folgenderweise resümieren. 
Tabelle 5. Vorkommen der gemäß mGruppen seriierten Gräber in den untersuchten Nekropolen

\begin{tabular}{|c|c|c|c|c|c|c|}
\hline $\begin{array}{l}\text { Perioden } \\
\text { (mGruppen) }\end{array}$ & Pilismarót & Balatonlelle & Fonyód & Budakalász & Mezőcsát & Alsónémedi \\
\hline 1. & $21(95,45 \%)$ & $1(4,55 \%)$ & - & - & - & - \\
\hline $2-6$. & 19 & - & - & - & - & - \\
\hline 7. & - & - & $1(33,33 \%)$ & $2(66,67 \%)$ & - & - \\
\hline 8. & - & $1(14,29 \%)$ & - & $4(57,14 \%)$ & $1(14,29 \%)$ & $1(14,29 \%)$ \\
\hline 9. & $1(25,0 \%)$ & $1(25,0 \%)$ & - & $2(50,0 \%)$ & - & - \\
\hline 10. & - & $3(37,50 \%)$ & - & $4(50,0 \%)$ & $1(12,50 \%)$ & - \\
\hline 11. & $1(6,67 \%)$ & $2(13,33 \%)$ & $1(6,67 \%)$ & $8(53,33 \%)$ & $2(13,33 \%)$ & $1(6,67 \%)$ \\
\hline 12. & - & $6(28,57 \%)$ & - & $13(61,90 \%)$ & $1(4,76 \%)$ & $1(4,76 \%)$ \\
\hline $13-15$. & - & - & - & $6(75,0 \%)$ & $1(12,50 \%)$ & $1(12,50 \%)$ \\
\hline Insgesamt & 42 & 14 & 2 & 39 & 6 & 4 \\
\hline
\end{tabular}

Tabelle 6. Belegungsphasen im Verhältnis zu den Radiokarbondaten

\begin{tabular}{|c|c|c|}
\hline $\begin{array}{c}\text { Zeiträume } \\
\text { gemäß mGruppen }\end{array}$ & $\begin{array}{c}\text { Radiokarbondaten } \\
\text { (cal BC) }\end{array}$ & $\begin{array}{c}\text { Zeitscheiben } \\
\text { nach FURHOLT }(2009,2011)\end{array}$ \\
\hline \multirow{6}{*}{ Zeitraum 1} & $3500-3350$ (Grab pb443) & \multirow{2}{*}{$\begin{array}{c}\text { Zeitscheibe } 2 \\
(3500-3350 \mathrm{BC})\end{array}$} \\
\hline & 3500-3350 (Grab pb385) & \\
\hline & $3370-3090$ (Grab pb388) & Zeitscheiben 2-3 \\
\hline & $3350-3020$ (Grab pb358) & \multirow{3}{*}{$\begin{array}{c}\text { Zeitscheibe } 3 \\
(3350-3100 \mathrm{BC})\end{array}$} \\
\hline & 3320-2920 (Grab pb364) & \\
\hline & 3100-2920 (Grab pb390) & \\
\hline \multirow{5}{*}{ Zeitraum 2} & 3090-2920 (Grab bk383) & \multirow{4}{*}{$\begin{array}{l}\text { Zeitscheibe } 4 \\
(3100-2900)\end{array}$} \\
\hline & 3020-2920 (Grab bk19) & \\
\hline & 3010-2880 (Grab bk75) & \\
\hline & 2900-2700 (Grab bk174) & \\
\hline & $2880-2670(\mathrm{Grab}$ bk158) & \\
\hline
\end{tabular}

\subsection{Relativchronologie der Befunde in den untersuchten Nekropolen}

\subsubsection{Pilismarót-Basaharc}

Hier konnten 42 Bestattungen im Verhältnis zu mGruppen zugeordnet werden (Diagramm 3).

Die Hälfte, 50,0 \% $(n=21)$ der Befunde vertritt die Periode 1 (Grab pb336, pb340, pb343, pb355, pb358, pb359, pb382, pb384, pb386, pb393, pb399, pb406, pb413, pb414, pb419, pb427, pb434, pb436, pb441, pb447, pb448). 45,24 \% ( $\mathrm{n}=19)$ der Bestattungen stammen aus den Perioden 2-6 (Grab pb338, pb356, pb362, pb364, pb400, pb401, pb403, pb439, pb422, pb344, pb429, pb306, pb443, pb352, pb390, pb385, pb351, pb387, pb409). Im Zeitraum der Perioden 1 bis 6 erscheinen Gräber nur von Pilismarót-Basaharc. Nur das Grab pb347 (2,38 \%) kann in die Periode 9 zugestellt werden. Ebenso eine Bestattung datiert in die Periode 11 (Grab pb365).

Das Vorkommen der durch mGruppen seriierten Gräber zeigt Gräberfeldsplan 1.

Die Bestattungen der Periode 1 erscheinen in fast allen Bereichen des Gräberfeldes. Sie bilden eine größere Gruppierung in der südöstlichen Ecke, wo sie dicht beieinander liegen (Grab pb406, pb413, pb414, pb441, pb447, pb448). Zwei andere (Grab pb419, pb 434) befinden sich nördlich von ihnen. Die Befunde der diskutierten Periode treten auch in der westlichen Hälfte der Nekropole auf. Sechs Bestattungen (Grab pb384, pb382, pb358, pb359, pb340, pb343) bilden auch hier eine zusammenhängende Gruppe und zwei Gräber an der westlichen Peripherie (Grab pb427, pb355) liegen ebenso einander nahe. Man kann voraussetzen, dass die Belegung des Gräberfeldes mit diesen Bestattungen angefangen haben dürfte.

Die Gräber der Perioden 2-6 bilden eine größere Gruppe inmitten des Gräberfeldes (Grab pb403, pb409, pbb401, pb422, pb362, pb384, pb387). Drei von ihnen (Grab pb422, pb387, pb384) befinden sich bei den Befunden der Periode 1. Dieses Phänomen kann auch im westlichen Bereich der Nekropole beobachtet werden, wo die Bestattungen dieser Perioden eine größere Gruppe darstellen. 
Tabelle 7. Vorkommen der gemäß Typen seriierten Gräber in den untersuchten Nekropolen

\begin{tabular}{|c|c|c|c|c|c|c|}
\hline $\begin{array}{c}\text { Perioden } \\
\text { (Typen) }\end{array}$ & Pilismarót & Balatonlelle & Budakalász & Fonyód & Mezőcsát & Alsónémedi \\
\hline 1. & $\begin{array}{c}22 \\
(62,86 \%)\end{array}$ & $\begin{array}{c}1 \\
(8,33 \%)\end{array}$ & - & - & - & - \\
\hline 2. & $\begin{array}{c}2 \\
(5,71 \%) \\
\end{array}$ & - & - & - & - & - \\
\hline 3. & \multirow{8}{*}{$\begin{array}{c}8 \\
(22,86 \%)\end{array}$} & - & - & - & - & - \\
\hline 4. & & - & - & - & - & - \\
\hline 5. & & - & - & - & - & - \\
\hline 6. & & - & - & - & - & - \\
\hline 7. & & - & - & - & - & - \\
\hline 8. & & - & - & - & - & - \\
\hline 9. & & - & - & - & - & - \\
\hline 10. & & - & - & - & - & - \\
\hline 11. & $\begin{array}{c}2 \\
(5,71 \%) \\
\end{array}$ & - & $\begin{array}{c}1 \\
(5,0 \%) \\
\end{array}$ & - & - & - \\
\hline 15. & $\begin{array}{c}1 \\
(2,86 \%)\end{array}$ & - & $\begin{array}{c}8 \\
(40,0 \%) \\
\end{array}$ & - & - & - \\
\hline 14. & - & $\begin{array}{c}8 \\
(66,67 \%) \\
\end{array}$ & $\begin{array}{c}7 \\
(35,0 \%) \\
\end{array}$ & - & $\begin{array}{c}1 \\
(16,67 \%)\end{array}$ & - \\
\hline 13. & - & $\begin{array}{c}3 \\
(25,0 \%)\end{array}$ & $\begin{array}{c}3 \\
(15,01 \%)\end{array}$ & - & $\begin{array}{c}5 \\
(83,33 \%)\end{array}$ & 1 \\
\hline 12. & - & - & $\begin{array}{c}1 \\
(5,0 \%)\end{array}$ & 1 & - & - \\
\hline Insgesamt & 35 & 12 & 20 & 1 & 6 & 1 \\
\hline
\end{tabular}

Tabelle 8. Zeiträume gemäß Typen im Verhältnis zu den Radiokarbondaten (Pilismarót-Basaharc und Budakalász)

\begin{tabular}{|c|c|c|}
\hline Belegungsphasen gemäß Typen & $\begin{array}{c}\text { Radiokarbondaten } \\
\text { (cal BC) }\end{array}$ & $\begin{array}{c}\text { Zeitscheiben } \\
\text { nach FuRHOLT }(2009,2011)\end{array}$ \\
\hline \multirow{6}{*}{ Zeitraum 1} & $3500-3350(\mathrm{pb} 443)$ & \multirow{2}{*}{$\begin{array}{l}\text { Zeitscheibe } 2 \\
(3500-3350 \mathrm{BC}) \\
\end{array}$} \\
\hline & $3500-3350(\mathrm{pb} 385)$ & \\
\hline & $3370-3090(\mathrm{pb} 388)$ & \multirow{5}{*}{$\begin{array}{l}\text { Zeitscheiben 2-3 } \\
(3350-3100 \mathrm{BC})\end{array}$} \\
\hline & 3360-3090 (pb399) & \\
\hline & $3350-3020(\mathrm{pb} 358)$ & \\
\hline & $3330-2920(\mathrm{pb} 364)$ & \\
\hline \multirow{4}{*}{ Zeitraum 2} & $3100-2920(\mathrm{pb} 390)$ & \\
\hline & 3090-2920 (bk383) & \multirow{3}{*}{$\begin{array}{l}\text { Zeitscheibe } 4 \\
(3100-2900 \mathrm{BC})\end{array}$} \\
\hline & 3020-2900 (bk378) & \\
\hline & $3010-2880$ (bk75) & \\
\hline
\end{tabular}

Das Grab pb347 datiert schon in die Periode 9 an der südlichen Peripherie des westlichen Gräberfeldteils. Das Grab pb365 nicht weit von dem Vorigen dürfte als der jüngste Befund gemäß mGruppen behandelt werden.

Die Seriation gemäß Typen ergab eine viel engere chronologische Gruppierung der Befunde. Es handelt sich dabei um 35 Gräber, die nur in 5 Perioden datieren (Diagramm 4).

Mehr als die Hälfte, 62,86 \% $(\mathrm{n}=22)$ der Befunde kann in die Periode 1 zugestellt werden $(\mathrm{Grab}$ pb336, pb343, pb351, pb352, pb355, pb358, pb359, pb382, pb384, pb386, pb413, pb414, pb419, pb426, pb427, pb430, pb434, pb436, pb441, pb443, pb444, pb449).

Nur 5,71 \% ( $\mathrm{n}=2)$ der Gräber stammt aus der Periode 2 (Grab pb400, pb364). Die Perioden 3-10 (22,86\%, $\mathrm{n}=8$ ) sind durch je eine Bestattung repräsentiert (Grab pb422, pb439, pb388, pb403, pb339, pb385, pb344, pb338). 
$5,71 \%(\mathrm{n}=2)$ der Befunde kann in die Zeit der Periode 11 datiert werden (Grab pb401, pb390). Ein beachtenswertes Phänomen ist, dass eine Bestattung (Grab pb347) (2,86 \%) in die jüngste Periode (Periode 15) datiert.

Das Vorkommen der durch die Typen seriierten Gräber in der Nekropole von Pilismarót-Basaharc zeigt Gräberfeldsplan 2.

Die Gruppenbildung der durch Typen seriierten Gräber der Periode 1 zeigt ein ähnliches Bild wie im Fall der Gruppenbilung durch mGruppen. Die Befunde pb443 (Typen 1b2c2, 2d1a1, 2d2a5), pb444 (Typen 1b1a1,

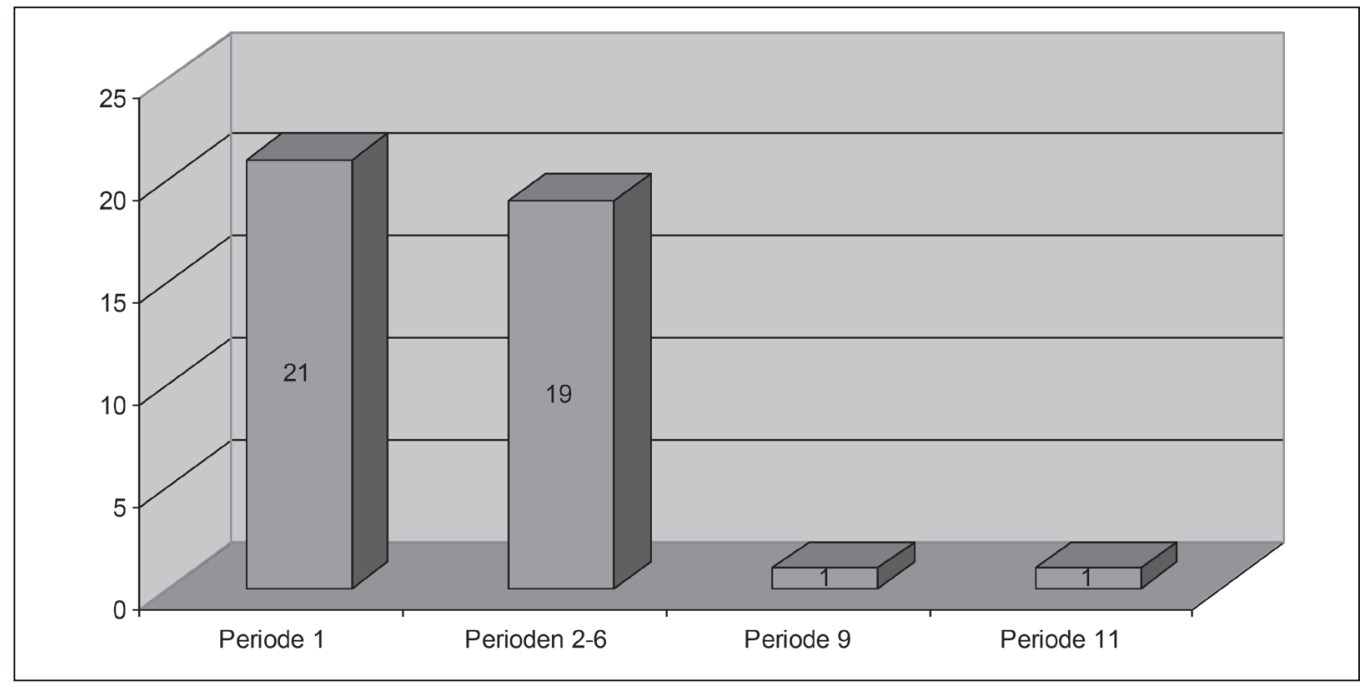

Diagramm 3. Verteilung der Gräber in Pilismarót-Basaharc auf Grund der durch mGruppen definierten Perioden

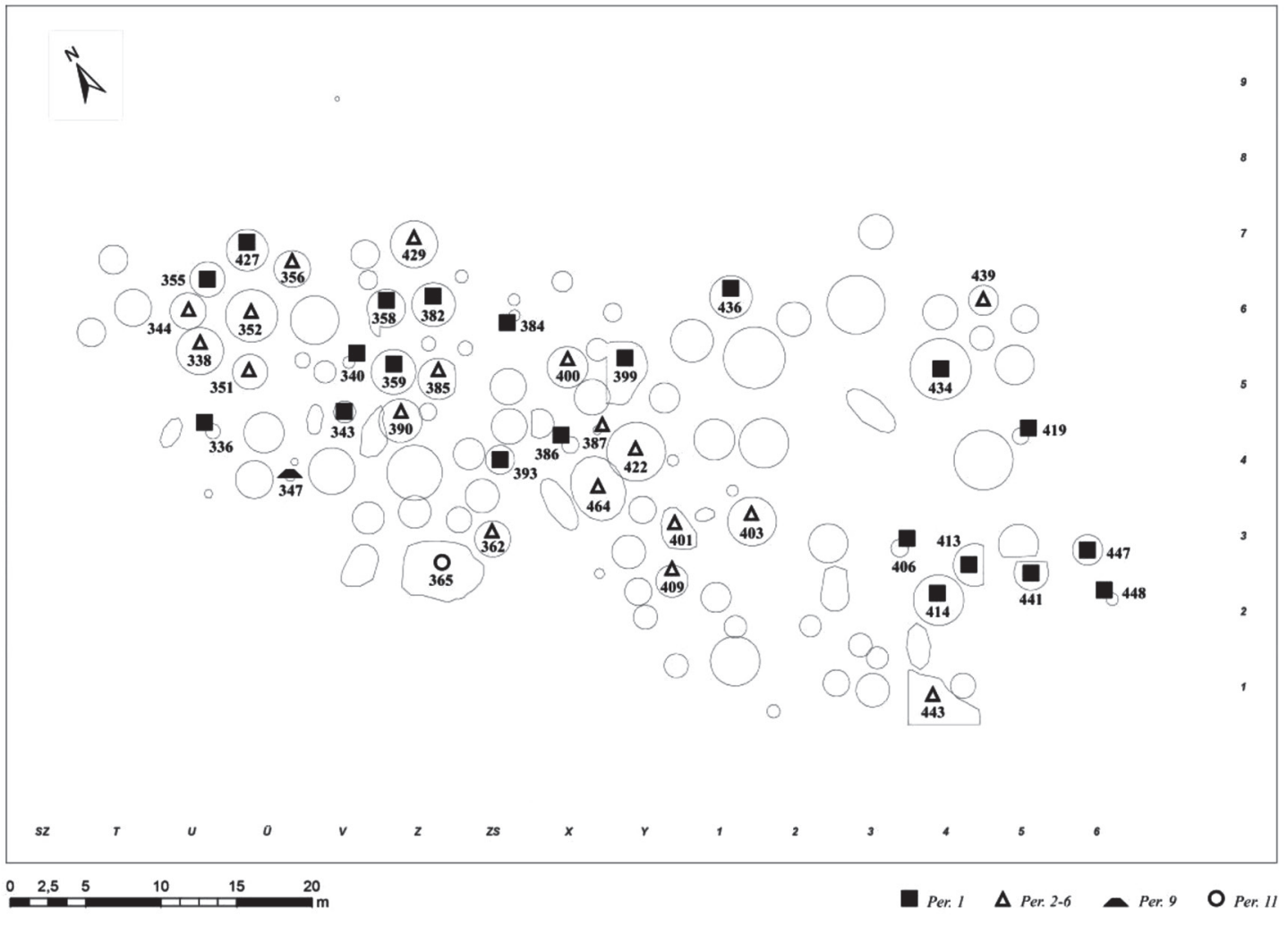

Gräberfeldsplan 1. Verbreitung der durch mGruppen seriierten Gräber in Pilismarót-Basaharc 
1b2b4, 1e3a4, 2c1d4, 2f1c1) und pb449 (Typen 2d2a5, 2d2a7) bzw. pb414 (Typen 1d1b4, 3b1a1), pb413 (Typen 1d1b2, 3b1a1) und pb441 (Typen 1b2b4, 2a4b2, 2e1a1) bilden eine getrennte Gräbergruppe mit zwei kleineren Gruppierungen im südöstlichen Teil der Nekropole.

Die Gräber pb419 (Typen 2a4b1, 2c1d8, 2f1c3) und pb434 (Typen 1b2b1, 1d1b4, 1d2a1, 2a4b2) erscheinen nördlich von den Vorigen einander nahe, wie pb436 (Typen 1c1a1, 2c1d1, 2d2a5) und pb426 (Typen 1b2b1, 1c1a1) an der nördlichen Peripherie.

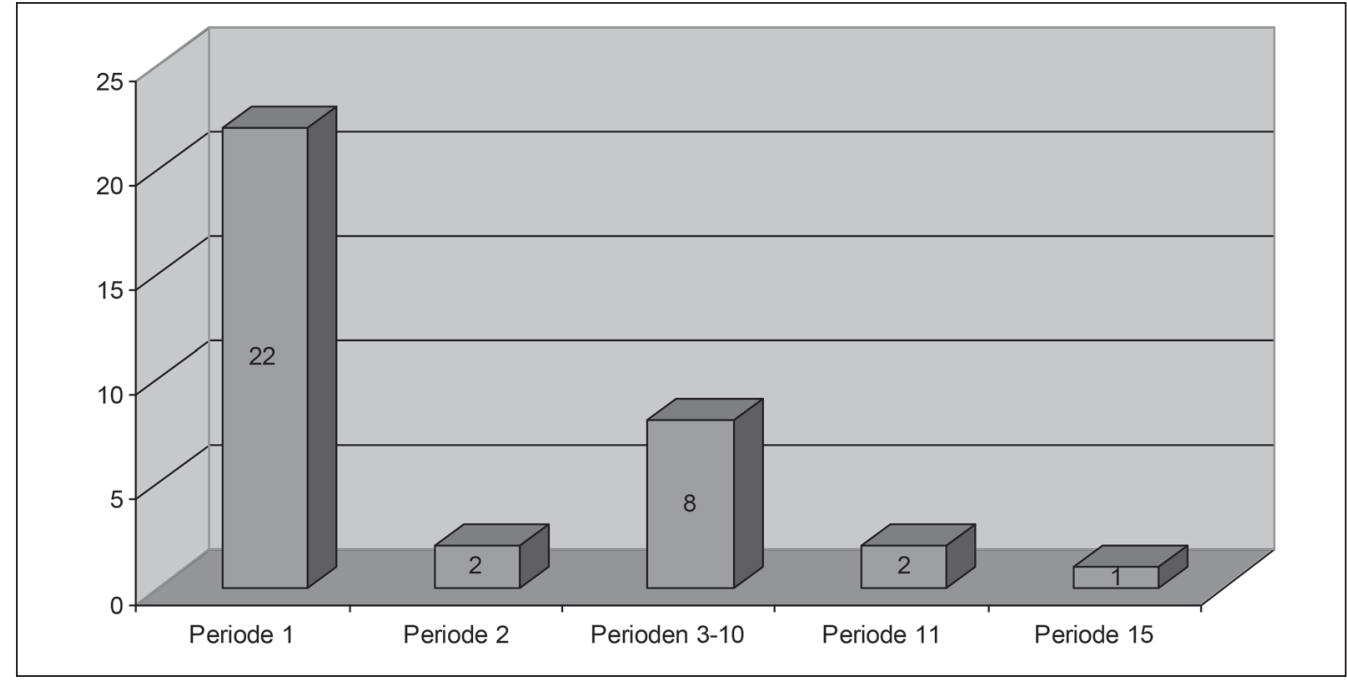

Diagramm 4. Verteilung der Gräber in Pilismarót-Basaharc auf Grund der durch Typen bestimmten Perioden

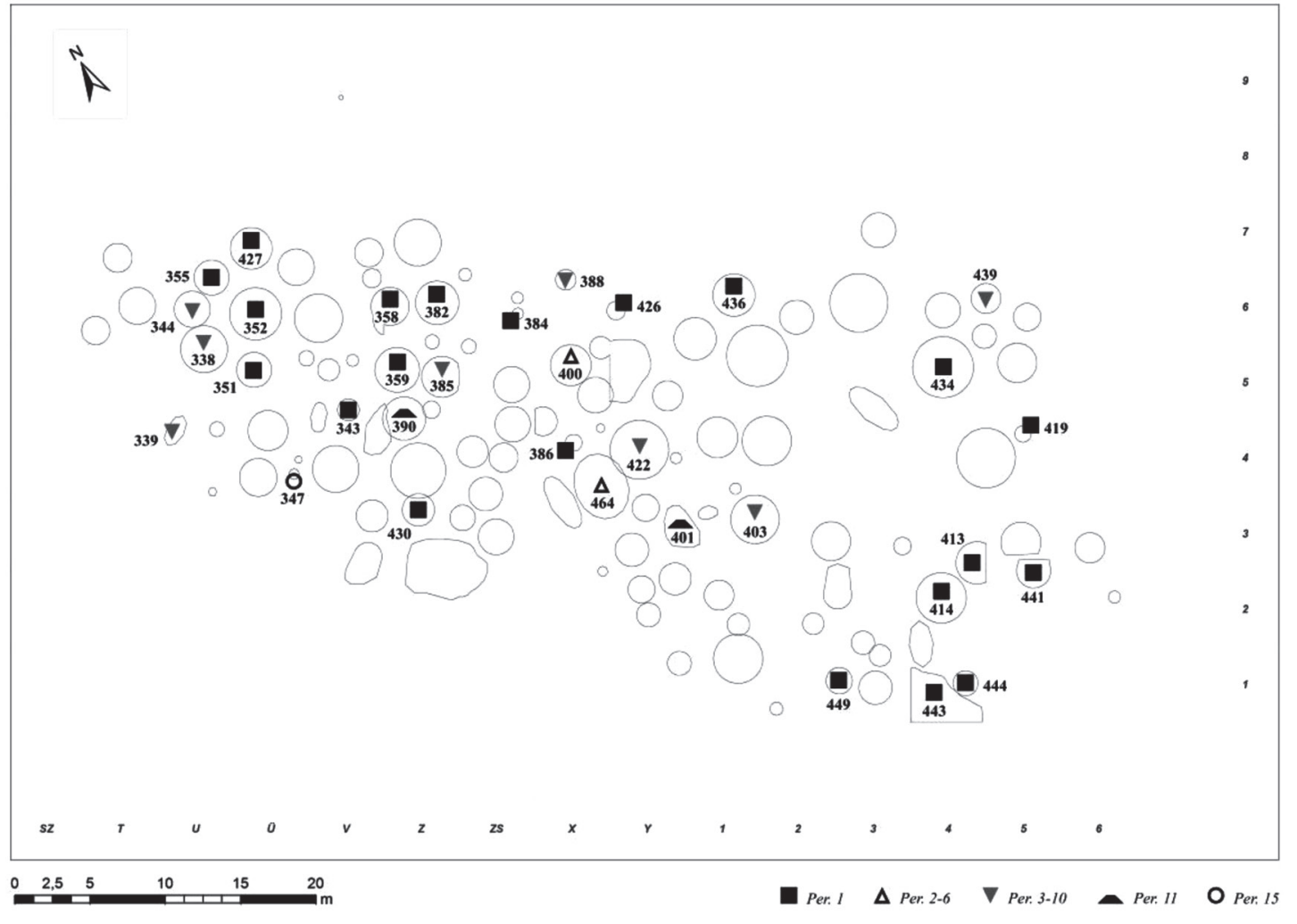

Gräberfeldsplan 2. Verteilung der durch Typen seriierten Bestattungen in Pilismarót-Basaharc 
Auch in der großen westlichen Gräbergruppe liegen die Bestattungen der erörterten Periode unmittelbar beieinander oder einander nahe. Es handelt sich dabei um die Gräber pb384 (Typen 1d1b4, 2a4b2, 2c1d7), pb382 (Typen 1b1b2, 2c1c1), pb358 (Typen 1b2b1, 1b2b4, 1d1b2, 1e3a3, 2c1d4, 2d1a2, 2d2a5), pb359 (Typen 2d2a5, 2e1b2, 3b1a1, 3f1a1), pb343 (Typen 1b1b2, 2c1c1), pb336 (Typen 1b1b2, 1b2b3, 1b2b4, 2c1d8, 2c2a2, 2d1a1, 2f1c1, 2f1c3), pb351 (Typen 1d1a2, 2d1a2, 2d2a10), pb352 (Typen 1b3b1, 2c1c1), pb355 (Typen 2c1c1, 2d2a5, 2e1b2) und pb427 (Typen 1b1b1, 2f1c3, 3e1a1).

Der Befund pb388 (Typen 1b1a3, 1c1a1, 1c4a1, 2d2a3, 2f1a1, 2f1c3, 3d1a1) kam etwa im mittleren Teil des Gräberfeldes zum Vorschein.

Beachtenswert ist auch diesmal, dass die Bestattungen pb400 (Typen 1b3b1, 1e3a2, 2c1c1, 2d2a10) und pb364 (Typen 2c2a1, 2d2a7) der Periode 2 in der Mitte der Nekropole zwischen oder bei den Gräbern der Periode 1 und der Perioden 3-10 liegen.

Die Befunde der Perioden 3-10 verteilen sich zudem auf einer größeren Fläche im Gräberfeld, weit voneinander. Nur die Gräber pb344 (Typen 1b2b2, 1d1c1, 2d2a10) und pb339 platzieren am Westrand der Nekropole beieinander bzw. einander nahe. Die Bestattungen pb388 (Typen 1b1a3, 1c1a1, 1c4a1, 2d2a3, 2f1a1, 2f1c3, 3d1a1), pb385 (Typen 1b3b1, 2c1b2, 2d2a3), pb422 (Typen 1b3b1, 2c1d7, 2d1a1, 2d2a9) und pb403 (Typen 1b2b3, 1d2a3, 2d2a3, 2d2a7, 2e1b1) sind etwa im mittleren Bereich des Gräberfeldes zu sehen, sie bilden dagegen keine zusammenhängende Gruppe untereinander. Das Grab pb439 (Typen 1b1a3, 2c1d4, 2c2a1, 2d2a5, 3a1a1) dieser Periode befindet sich an der nordöstlichen Peripherie des Gräberfeldes.

Die jüngsten Befunde der Periode 11 und 15 sind im mittleren Bereich der Nekropole weit voneinander, bei denen der vorigen Periode zu finden. Das Grab pb401 der Periode 11 (Typen 2c1d5, 2d2a3, 3a1a1) liegt etwa in der Mitte des Gräberfeldes, bei den Befunden der vorigen Periode bzw. ihnen nahe. Auch die Bestattung pb390 (Typen 1c2c1, 1e3b1, 2c1d5) liegt in der großen Westgruppe unmittelbar neben einem Grab der Perioden 3-10.

Das Grab pb347 (Typen 1d1a3, 2f1a3) dürfte eventuell das jüngste gewesen sein. Es befindet sich an der südlichen Peripherie der Westgruppe.

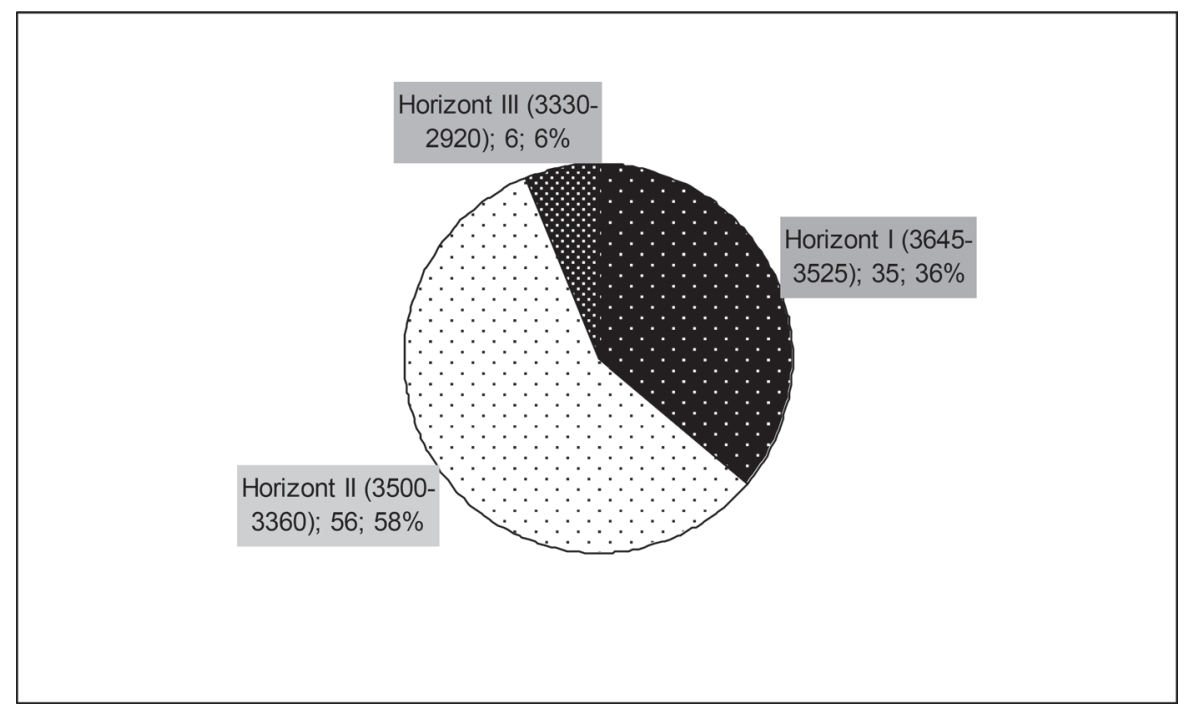

Diagramm 5. Verteilung der Gräber von Pilismarót-Basaharc gemäß Horizonten nach M. Bondár (2015)

M. Bondár bestimmte drei Belegungshorizonte in der Nekropole von Pilismarót-Basaharc. 36,08\% $(\mathrm{n}=35)$ der Gräber wurde zeitlich in den Horizont I (3645-3525), 57,73 \% $(\mathrm{n}=56)$ in den Horizont II (3500-3360 BC) und 6,19\% $(\mathrm{n}=6)$ in den Horizont III gestellt (Diagramm 5). ${ }^{86}$

Die Übereinstimmungen oder Abweichungen zwischen den Bestimmungen durch Horizonte und Seriation gemäß Typen sind im Fall von 34 Gräbern bekannt (Diagramm 6). 21 Gräber aus dem Horizont I (Grab pb343,

${ }^{86}$ BONDÁR 2015, 245-251, Tab. 10. 


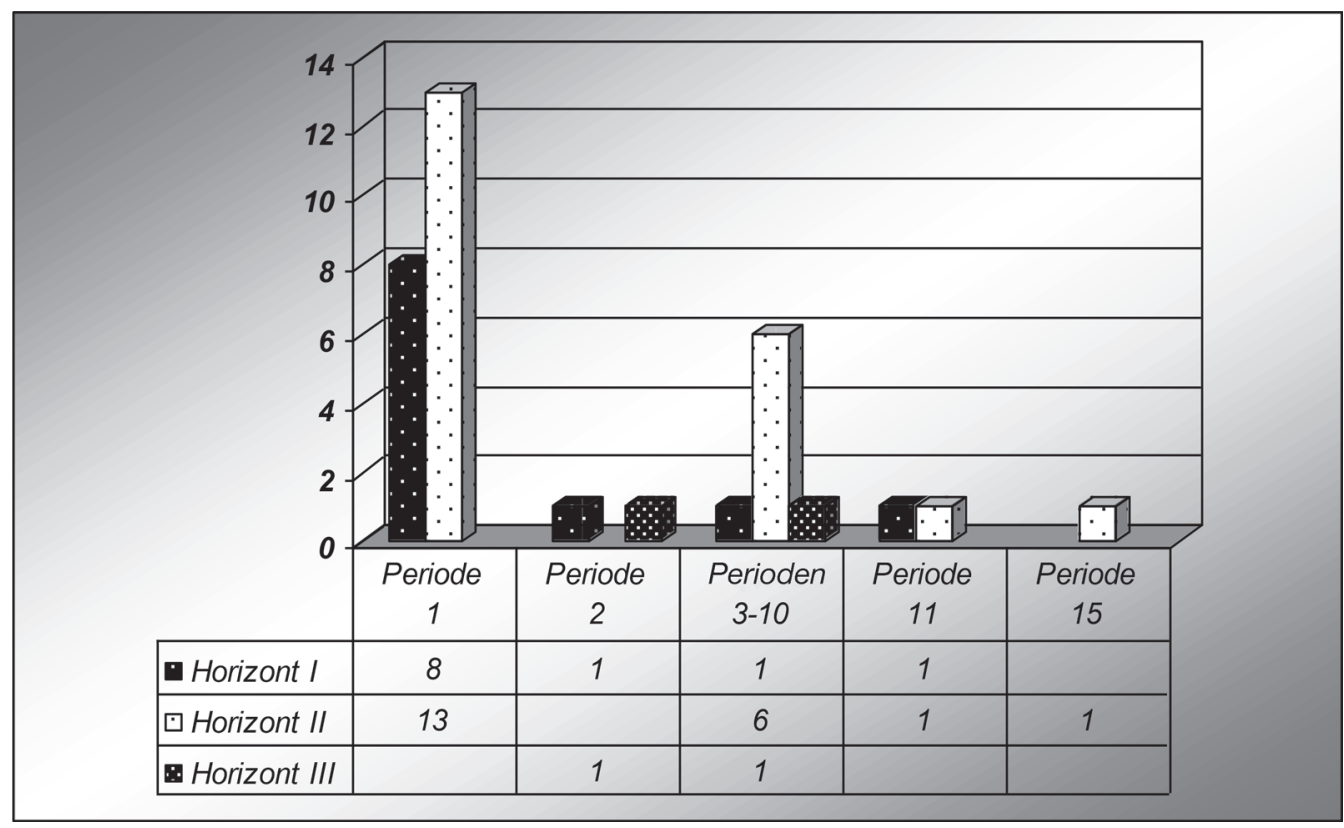

Diagramm 6. Zusammenhänge zwischen den Horizonten nach M. Bondár (2015) und den Perioden durch Seriation gemäß Typen

pb351, pb441, pb352, pb355, pb384, pb419, pb434) und dem Horizont II (Grab pb336, pb358, pb359, pb382, pb386, pb413, pb414, pb426, pb427, pb436, pb443, pb444, pb449) wurden der Periode 1 zugeteilt. Je eine Bestattung des Horizontes I (Grab pb409) und III (Grab pb364) wurden als Befunde der Periode 2 bestimmt. Ebenso je eine Bestattung des Horizontes I (Grab pb344) und III (Grab pb388) vertritt die Perioden 3-10, während sechs andere derselben Periode als Befunde des Horizontes II definiert wurden (Grab pb338, pb339, pb385, pb403, pb422, pb439). Das Grab pb401 des Horizontes I und das Grab pb390 des Horizontes II sollen aus der Periode 11 gestammt haben. Das Grab pb347 kennzeichnet den Horizont II, durch die Seriation wurde es dagegen in die Periode 15 zugeordnet.

Resümee zu Pilismarót-Basaharc. Die Belegung/Benutzung des Gräberfeldes von Pilismarót-Basaharc dürfte mit den Bestattungen der Periode 1 der südöstlichen Grabgruppierung und der größeren westlichen Gräbereinheit angefangen haben. Die Keramik weist ähnliche Merkmale in diesen Gräbern auf, jene der südöstlichen Gruppierung scheinen dagegen etwas älter zu sein. Auf Grund der Charakteristik der Keramikgefäße dürfte ein zeitlicher Bruch zwischen den Perioden 1-2 und 3-10 vorausgesetzt werden. Zur Zeit der Perioden 3-10 sind bedeutsame Änderungen der Charakteristik der Grabkeramik nachweisbar, es treten sogar neue Gefäßformen auf. Bemerkt werden muss zugleich, dass die Bestattungen dieser Periode auf den verschiedenen Teilen der Nekropole liegen. Sie bilden (mit einer Ausnahme) keine zusammenhängenden Gruppierungen untereinander und befinden sich immer bei den Bestattungen der vorangehenden Periode oder nahe ihnen.

In der Periode 11 treten wieder neue Gefäßtypen auf. Aber auch in diesem Fall ist es zu bemerken, dass auch diese jüngsten Bestattungen bei denen der vorigen Periode oder nahe ihnen liegen. Ein zeitlicher Bruch dürfte eventuell zwischen der Periode 11 und der Periode 15 vorausgesetzt werden.

\subsection{Budakalász}

Gemäß den mGruppen konnten 39 Bestattungen im Gräberfeld von Budakalász zeitlich eingeordnet werden. Sie vertreten sieben Perioden (Diagramm 7).

Je 5,13\% $(\mathrm{n}=2-2)$ der Bestattungen von Budakalász dürfte aus der Periode 7 (Grab bk13, bk378) und der Periode 9 (Grab bk23, bk367) gestammt haben. Je 10,26 \% ( $\mathrm{n}=4-4)$ der Befunde datiert in die Periode 8 (Grab 
bk210, bk248, bk345, bk350) und 10 (Grab bk273, bk340, bk344, bk383), während 20,51\% (n=8) von ihnen der Periode 11 zugeordnet werden kann (Grab bk59, bk70, bk158, bk286, bk335, bk339, bk347, bk368). Die Mehrheit, 33,33\% ( $\mathrm{n}=13)$ der Gräber von Budakalász vertritt die Periode 12 (Grab bk18, bk36, bk80, bk112, bk118, bk132, bk142, bk164, bk189, bk197, bk202, bk227, bk360). 15,38 \% (n=6) wurde zur Zeit der jüngsten Perioden 13-15 angelegt (Grab bk3, bk75, bk33, bk174, bk19, bk203).

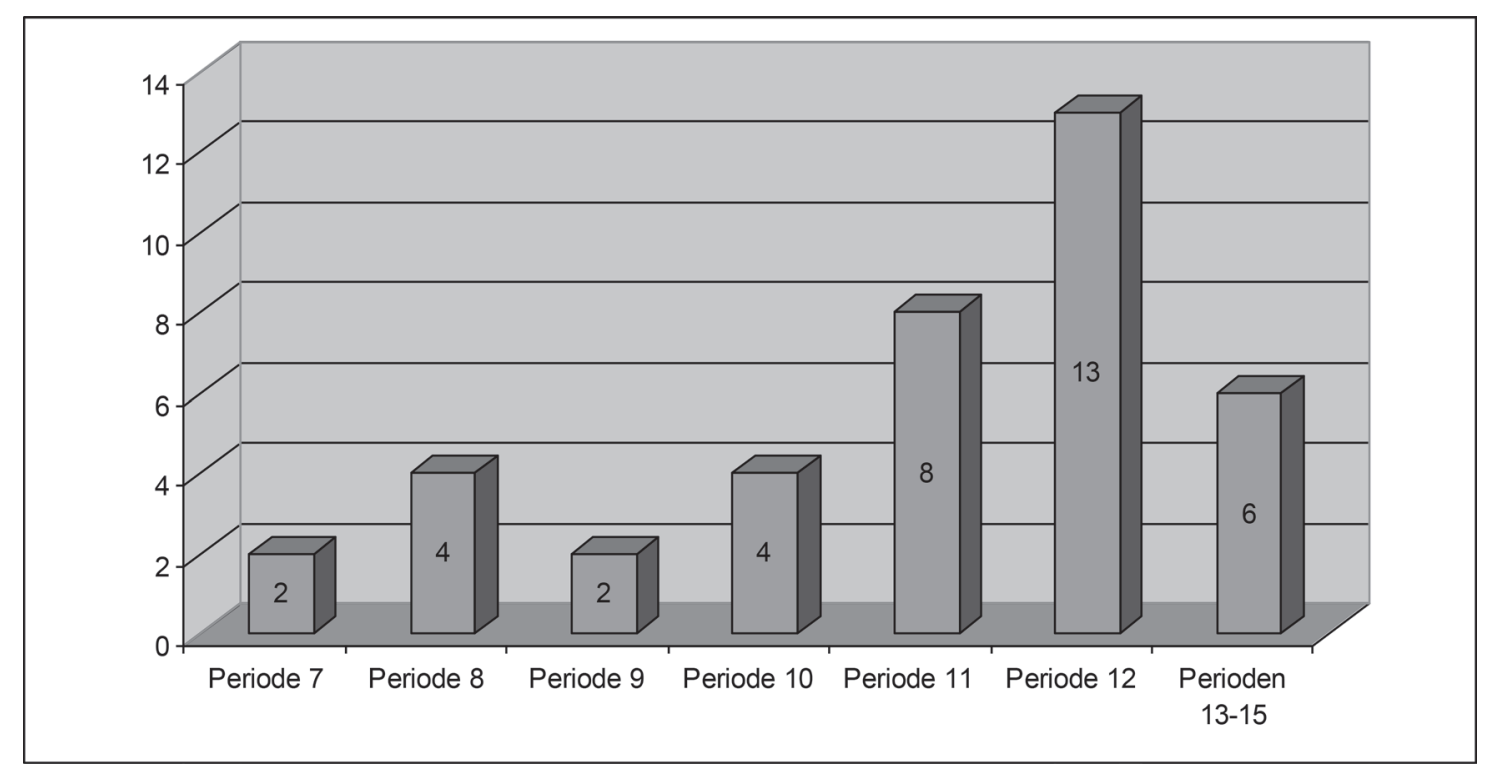

Diagramm 7. Verteilung der Gräber in Budakalász auf Grund der durch mGruppen definierten Belegungsphasen

Das Vorkommen der durch mGruppen seriierten Bestattungen gemäß Perioden zeigt Gräberfeldsplan 3. Es gibt 20 Gräber, die gemäß Typen gruppiert werden konnten. In diesem Gräberfeld sind nur die jüngsten fünf Perioden vertreten (Diagramm 8).

Je 5,0 \% ( $\mathrm{n}=1)$ der Gräber stammt aus der Periode 11 (Grab bk210) und aus der Periode 12 (Grab bk367). $15,0 \%(n=3)$ der Befunde gehört der Periode $13 \mathrm{zu}$ (Grab bk142, bk189, bk222). Bestattungen aus der Zeit der Periode 14 sind durch 35,0 \% ( $\mathrm{n}=7)$ vertreten (Grab bk197, bk345, bk330, bk350, bk312, bk248, bk273). Im größten Anteil, in 40,0 \% ( $\mathrm{n}=8)$, wurden hier Gräber der finalen Zeit, der Periode 15, festgestellt (Grab bk378, bk80, bk75, bk339, bk68, bk59, bk383, bk347). plan 4 dar.

Die räumliche Verteilung der durch Typen seriierten Bestattungen in der Nekropole stellt Gräberfelds-

Als der älteste Befund dürfte das Grab bk210 gemäß Seriation durch Typen auf der südöstlichen Peripherie der Nekropole behandelt werden. Dieses Grab vertritt die Periode 11 und besitzt einen dreigliedrigen Becher vom Typ 1c3a2 sowie eine profilierte Schüssel vom Typ 2d2a1.

Das Grab bk367 aus der Periode 12 liegt auf dem östlichen Rand des Gräberfeldes und weist die Typen 1b5b6 der Henkelbecher, 2a2a1 und 2a2a6 der Henkeltassen bzw. 2d2a1 der profilierten Schüsseln auf.

Ein auffallendes Phänomen ist, dass sich die drei Gräber der Periode 13 im zentralen Bereich, sogar einander nahe befinden. Es handelt sich dabei um das Grab bk142 mit den Typen 1a3a2 der Fußgefäße, 1b3a3 der Henkelbecher und 2a4d1 der Henkeltassen, das Grab bk189 mit den Typen 1c3a4 der dreigliedrigen Becher und 2a2a1 bzw. 2a4d1 der Henkeltassen und das Grab bk222 mit dem Fußgefäßtyp 1a2a1, den Henkelbechertypen $1 \mathrm{~b} 3 \mathrm{a} 3$ und 1b3b4 sowie dem Henkeltassentyp 2a2a5.

Auch die Raumposition der Befunde der Periode 14 gemäß Typen ist beachtenswert. Die Gräber bk197 (Typen 1a3a1, 1b2a3, 2a3b3, 2a4d1), bk248 (Typen 1b6b2, 2e1a3), bk273 (Typen 1b3b2, 1b4b4, 1e2a1), bk312 (Typen 1b2a2, 1b3b6, 1b6b1, 2a4d1, 2f1b1) und bk345 (Typen 1b3b4, 2f1a2) liegen entlang einer leicht geschweiften Linie in der nördlichen Hälfte des Gräberfeldes, nicht weit voneinander. Die Bestattungen bk330 (Typen 1a4a1, 


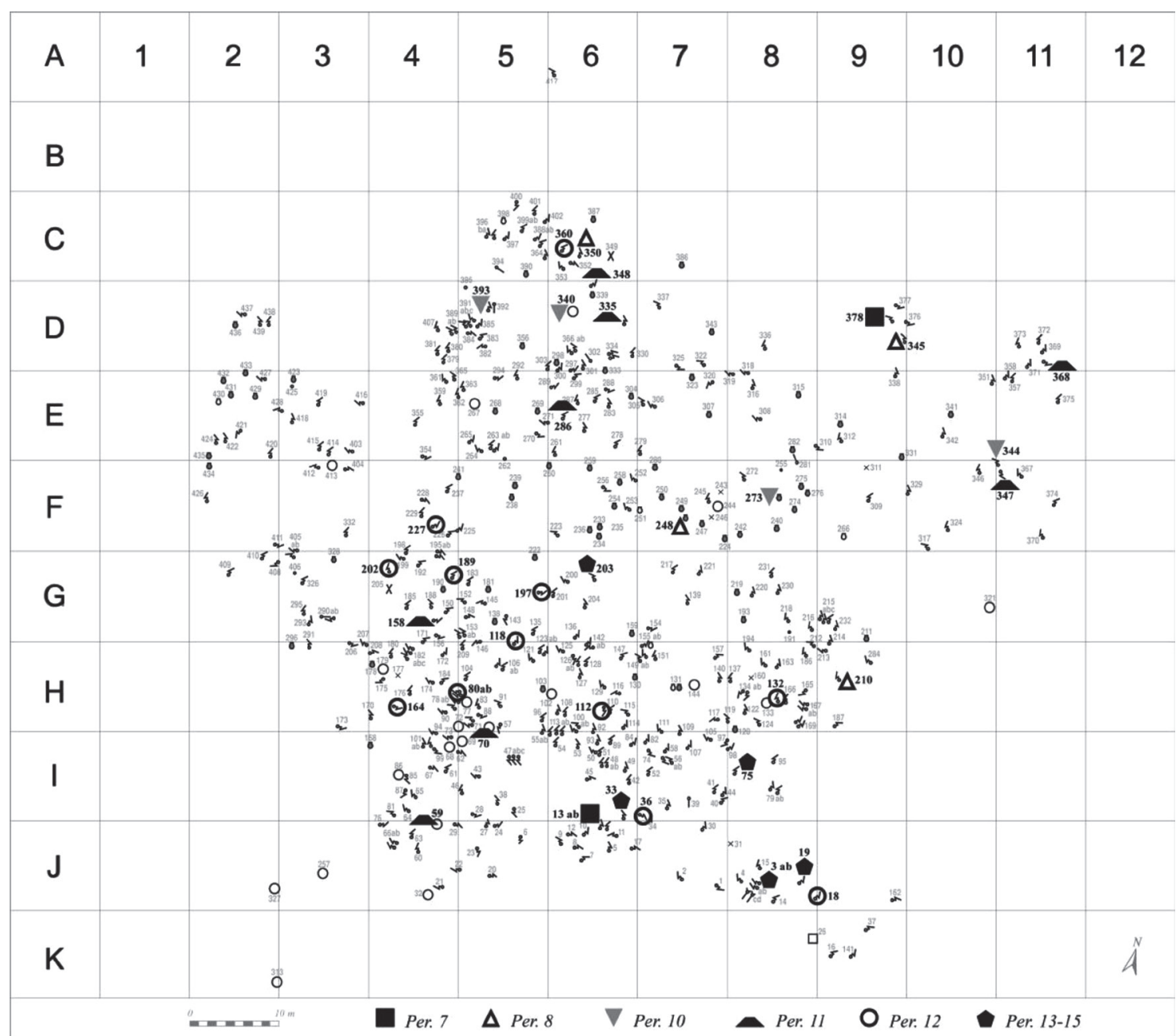

Gräberfeldsplan 3. Verteilung der durch mGruppen seriierten Bestattungen in Budakalász

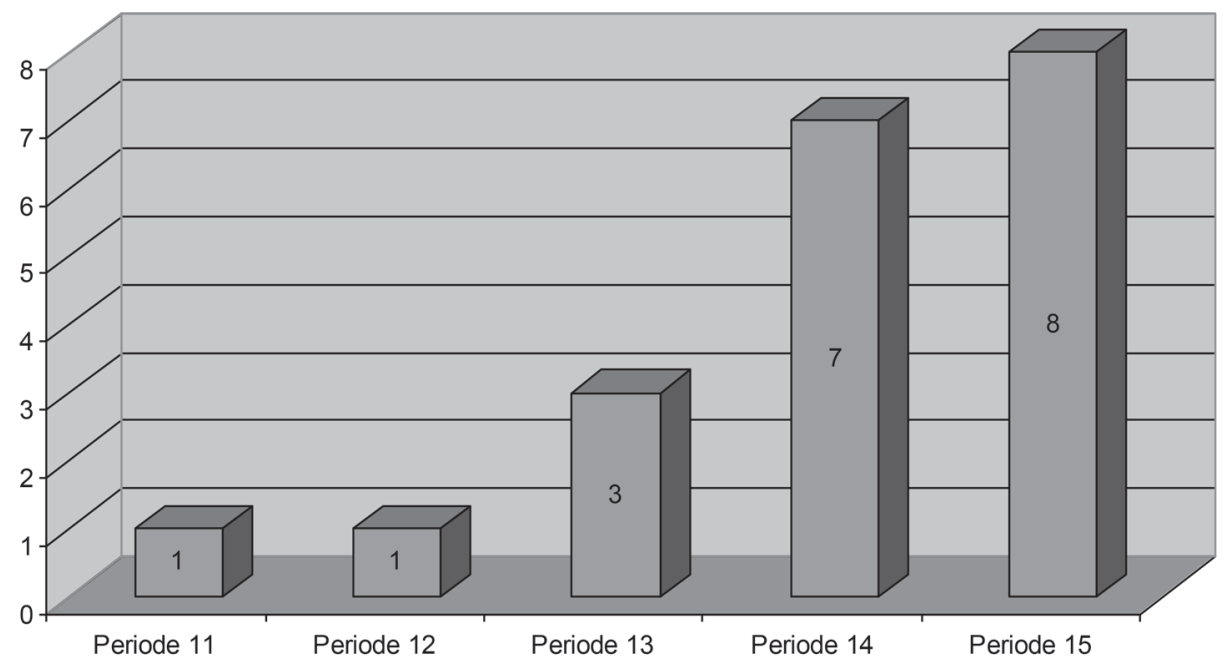

Diagramm 8. Verteilung der Gräber in Budakalász auf Grund der durch Typen definierten Perioden 


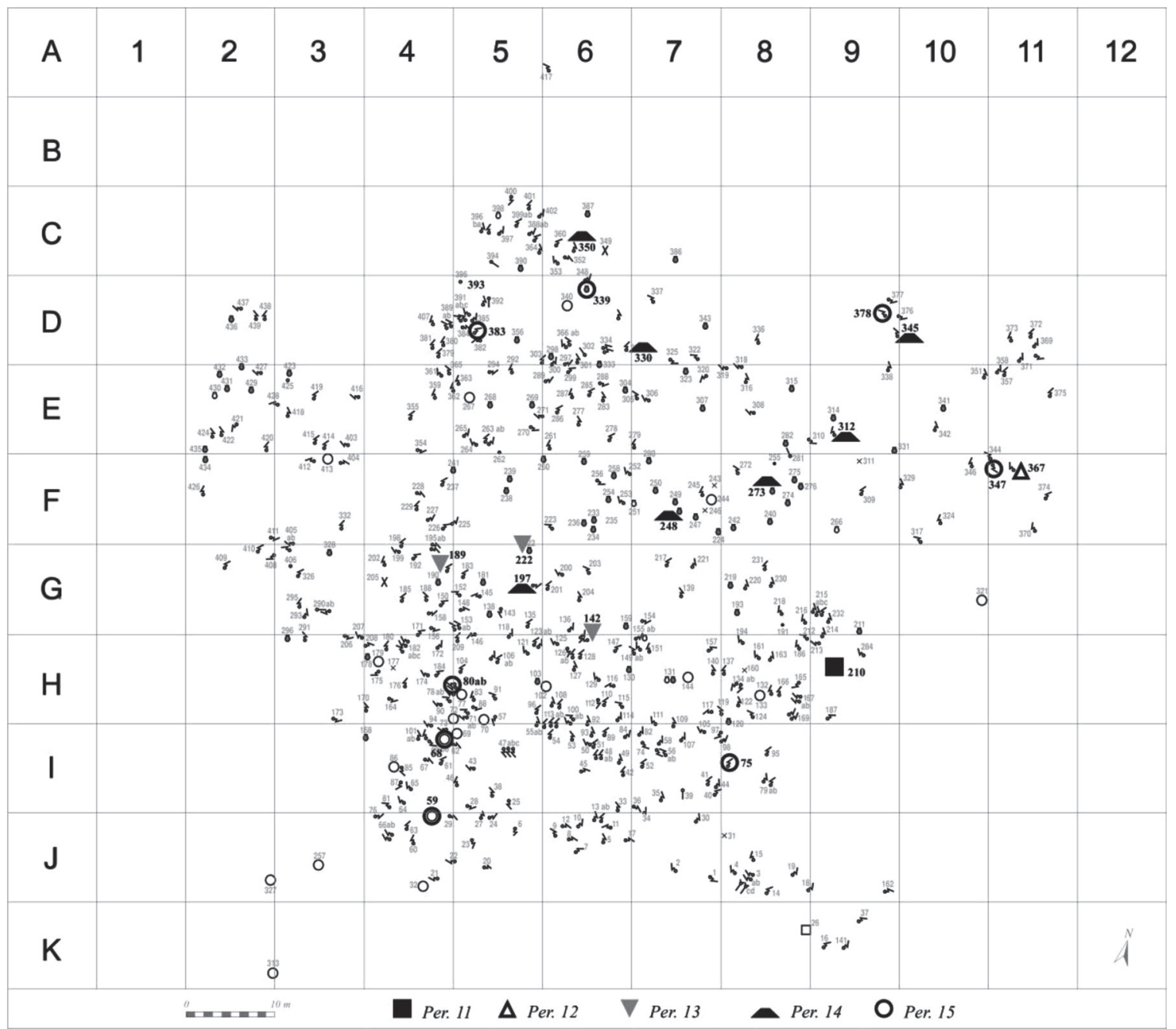

Gräberfeldsplan 4. Verteilung der durch Typen seriierten Bestattungen in Budakalász

1b1a4, 2f1a2) und bk350 (Typen 1b3b2, 2f1a2) befinden sich im nördlichen Bereich der Nekropole nicht weit voneinander.

Die Mehrheit der Gräber der Periode 15 befindet sich an der Peripherie des Gräberfeldes. So liegt das Grab bk378 (Typen 1a1b1, 1b4c3, 1d1b1, 2a4a2) auf dem nordöstlichen Teil bei dem Grab bk345 der vorigen Periode. Die Bestattung bk347 (Typen 1b4a3, 2b1a1, 2f1a2) befindet sich südlich davon, unmittelbar neben dem Grab bk367 der Periode 12. Das Grab bk75 (Typen 1a1a2, 1a1a4, 1a1b1, 1a3b1, 1b4a1, 1b4a2, 2a3d1, 2a4c2) liegt am südöstlichen Rand. Das Grab bk383 (Typen 1a1b3, 2a3b3, 2f1a2) und Grab bk339 (Typen 1b4c3, 2a3b3, 2f1b2) erscheinen in der nördlichen Gräbergruppe, nicht weit voneinander, im Kreis der Bestattungen der vorigen Periode. Die Gräber bk59 (Typen 1b3a8, 1b5c5, 2a4c2, 2f1a3), bk68 (Typen 1b4a3, 2a4c2) und bk80 (Typen 1b5b3, 2a3d1, 2f1b2) liegen in der südwestlichen Gräbergruppe, nicht weit voneinander.

Als Ergebnis der 14C-Untersuchungen der Gräber von Budakalász stellt Zs. Siklósi Folgendes fest: „It is therefore impossible or hardly possible to determine chronological differences between Graves 403, 230, 142/B, 182/A, 182/C and 252, and the same holds true for Graves 13/A, 383, 182/B, 19 and 378. Otherwise, the very similar uncalibrated dates suggest that there might only be slight chronological differences between them. Grave 182 , a triple burial, confirms this possibility because the calibrated radiocarbon dates for Graves 182/A and 182/C are identical (3330 (68.2\%) 3020 cal BC), while Grave 182/B seems slightly younger (3030 (68.2\%) 2920 cal BC), even though Grave 182/B lay partially under Graves 182/Aand 182/C. [...] Only Grave 75 falls within a longer, 
steep phase of the calibration curve, enabling a more precise dating. This date (3010 (68.2 \%) $2880 \mathrm{cal}$ BC), however, is the last in the compact radiocarbon sequence. Two burials (Graves 174 and 158) seem much younger than the others. These younger dates also lie on a plateau of the calibration curve. The date for Grave 75 might be seen as being intermediate between the younger and the older graves, suggesting that there is no reason for assuming that the young date of these samples can be attributed to contamination or that there was a hiatus between the older and younger graves in the cemetery's use. “87

Resümee zu Budakalász. Als das älteste, durch Typen seriierte Grab von Budakalász können wir die Bestattung bk210 am südöstlichen Rand der Nekrople behandeln. Sie vertritt die Periode 11, d. h. schon die klassische Badener Kultur. Die Gefäßtypen dieses Grabs erscheinen im keramischen Bestand der Periode 1 von PilismarótBasaharc nicht. Eine Ausnahme ist der Typ 2d2a1 der profilierten Schüsseln, aber nur auf dem Niveau der mGruppen (2d2a), und zwar nur auf die Perioden 1 und 2 von Pilismarót-Basaharc folgend.

Der typologische Charakter der Keramikgefäße der Periode 12 bis in die Periode 15 von Budakalász ändert sich nur wenig. Besonders die Keramiken der Perioden 12 und 13 bzw. die der Perioden 14 und 15 weisen ähnliche, sogar übereinstimmende Merkmale auf.

\subsection{Alsónémedi}

Es sind hier nicht mehr als vier Bestattungen, die gemäß mGruppen in vier Perioden (je 25,0 \%) eingeordnet werden konnten. Aus der Periode 8 (Doppelgrab an3 und an5), der Periode 11 (Grab an22), der Periode 12 (Grab an28) und der Periode 14 (Grab an62) stammt je ein Befund (Diagramm 9).

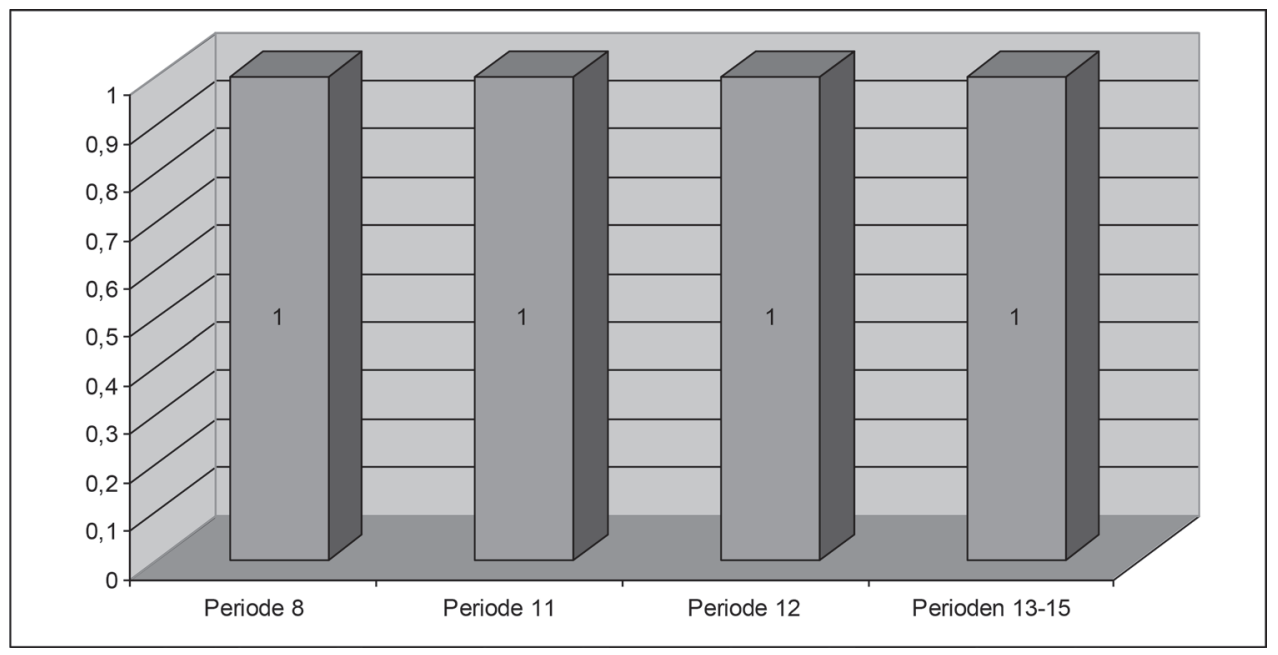

Diagramm 9. Verteilung der Gräber in Alsónémedi auf Grund der durch mGruppen definierten Perioden

Das Vorkommen der durch mGruppen seriierten Gräber in der Nekropole von Alsónémedi illustriert Gräberfeldsplan 5.

In diesem Fall konnte die relativchronologische Lage nur bei vier Gräbern bestimmt werden. Der älteste Befund (Doppelgrab an3 und an5) aus der Periode 8 liegt im Zentrum des Gräberfeldes. Die Bestattung an28 der Periode 11 kommt im nordöstlichen Teil vor und besitzt die Henkelbechertypen 1b3a7 und 1b7a1, 2b1a3 von zweigeteilten Schüsseln sowie den Löffeltyp 2g2a3. Im Grab an22 der Periode 12 befanden sich eine Henkeltasse (Typ 2a3b2) und eine profilierte Schale (Typ 2f2c2). Südlich von den anderen wurde die im Grab an62 der Periode 14 bestattete Person mit zwei Henkelbechern (Typen 1b4a4 und 1b6a2) versehen.

${ }^{87}$ SiKLósi 2009, 458. 


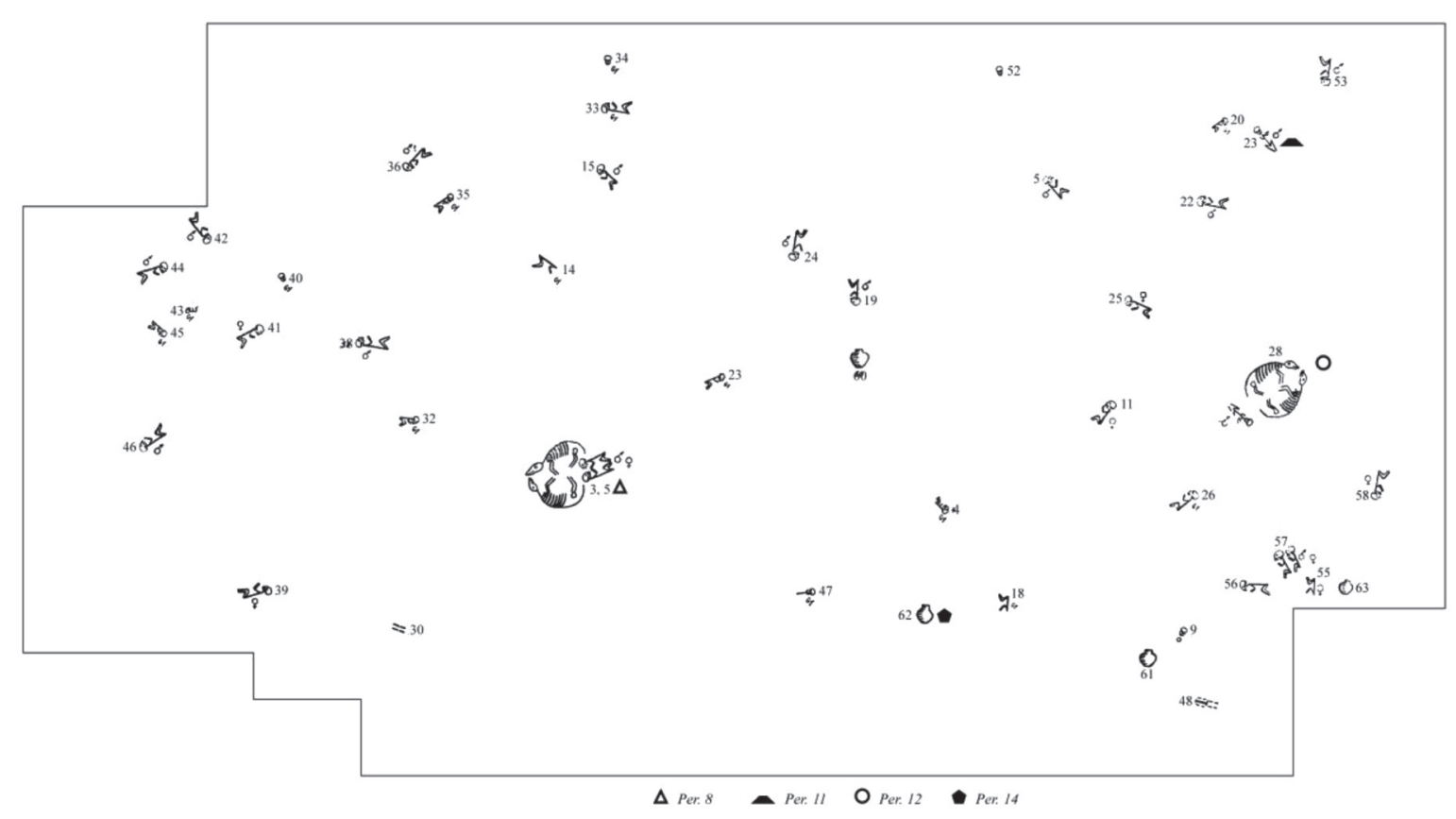

Gräberfeldsplan 5. Verteilung der durch mGruppen seriierten Bestattungen in Alsónémedi

Durch die Seriation gemäß Typen konnte die zeitliche Stellung nur im Fall eines Grabs von Alsónémedi bestimmt werden: Das Grab an22 vertritt die Periode 13.

Resümee. In Alsónémedi handelt es sich um die Gräber der klassischen Badener Kultur. Die Belegung des Gräberfeldes dürfte mit dem Doppelgrab 3/5 im zentralen Bereich angefangen haben. Die Belegung des Bestattungsplatzes scheint in der Seriation der Gräber durch mGruppen bis zum Ende bruchlos gewesen zu sein.

\subsection{Fonyód-Bézsenypuszta}

In diesem kleinen Gräberfeld findet man zwei Befunde, die gemäß mGruppen zeitlich zugeordnet werden konnten. Die Periode 7 vertritt das Grab fb4 mit einem Henkelbecher und einer profilierten Schüssel (Typen 1b3a3 und 2d2a1) und die Periode 11 das Grab fb2 mit zwei Henkelbechern und einer profilierten Schale (Typen 1b1c1, $1 \mathrm{~b} 5 \mathrm{~b} 8,2 \mathrm{f} 2 \mathrm{c} 2)$.

Resümee zu Fonyód-Bézsenypuszta. Die Keramik aus beiden, nur durch mGruppen seriierten Bestattungen von Fonyód-Bézsenypuszta weist die typologischen Merkmale der klassischen Badener Keramik auf. Die dreigliedrigen Henkelbecher des Typs 1b3a3 und die profilierte Schüssel des Typs 2d2a1 aus dem Grab 4 bzw. die Typen 1b5b8 von Henkelbechern und 2f2c2 von profilierten Schalen aus dem Grab 2 besitzen die Charakteristik der klassischen Badener Kultur.

\subsection{Balatonlelle-Felsö Gamász.}

An dieser Fundstelle gibt es 14 Bestattungen, die gemäß mGruppen zeitlich zugeordnet werden konnten. Sie vertreten insgesamt sechs Perioden (Diagramm 10).

7,14\% $(\mathrm{n}=1)$ der Befunde (Grab fg8) datiert in die Periode 1. Ein Grab (Grab fg10) stammt aus der Periode 8 und ein anderes ( $\mathrm{Grab} f \mathrm{fg} 7)$ aus der Periode 9. 21,43\% $(\mathrm{n}=3)$ der Bestattungen ist für die Periode 10 typisch (Grab fg5, fg15, fg20), während 14,29 \% ( $\mathrm{n}=2)$ von ihnen aus der Periode 11 stammt (Grab fg 16, fg22). Mit dem größten Prozentsatz, mit 42,86 \% ( $\mathrm{n}=6$ ), findet man hier Befunde der Periode 12 (Grab fg6, fg13, fg14, fg17, fg21, fg23). 
Die räumliche Verteilung der durch mGruppen seriierten Gräber zeigt Gräberfeldsplan 6.

Gemäß Typen wurde die chronologische Stellung von 12 Gräbern festgestellt. Sie stammen aus drei Perioden (Diagramm 11).

$8,33 \%(\mathrm{n}=1)$ der Gräber vertritt die Periode 1. Im Grab fg8 wurde eine Henkeltasse des Typs 2a4b1 mit einer profilierten Schale des Typs 2f1c1 vergesellschaftet. Diese Bestattung liegt an der südlichen Peripherie der Nekropole, von den anderen Gräbern entfernt.

Die jüngeren Gräber verteilen sich in drei größere Gruppen.

25,0 \% ( $\mathrm{n}=3)$ der Bestattungen gehört der Periode $13 \mathrm{zu}$. Das Grab fg7 (Typen 1b4c1, 2c1d5, 2f2c4) liegt in der südöstlichen Gräbergruppe. Das Grab fg17 in der südwestlichen Gräbergruppe weist sechs Typen auf (1b4b1, 1b4c1, 1b5c1, 1b5d1, 1b5d2, 1b6c1, 1c3a2, 2a3b2, 2b1a6, 2f2c4). Die Bestattung fg21 (Typen 1a4b3, 1b4d1, 1b5b6, 1b5d1, 2a3b2, 2a3b6, 2b1a5, 2f1b4) befindet sich dagegen in der nordwestlichen Gräbergruppe.

Das Vorkommen der durch Typen seriierten Bestattungen dieser Nekropole stellt Gräberfeldsplan 7 dar.

Die Mehrheit, 66,67 \% ( $\mathrm{n}=8)$ der Befunde stammt aus der Zeit der Periode 14. Auffallend ist, dass diese Bestattungen fast immer bei den Gräbern oder nahe den Bestattungen der vorigen Periode liegen. Nur das Grab fg5 (Typen 1b2c1, 1b4b3, 2f1b4) liegt weit nördlich von diesen. Das Grab fg6 der Periode 14 (Typen 1b3a8, 1b5b7,

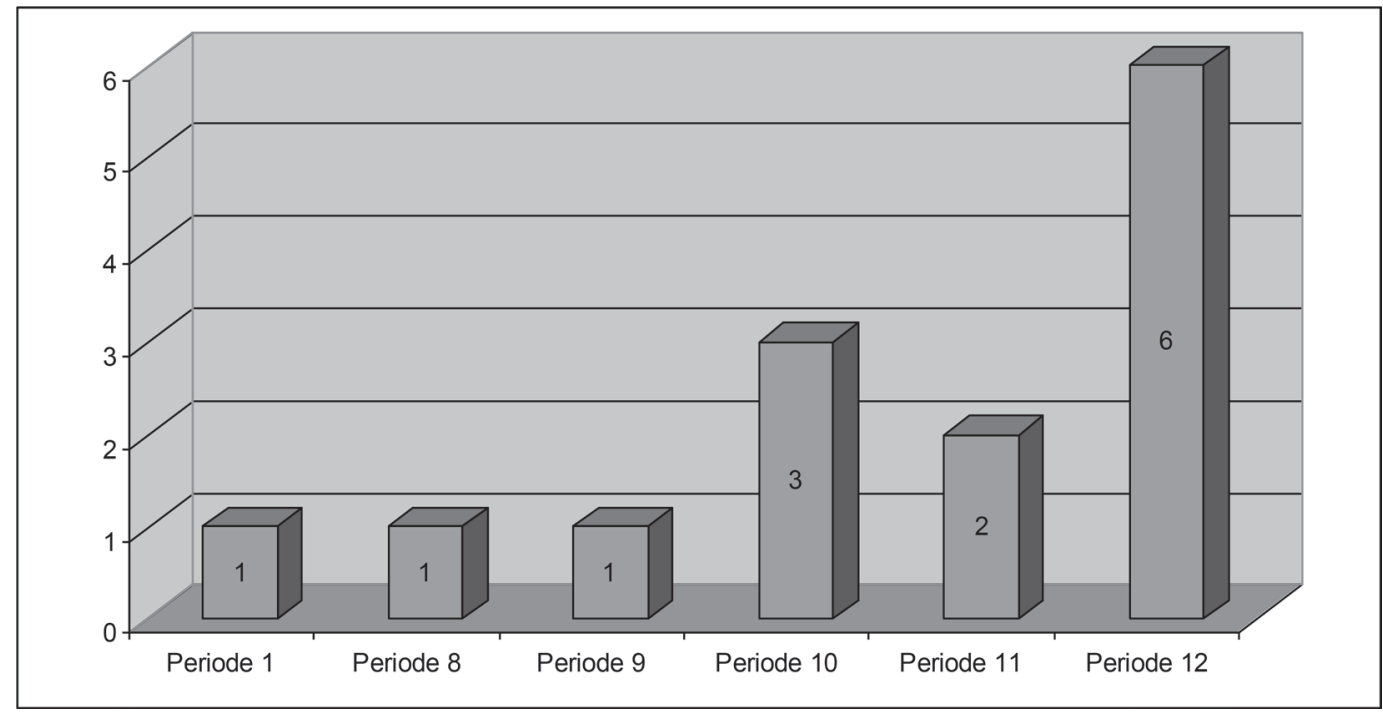

Diagramm 10. Verteilung der Gräber in Balatonlelle-Felső Gamász auf Grund der durch mGruppen definierten Perioden

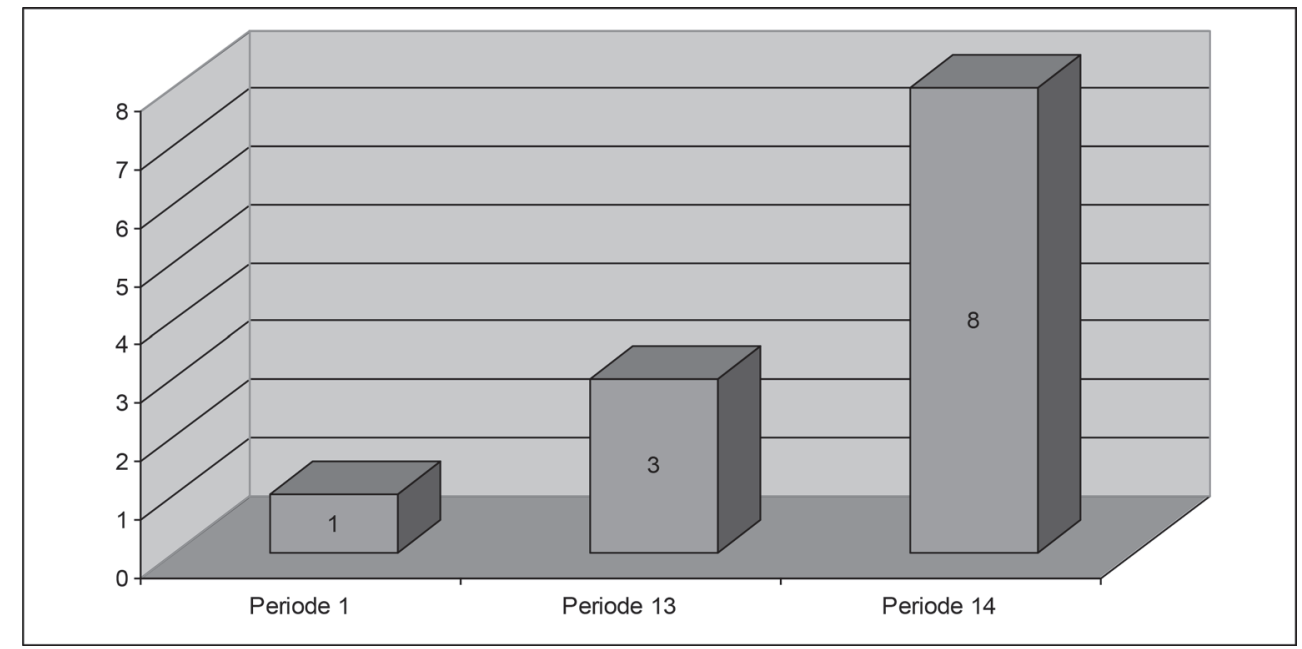

Diagramm 11. Verteilung der Gräber in Balatonlelle-Felső Gamász auf Grund der durch Typen definierten Perioden 
2f1b3) liegt bei dem erwähnten Grab fg7 der Periode 13. Das Grab fg13 (Typen 1a2a1, 1a4b3, 1b5c2, 1b5d2, 1c3a5, 1c5a2, 2f1b5, 2f2b1), das Grab fg14 (Typen 1b5c2, 1b5d5) bzw. das Grab fg17 (Typen 1b4b1, 1b4c1, 1b5c1, 1b5d1, 1b5d2, 1b6c1, 1c3a2, 2a3b2, 2b1a6, 2f2c4) kommen in der unmittelbaren Nähe der erwähnten Bestattung fg15 der Periode 13 vor.
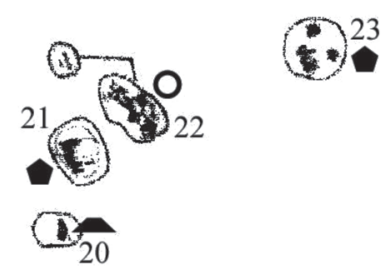

(1) 19

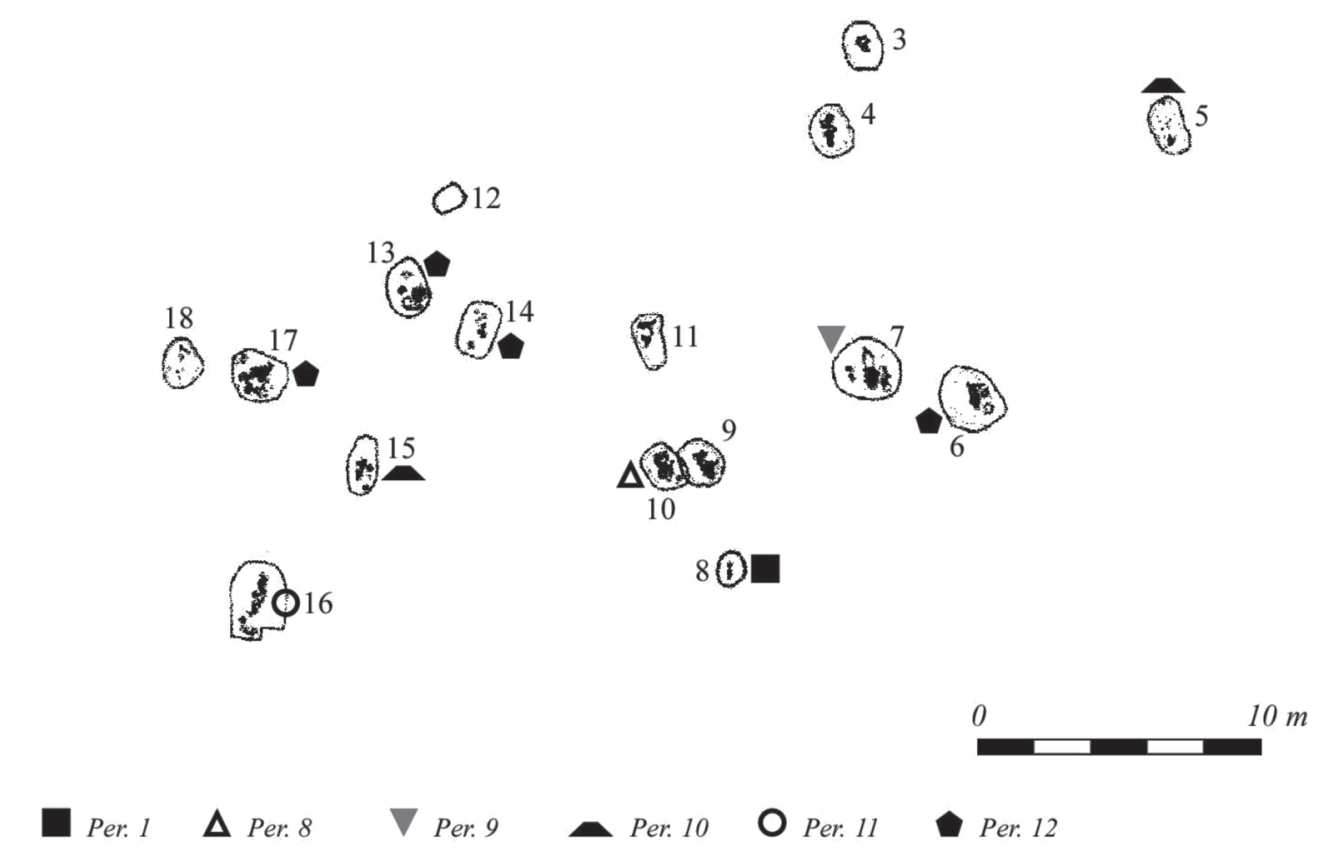

Gräberfeldsplan 6. Verteilung der durch mGruppen seriierten Bestattungen in Balatonlelle-Felső Gamász 
Ähnliches ist in der nordwestlichen Gräbergruppe zu beobachten, wo drei Bestattungen der Periode 14 neben dem älteren Grab fg21 bzw. ihm nahe liegen. Das Grab fg20 besaß die Typen 1b5c1, 1b5c2, 1d2a2, 2f1b5. Auch das Grab fg22 weist zahlreiche Gefäßtypen auf (1b3b3, 1b4c4, 1b5c1, 1b5d3, 1b6b1, 1b6c1, 1b6c2, 1d1a3, 2a4c1, 2f2b3). Im Grab fg23 konnten acht Gefäßtypen festgestellt werden (Typen 1b5d2, 1b6c1, 1b6c2, 1c3a3, 2b1a1, 2b1a6, 2f1b4, 2f2b3).
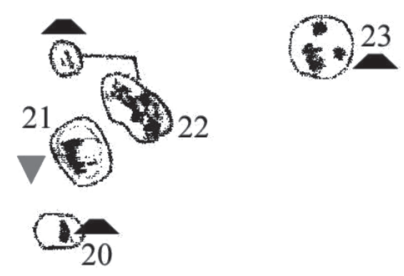

(19 19
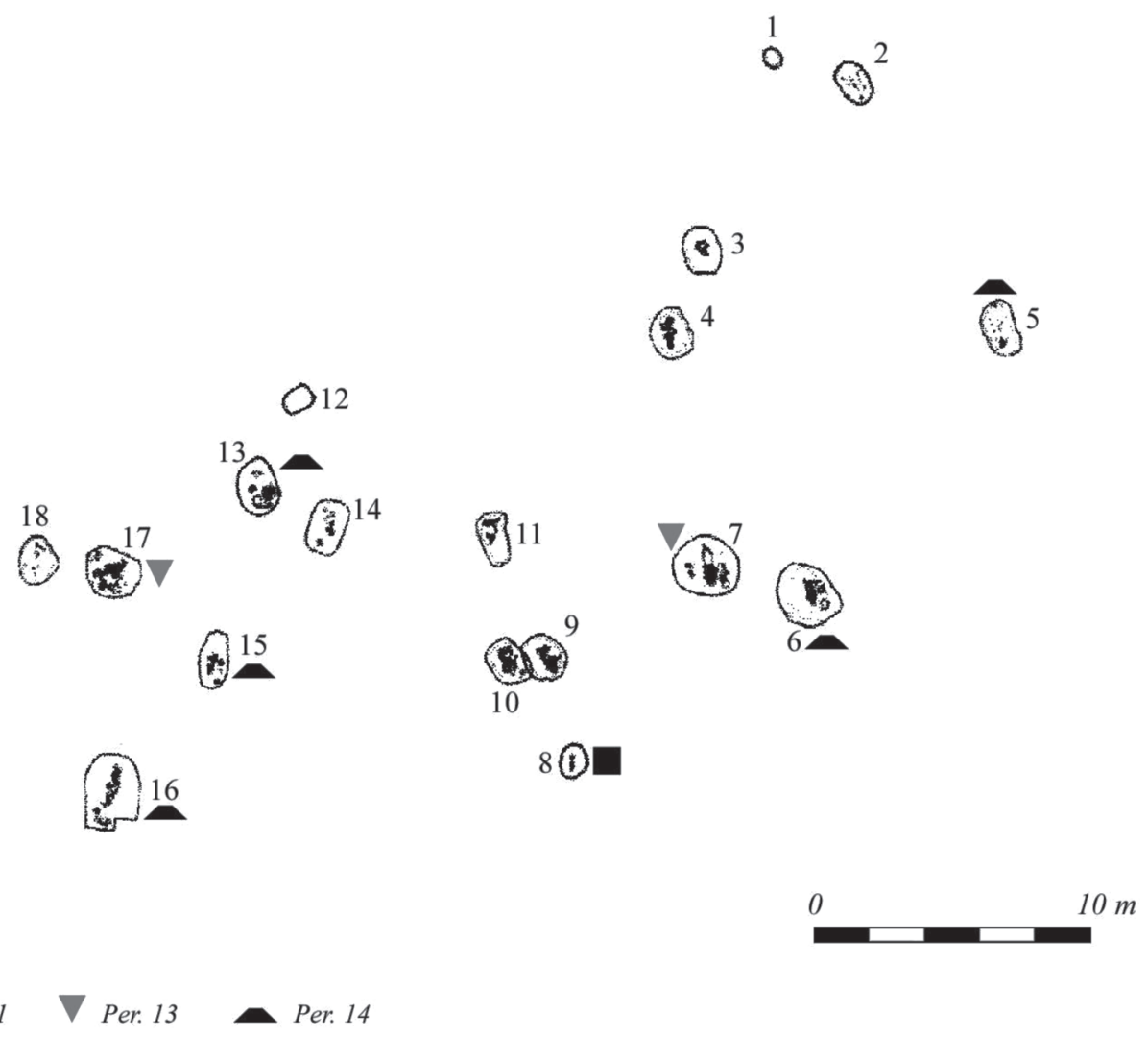

Gräberfeldsplan 7. Verteilung der Gräber in Balatonlelle-Felső Gamász auf Grund der durch Typen definierten Belegungsphasen 
Resümee zu Balatonlelle-Felső Gamász. Die Belegung des Bestattungsplatzes von Balatonlelle-Felső Gamász dürfte mit dem Grab fg8 an der südlichsten Peripherie der Nekropole begonnen haben. Durch die Analyse gemäß Typen vertritt dieses Grab die Periode 1. Parallelfunde der Henkeltasse 2a4b1 sind in den Bestattungen der Periode 1 von Pilismarót-Basaharc zu belegen. Auch die profilierte Schale der $m$ Gruppe 2f1c erscheint in Pilismarót, aber nicht nur in der Periode 1, sondern auch während der Perioden 3-10.

Alle anderen, durch Typen seriierten Gräber von Balatonlelle-Felső Gamász repräsentieren die Periode 13 und Periode 14, d. h. die klassische Badener Kultur. Ein zeitlicher Bruch in diesem Gräberfeld kann nur zwischen dem Grab fg8 der Periode 1 und den anderen der Perioden 13 und 14 angenommen werden.

\subsection{Mezöcsát-Hörcsögös}

Die relativchronologische Stellung durch Seriation gemäß mGruppen wurde im Fall von sechs Befunden aus fünf Perioden bestimmt (Diagramm 12).

Aus der Periode 8 (Grab mcs9), der Periode 10 (Grab mcs7), der Periode 12 (Grab mcs8) und der Periode 14 (Grab mcs8) stammt je eine Bestattung. 33,33\% $(\mathrm{n}=2)$ der Gräber datieren zugleich in die Periode 11 (Grab $\operatorname{mcs} 2$, mcs12).

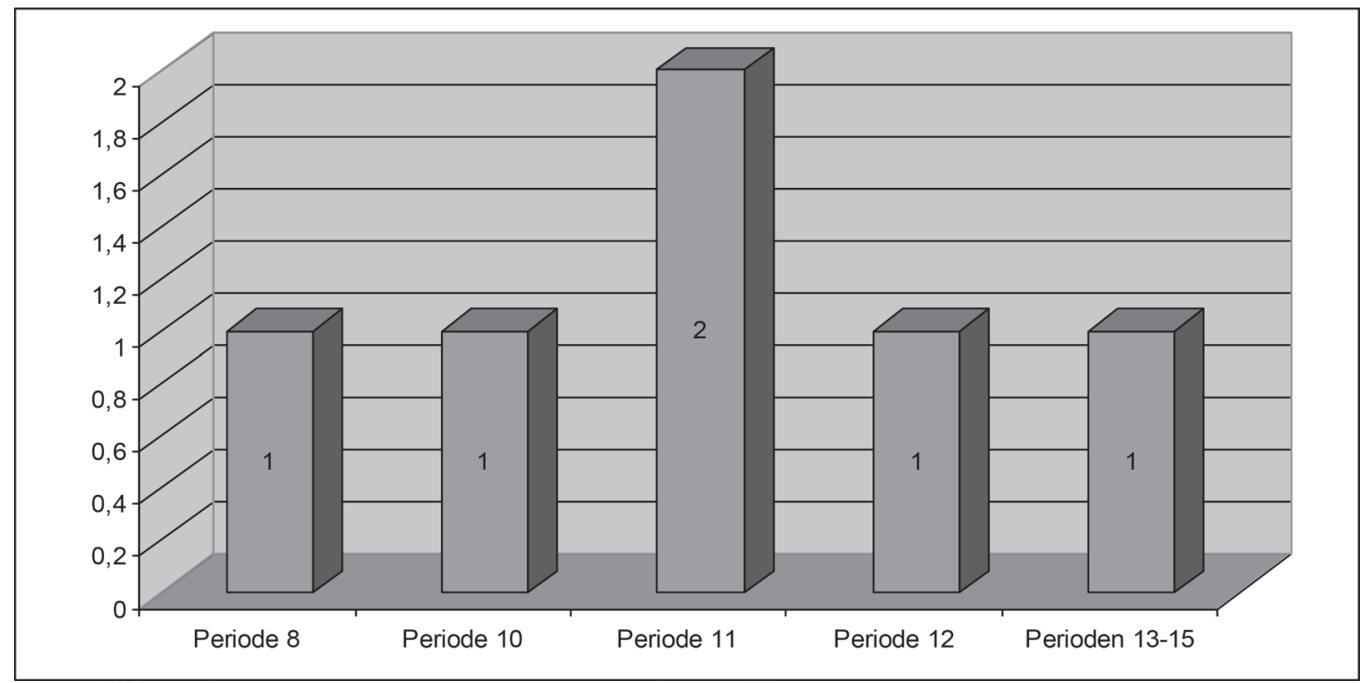

Diagramm 12. Verteilung der Gräber in Mezőcsát-Hörcsögös auf Grund der durch mGruppen definierten Perioden

Die räumliche Verteilung dieser Bestattungen im Gräberfeld stellt Gräberfeldsplan 8 dar. gramm 13).

Die zeitliche Stellung der Gräber durch Seriation von Typen wurde bei sechs Gräbern bestimmt (Dia-

Die Mehrheit, 83,33\% $(\mathrm{n}=5)$ der Befunde datiert in die Periode 13 (Grab mcs8, mcs6, mcs9, mcs12, mcs7) und nur einer von ihnen dürfte die Periode 14 vertreten (Grab mcs2). plan 9 vor.

Das Vorkommen der durch Typen seriierten Bestattungen in Mezőcsát-Hörcsögös stellt Gräberfelds-

Die Gräber der durch die Seriation der Typen bestimmten Periode 13 verteilen sich im mittleren und östlichen Bereich der Nekropole. Das Grab mcs9 (Typen 1b3b6,1c1b2, 1e1b1, 2a2a1, 2e1a3, 2f2c3) liegt am Nordrand, das Grab mcs7 (Typen 1b3b2, 1b4b6, 1c1b2, 1c2c1, 2f2b1, 2f2b2, 2f2c2, 2f2c3) östlich davon und die Gräber mcs8 (Typen $1 \mathrm{~b} 5 \mathrm{c} 4,1 \mathrm{c} 3 \mathrm{a} 2,2 \mathrm{a} 3 \mathrm{c} 4$ ) und mcs12 (Typen 1b4c1, 1b5b4, 2f2c2) südlich von dem Vorigen. Die Bestattung mcs6 (Typen 1b4b3, 1b5c4, 2a2a3) kam an der südlichen Peripherie des Gräberfeldes zum Vorschein.

Das die Periode 14 vertretende Grab mcs2 (Typen 1b4b6, 1e2a1) befindet sich am südöstlichen Rand der Nekropole zwischen den Bestattungen mcs8 und mcs12 der vorigen Periode. 


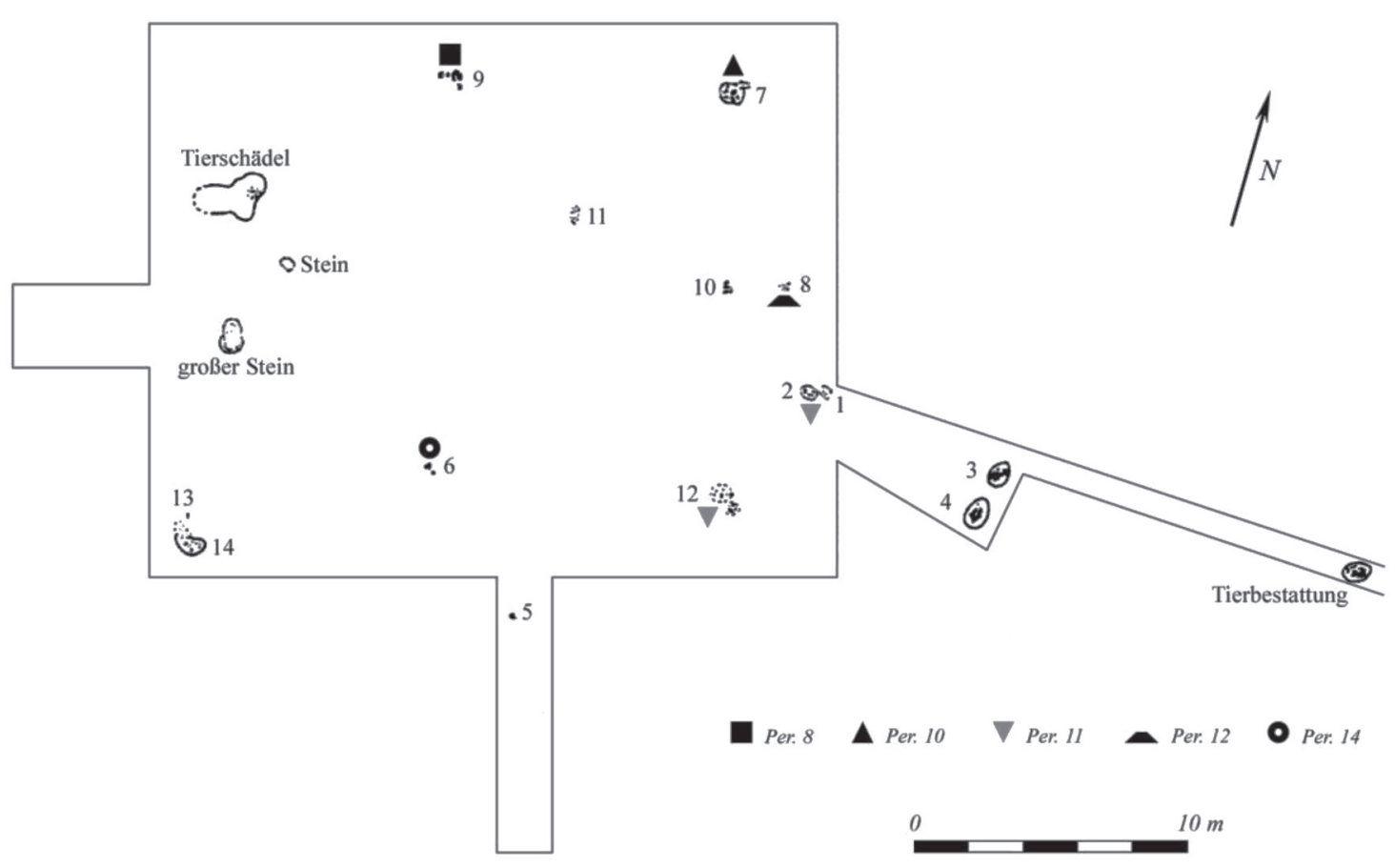

Gräberfeldsplan 8. Verteilung der Gräber in Mezőcsát-Hörcsögös auf Grund der durch mGruppen definierten Belegungsphasen

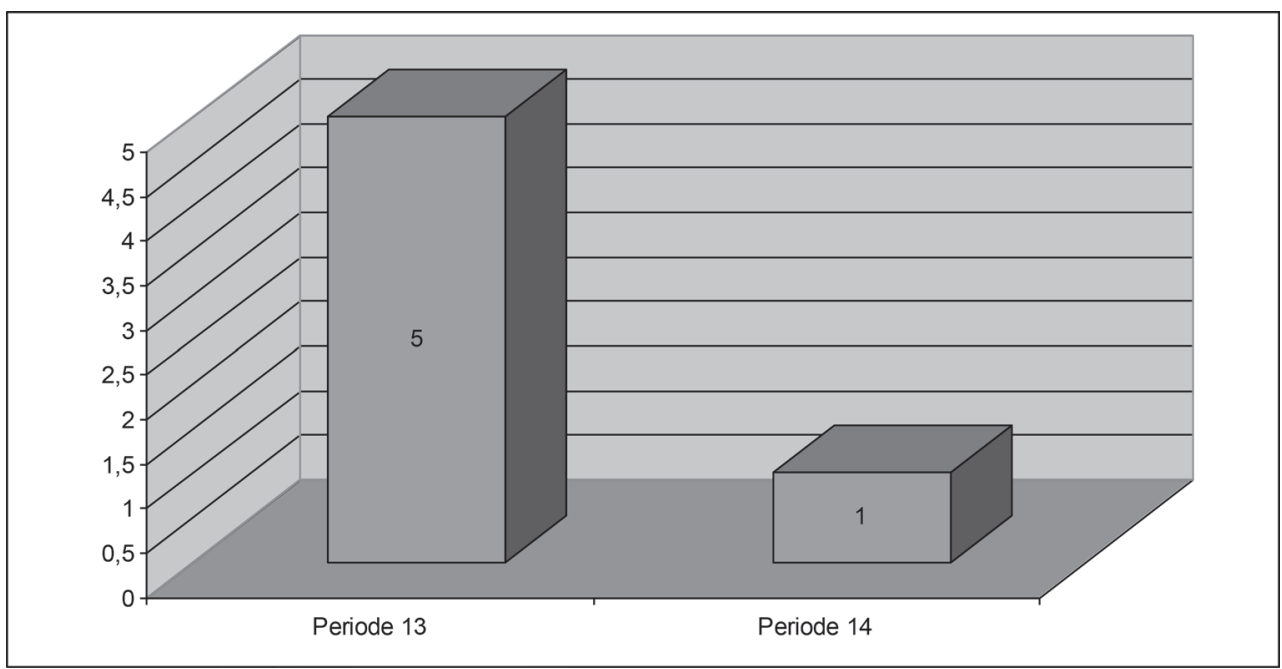

Diagramm 13. Verteilung der Gräber in Mezőcsát-Hörcsögös auf Grund der durch Typen definierten Perioden

Resümee zu Mezőcsát-Hörcsögös. Der keramische Bestand der Gräber von Mezőcsát-Hörcsögös weist einen einheitlichen typologischen Charakter auf. Die Ergebnisse der metrischen Analyse deuten darauf hin, dass diese Nekropole zur Zeit der klassischen Badener Kultur belegt wurde. Wie gesehen, gehört die Mehrheit der durch Typen seriierten Bestattungen der Periode 13 an und Grab 2 aus der Periode 14 dürfte hier als der jüngste Befund behandelt werden. 


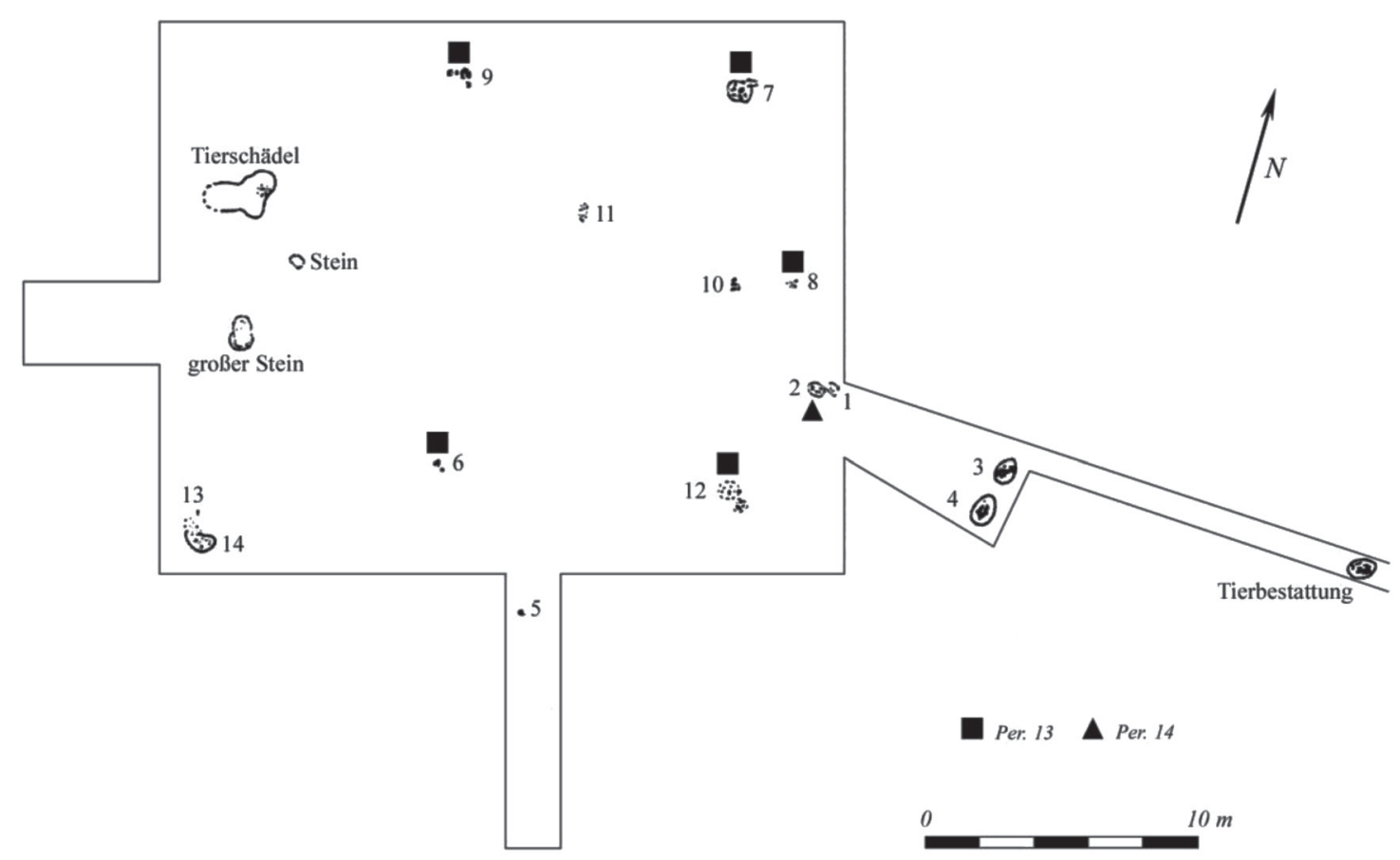

Gräberfeldsplan 9. Verteilung der Gräber in Mezőcsát-Hörcsögös auf Grund der durch Typen definierten Belegungsphasen

6. ERGEBNISSE

1. Es wurde jüngst die typochronologische Analyse der Grabkeramik von zwei Kulturen oder Kulturkreisen vorgenommen.

Lengyel-Kultur. Die Gefäßkeramik dieser spätneolithisch-frühkupferzeitlichen Gemeinschaften weist in ihrem Verbreitungsgebiet, besonders im Karpatenbecken, während ihrer ganzen Entwicklungszeit gemäß Form und Verzierung nur wenige Änderungen auf. ${ }^{88}$

Polgár-Komplex. Bei der Analyse der Grabkeramik des Polgár-Komplexes tauchte das Problem auf, dass die Gemeinschaften mit Tiszapolgár- und die mit Bodrogkeresztúr-Keramik nahezu zeitgleich miteinander waren, nebeneinander lebten und sich einander nahe bestatteten. Gräber beider Gemeinschaften sind in beträchtlicher Anzahl nur im Gräberfeld von Basatanya vorhanden. Im Fall des Polgár-Komplexes handelt es sich also um zwei fast zeitgleiche Gemeinschaften mit unterschiedlichen „Keramikstilen“. ${ }^{89}$

Im Fall der vorliegenden Analyse der Keramik der Bandener Kultur handelt es sich nur um sechs Nekropolen einer prähistorischen Kultur, deren Grabkeramik zugleich zwei, in Form und Verzierung abweichende Stile (Boleráz und klassisches Baden) aufweist. Die untersuchten Gräberfelder befinden sich in weit voneinander liegenden Regionen des Karpatenbeckens.

Die Grabkeramik der Badener Gemeinschaften bearbeiteten wir durch die Anwendung der metrischen Analyse und Seriation für die Festlegung der zeitlichen Kontexte, und zwar unabhängig von den früheren Ergebnissen der Bayesischen und 14C-Untersuchungen. Wir sind der Meinung, dass die Analysen durch naturwissenschaftliche Methoden die vorherigen Ergebnisse der keramischen Merkmalanalyse oder metrischen Analyse nicht verpassen dürfen.

Die keramische Typologie wurde dazu in einem hierarchischen System aufgebaut, welches im Grunde genommen der biologischen Taxonomie entspricht. Die metrische Analyse dieser Grabkeramik beruht auf zwei 
Arten der Gruppierung der Funde und Befunde, nachdem wir die Funde sowie die Befunde einerseits durch metrische Gruppen (mGruppen), andererseits durch Typen gruppierten. Die Bestimmung der Belegungsphasen durch mGruppen ergab eine Sequenz von 119 Gräbern und durch die Typen eine andere Sequenz von 73 Gräbern. Die Feststellung der 15 Perioden durch mGruppen führte zu einer Sequenz von 107 Gräbern und durch Typen zur Reihenfolge von 75 Gräbern. Wir möchten hier wiederholen, dass es sich im Fall der mGruppen um viel größere typologische Einheiten der Grabkeramik handelt, während die Analyse durch Typen wesentlich kleinere keramische Gruppen ergab.

Auf die typologische Gruppierung der untersuchten Keramikgefäße folgten die Analyse der relativchronologischen Lage der Gräber mit diesen Funden und die Bestimmung der chronologischen Entwicklung der Nekropolen. Als erster Schritt wurden drei Belegungshorizonte (Früh-, Übergangs- und Späthorizont) bestimmt.

Das Seriationsprogramm ermöglichte es, höchstens 15 typochronologische Einheiten innerhalb des untersuchten Grabbestandes erfassen zu können. Auf diese Weise, in einem zweiten Schritt haben wir deshalb 15 Perioden (künstliche Perioden) bestimmt.

2. Gestützt auf das Vorkommen der an mehreren Nekropolen vorhandenen Typen stellt Diagramm 14 die möglichen Zusammenhänge zwischen den diskutierten Nekropolen dar: 209 von 264 Typen treten nur auf den jeweiligen Fundplätzen auf. 32,54 \% ( $\mathrm{n}=68)$ dieser Typen kommen nur in Pilismarót vor und fast die Hälfte, 44,02 \% $(\mathrm{n}=92)$ von ihnen erscheint nur in Budakalász. 13,88 \% $(\mathrm{n}=29)$ dieser Typen konnte nur in Balatonlelle, 4,78\% $(\mathrm{n}=10)$ nur in Alsónémedi, 1,44 \% $(\mathrm{n}=3)$ nur in Fonyód und 3,35\% $(\mathrm{n}=7)$ nur in Mezőcsát nachgewiesen werden. Diese Daten sind selbstverständlich auch von der Anzahl der ausgegrabenen Befunde und Funde pro Nekropole abhängig.

Die gegenseitigen Beziehungen zwischen den diskutierten Nekropolen studieren wir anhand der in mehreren Nekropolen nachgewiesenen Gefäßtypen (Diagramm 14).

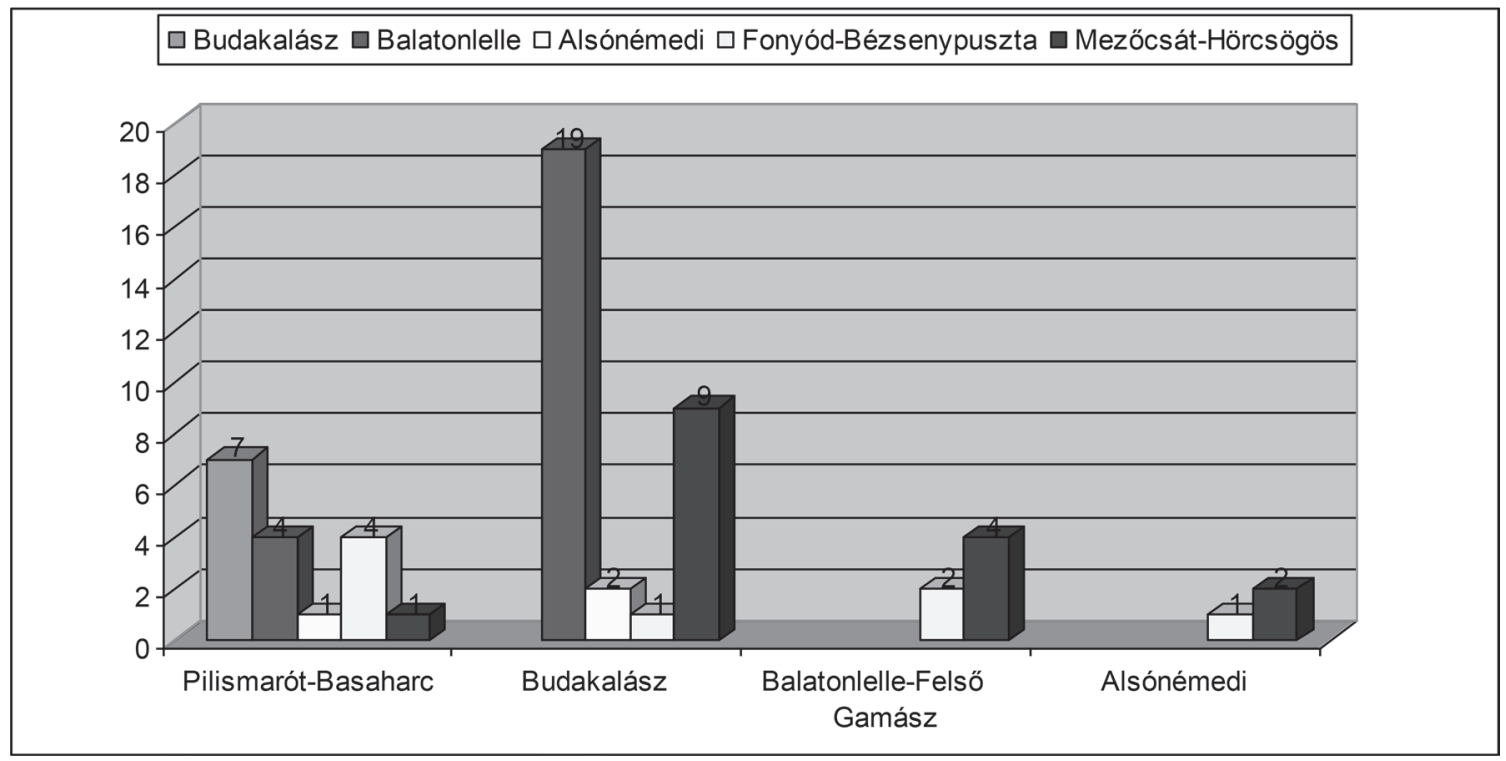

Diagramm 14. Zusammenhänge zwischen den Nekropolen auf Grund der keramischen Typen

Pilismarót-Basaharc. 41,18 \% $(\mathrm{n}=7)$ solcher Typen erscheint hier und auch in Budakalász (Typen 1b1a1, 1c1b1, 1d1b4, 2d2a3, 2a4a1, 2f1a3, 2d2a1). Zwischen Pilismarót-Basaharc und Balatonlelle-Felső Gamász (Typen 1d1a3, 2a4b1, 2c1d5, 2f1c1) bzw. Fonyód-Bézsenypuszta (Typen 2d2a1, 1b2b1, 1d1a2, 2d2a4) nimmt der Anteil der gemeinsamen Typen je 23,53 \% ( $n=4-4)$ ein. In Alsónémedi (Typ 1d2a3) und Mezőcsát-Hörcsögös (Typ 1c2c1) findet man nur je einen Typ, der auch in Pilismarót auftritt.

Budakalász. Beachtenswert ist, dass 61,29\% $(\mathrm{n}=19)$ der hier vorhandenen Typen auch in Balatonlelle-Felső Gamász vorkommt (Typen 1a2a1, 1b1a4, 1b1c2, 1b3a8, 1b5b6, 1b6b1, 1b6b2, 1b6b3, 2a4c1, 2b1a1, 1c3a4, 1c3a5, 1c4a3, 2b1a5, 2f1b1, 2f1b4, 2f2c4, 2a3b2, 1c3a2). In Mezöcsát-Hörcsögös sind solche Typen mit 29,03 \% (n =9) 
belegt (Typen, 1c3a2, 1a3c1, 1b3b2, 1b3b6, 1b5c3, 1e2a1, 2a2a1, 2a3c4, 2e1a3, 2f2c1). In Alsónémedi befinden sich zwei solche Typen (2a3b2, 1b3a7), in Fonyód-Bézsenypuszta ist dagegen nur ein solcher Typ bekannt (Typ 1b3a3).

Balatonlelle-Felső Gamász. Der Anteil der hier und in Fonyód-Bézsenypuszta nachgewiesenen gemeinsamen Typen beträgt nur 33,33 \% $(\mathrm{n}=2)$ (Typen 1b1b3, 1b5b7), in Mezőcsát-Hörcsögös dagegen 66,67 \% $(\mathrm{n}=4)$ (Typen 1b4b3, 1b4c1, 1b5b4, 2f2b1).

Alsónémedi. Ein gemeinsamer Typ erscheint nur in Fonyód-Bézsenypuszta (Typ 2f2c2) und eine andere kann auch in Mezőcsát-Hörcsögös bestätigt werden (Typ 1c1b2).

3. Auf Grund der 14C-Untersuchungen behandelt E. M. Wild die „Stúrovo-Protoboleráz-Gruppe“ als Baden Ia. Seine Radiokarbondaten zeigt Abbildung 6. Die Boleráz-Gruppe dürfte die Ib-Ic-IIa-Phasen der Badener Kultur vertreten. ${ }^{90}$ Man kann E. M. Wild und Zs. Siklósi zustimmen: Die klassische Badener Periode kann von der Protoboleráz- und Boleráz-Periode klar getrennt und auf Grund der Radiokarbondaten in die Zeit von 3350 (68,2 \%) bis 2870 BC datiert werden. ${ }^{91}$

\begin{tabular}{|c|c|c|c|c|}
\hline Group name & $\begin{array}{l}\text { Nr of } \\
\text { samples }\end{array}$ & $\begin{array}{l}\text { Baden Culture } \\
\text { phase }\end{array}$ & $\begin{array}{l}68.2 \% \text { - time interval } \\
\text { of sum calibration }\end{array}$ & $\begin{array}{l}95.4 \% \text { - time interval. } \\
\text { of sum calibration }\end{array}$ \\
\hline $\begin{array}{l}\text { Sturovo- } \\
\text { Protoboleraz }\end{array}$ & 7 & Ia & $\begin{array}{l}3630 \mathrm{BC}(14.1 \%) 3580 \mathrm{BC} \\
3540 \mathrm{BC}(54.1 \%) 3360 \mathrm{BC}\end{array}$ & $\begin{array}{l}3750 \mathrm{BC}(94.3 \%) 3300 \mathrm{BC} \\
3250 \mathrm{BC}(1.1 \%) 3100 \mathrm{BC}\end{array}$ \\
\hline Boleraz & 27 & Ib-Ic-IIa & $\begin{array}{l}3640 \mathrm{BC}(46.0 \%) 3490 \mathrm{BC} \\
3470 \mathrm{BC}(4.2 \%) 3450 \mathrm{BC} \\
3440 \mathrm{BC}(17.9 \%) 3370 \mathrm{BC}\end{array}$ & $3700 \mathrm{BC}(95.4 \%) 3350 \mathrm{BC}$ \\
\hline
\end{tabular}

Abb. 6. Radiokarbondaten für die Protoboleráz- und Boleráz-Perioden (Wild et al. 2001, Tab. 2)

Die frühere Zeitstufe, d. h. die Boleráz-Gruppe der spätkupferzeitlichen Badener Kultur, tritt imKeramikmaterial sehr einheitlich, im ganzen Gebiet auf. ${ }^{92}$ Die Keramik der Badener Kultur weist während ihrer Entwicklungsphasen dagegen unterschiedliche Charakterzüge auf. ${ }^{93}$ Dieses Phänomen zeichnet sich in erster Linie zwischen der Grabkeramik von Pilismarót-Basaharc (Boleráz) und der anderen untersuchten Nekropolen der Badener Kultur (klassisches und spätes Baden).

Wir stimmen auch M. Furholt zu, dass die folgende Periode (3350-3100 BC), was die Gefäßkeramik angeht, durch eine deutliche Regionalisierung gekennzeichnet werden kann, ,indem sich verschiedene Stile der klassischen Badener Keramik bzw. des sog. Post-Boleráz in den verschiedenen Regionen voneinander unterscheiden lassen“. ${ }^{94}$ Wie auch die Ergebnisse unserer Analysen beweisen, zeigt sich eine starke Regionalisierung während der „klassischen Stufe“ der Badener Kultur. ${ }^{95}$

Auf Grund der von Pilismarót-Basaharc und Budakalász stammenden Radiokarbondaten können wir drei Belegungsphasen innerhalb der Entwicklung der Badener Kultur bestimmen. In die Belegungsphase 1 kann die Mehrheit der Bestattungen von Pilismarót-Basaharc datiert werden. Die Belegungsphase 2 dürften einige Gräber von beiden Nekropolen vertreten, während die Gräber der Belegungsphase 3 in Budakalász nachgewiesen sind. Grundsätzlich dürfte es um zwei Zeiträume in diesem Fall gehandelt haben (Tabelle 9).

Die Radiokarbondaten aus Südosteuropa weisen darauf hin, dass das Gräberfeld von Budakalász mit den Kulturen Ezero XIII-VII und Sitagroi IV zeitgleich gewesen sein dürfte. ${ }^{96}$ Für die Belegungsspanne des Gräberfeldes hält Zs. Siklósi auf Grund der Ergebnisse ihrer Bayesischen Analyse 100-240 Jahre für wahrscheinlich. ${ }^{97}$

${ }^{90}$ WILD et al. 2001, 1062, Tab. 2.

${ }^{91}$ WiLd et al. 2001, 1062; SiKLÓSi 2009, 462.

${ }^{92}$ SACHßE 2009, 157.

93 „In the early phase (3650-3350 BC) coarse fabrics are regionally diverse and local in their context and meaning. The earliest fine wares, the Boleráz wares, have their first use in Austria (and the adjacent region), but then spread over a short time span to north and west mixing with other cultural attributes. This expansion, dated by new radiocarbon dates to $3520-3350 \mathrm{BC}$ coincides closely to the spread of wheeled vehicles as currently known“ (FURHOLT 2008a, 627). ${ }^{94}$ Furholt 2011, 255.
95 „Daraus resultiert eine Verselbstständigung einzelner Gebiete oder Überlagerung durch benachbarte Kulturen, soweit die Weiterentwicklung überhaupt verfolgt werden kann. Entsprechende regionale Entwicklungen sind auch in den Bestattungen der Badener Kultur vor allem rund um das Donauknie in Nordtransdanubien und der Südwestslowakei, im südlichen Transdanubien, in Niederösterreich, Mähren sowie im nördlichen Alföld erkennbar“" (SACHßE 2005; SACHßE 2008; SACHßE 2009, 157).

${ }^{96}$ WiLD et al. 2001, 1062-1063; SIKLÓSI 2009, 465.

${ }^{97}$ SiKLÓsi 2009, 465. 
Tabelle 9. Belegungsphasen in Pilismarót-Basatanya und Budakalász im Verhältnis zu den Radiokarbondaten

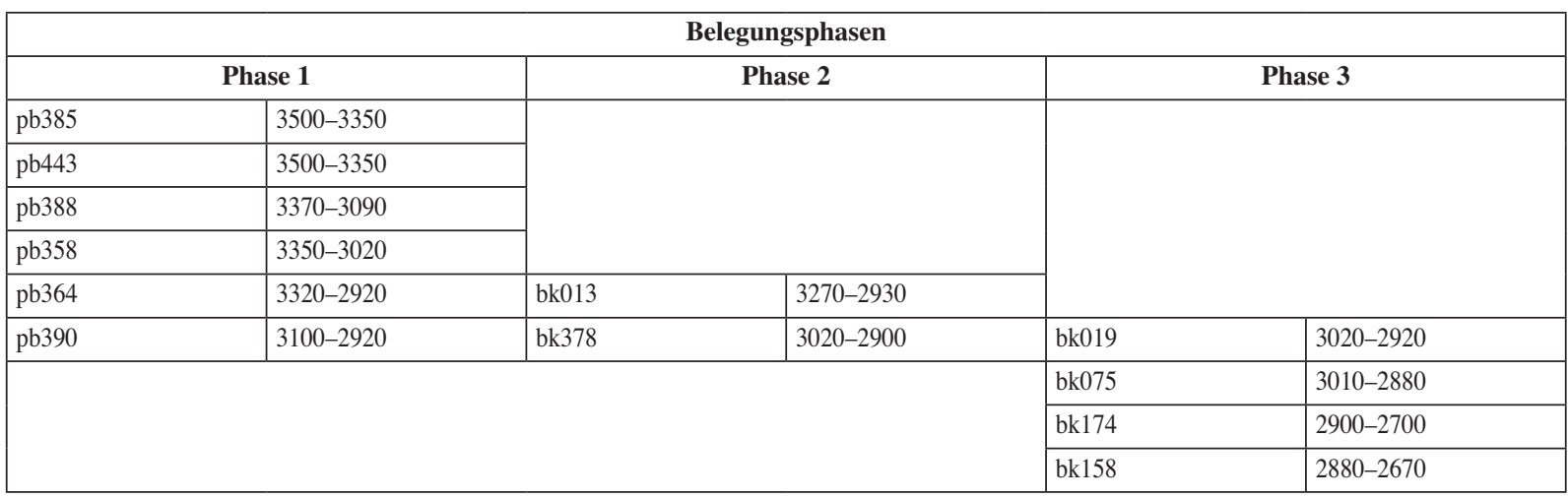

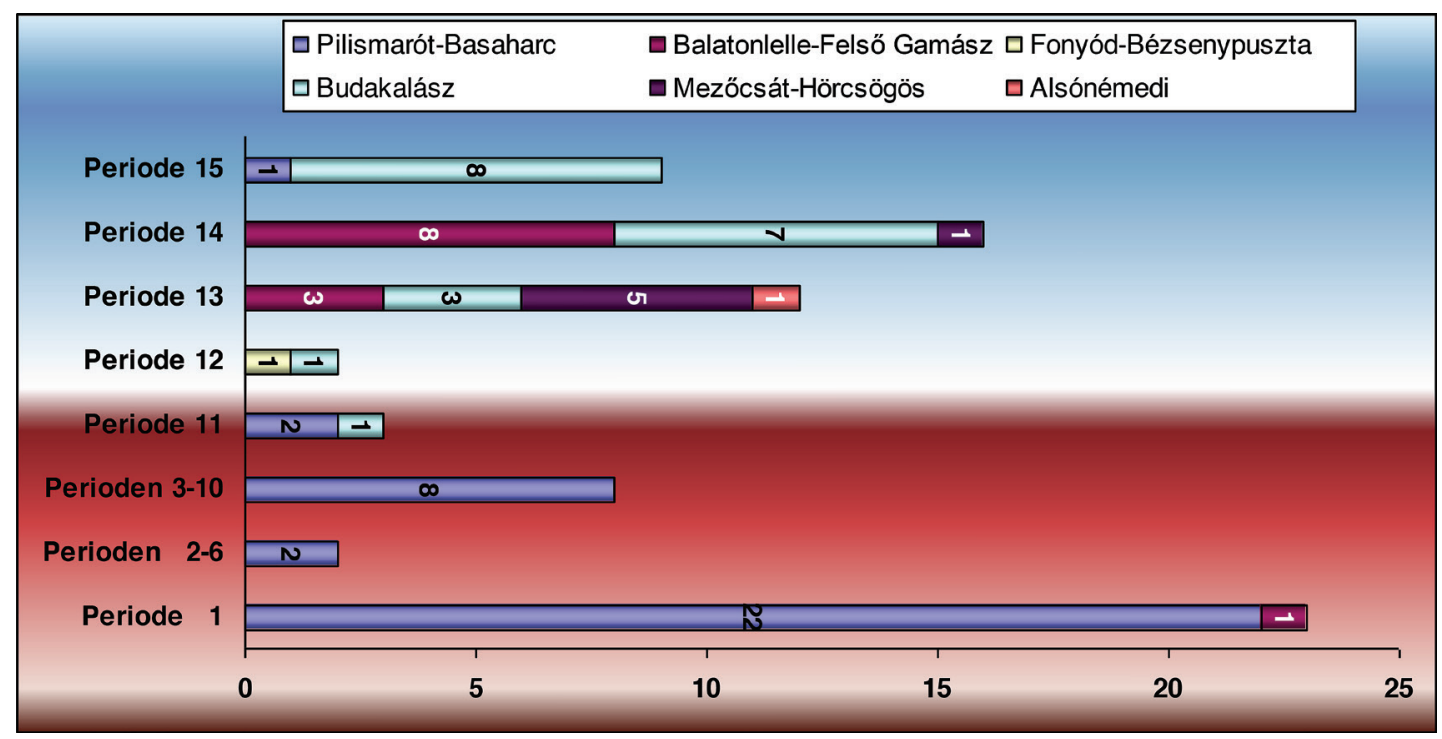

Diagramm15. Belegungsgeschichte der untersuchten Gräberfelder der Badener Kultur auf Grund der durch Typen definierten Perioden

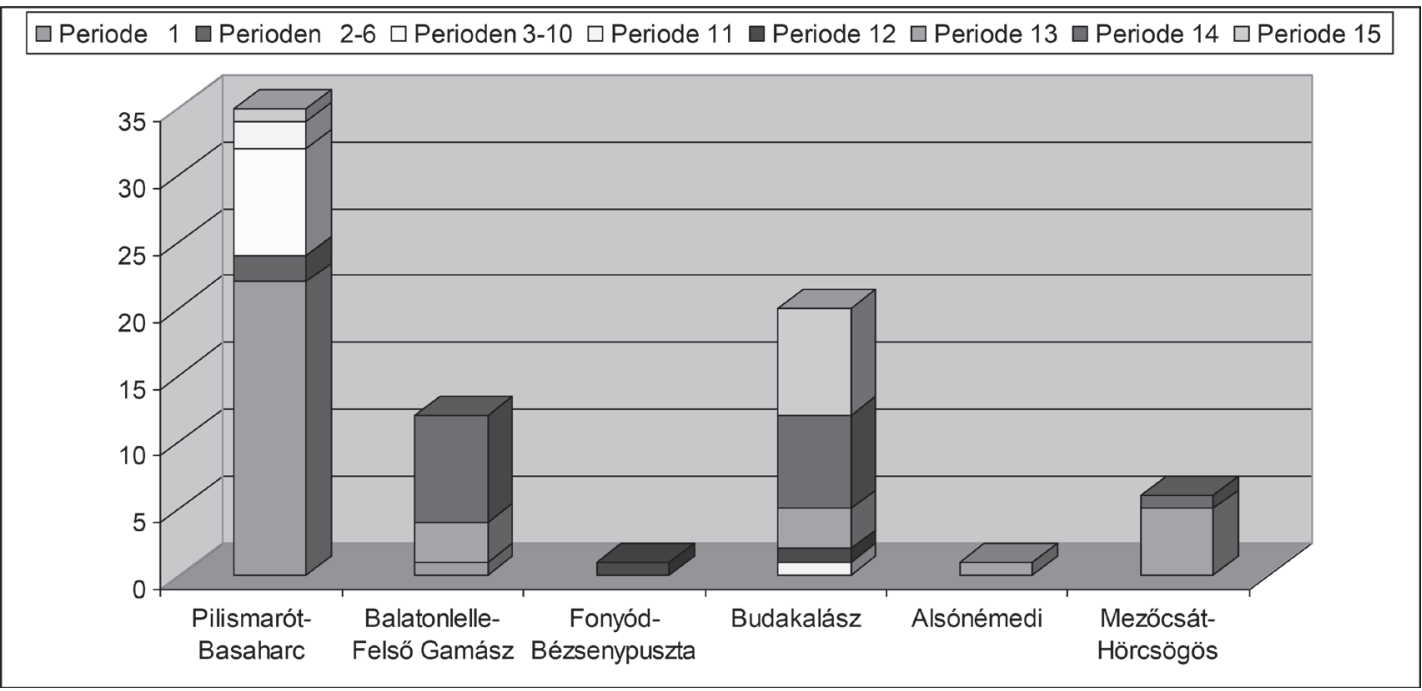

Diagramm 16. Belegungsgeschichte der untersuchten Gräberfelder der Badener Kultur auf Grund der durch Typen definierten Perioden 
Tabelle 10. Zeitliche Einordnung der Gräber von Pilismarót-Basaharc gemäß Ergebnisse von M. Bondár (2015, Tabl. 10)

\begin{tabular}{|c|c|c|}
\hline Horizon I & Horizon II & Horizon III \\
\hline 3645-3525 & $3500-3360$ & $3330-2920$ \\
\hline \multicolumn{3}{|c|}{$3500-3360-3330$ cal BC } \\
\hline Graves & Graves & Graves \\
\hline 337 & 336 & \\
\hline 340 & 338 & \\
\hline 341 & 339 & \\
\hline 343 & 346 & 353 \\
\hline 344 & 354 & \\
\hline 347 & 358 & \\
\hline 348 & 359 & \\
\hline 349 & 360 & \\
\hline 350 & 362 & \\
\hline 351 & 382 & 364 \\
\hline 352 & 385 & \\
\hline 355 & 386 & \\
\hline 356 & 387 & 388 \\
\hline 357 & $390 / a$ & \\
\hline 361 & 390 & \\
\hline 363 & 391 & 397 \\
\hline 365 & 392 & \\
\hline 383 & 393 & \\
\hline 384 & 395 & \\
\hline $384 / \mathrm{a}$ & 396 & \\
\hline 394 & 398 & \\
\hline 400 & 402 & 399 \\
\hline 401 & 403 & \\
\hline 407 & 405 & \\
\hline 408 & 406 & \\
\hline 412 & 409 & \\
\hline 411 & 413 & \\
\hline 419 & 414 & \\
\hline
\end{tabular}

\begin{tabular}{|c|c|c|}
\hline Horizon I & Horizon II & Horizon III \\
\hline $3645-3525$ & $3500-3360$ & $3330-2920$ \\
\hline \multicolumn{3}{|c|}{$3500-3360-3330$ cal BC } \\
\hline Graves & Graves & Graves \\
\hline 421 & 415 & \\
\hline 432 & 416 & \\
\hline 437 & 418 & \\
\hline 434 & 420 & \\
\hline 441 & 422 & \\
\hline 442 & 423 & \\
\hline 452 & 424 & \\
\hline 455 & 426 & \\
\hline & 427 & \\
\hline & 428 & \\
\hline & 433 & 429 \\
\hline & 435 & \\
\hline & 436 & \\
\hline & 439 & \\
\hline & 440 & \\
\hline & 443 & \\
\hline & 444 & \\
\hline & 445 & \\
\hline & 447 & \\
\hline & 448 & \\
\hline & 449 & \\
\hline & 450 & \\
\hline & 451 & \\
\hline & 453 & \\
\hline & 454 & \\
\hline & 456 & \\
\hline & 457 & \\
\hline & 458 & \\
\hline
\end{tabular}

4. Eine Periodisierung der Gräber der untersuchten spätkupferzeitlichen Nekropolen des Karpatenbeckens zeigen wir auf Grund der Ergebnisse der metrischen Analyse und der Seriation durch Typen im Verhältnis von 15 Perioden bei 75 Bestattungen vor (Diagramm 15).

Während der Perioden 1 bis 10 konnten Gräber nur aus Pilismarót-Basaharc bzw. einmal aus BalatonlelleFelső Gamász nachgewiesen werden. Diese Zeit könnte als Phase Baden 1a (Boleráz 1) definiert werden.

Die Periode 11 wird durch einige Befunde von Pilismarót-Basaharc und Budakalász charakterisiert. Es dürfte sich dabei um die Phase Baden $1 \mathrm{~b}$ handeln.

Während der Periode 12 erscheint je ein Grab aus Fonyód-Bézsenypuszta und Budakalász. Trotz der kleinen Gräberanzahl könnte diese Periode als Phase Baden 2a bezeichnet werden.

Die Gräber der Periode 13 (Balatonlelle-Felső Gamász, Budakalász, Mezőcsát-Hörcsögös, Alsónémedi) und Periode 14 (Balatonlelle-Felső Gamász, Budakalász, Mezőcsát-Hörcsögös) können zeitlich der Phase Baden $2 \mathrm{~b}$ (klassisches Baden) zugeteilt werden. 
Die Bestattungen der Periode 15 (Budakalász und einmal Pilismarót-Basaharc) wurden in Budakalász und einmal in Pilismarót-Basaharc festgestellt. Hierbei dürfte es sich um die Phase Baden 2c (Spätbaden) gehandelt haben. Beachtenswert ist, dass keine Befunde zwischen der Periode 11 und der Periode 14 in Pilismarót-Basaharc belegt werden können.

5. Die Belegungsgeschichte der untersuchten Gräberfelder können wir auf folgende Weise interpretieren (Diagramm 16).

Im Gräberfeld von Pilismarót dominieren die Gräber der Phase Baden 1a und 1b (Boleráz). Die chronologische Lage des Grabs der Phase Baden 2c bleibt hier weiterhin fraglich.

Die Ergebnisse der in der vorliegenden Arbeit vorgenommenen keramischen Analyse stimmen im Fall des Gräberfeldes von Pilismarót-Basaharc mit den früheren chronologischen Feststellungen von M. Bondár im Wesentlichen überein (Tabelle 10).

In Balatonlelle-Felső Gamász beginnt die Belegung der Nekropole während der Phase Baden 1a, aber die Mehrheit der Befunde vertritt die Phase Baden $2 b$.

Im Gräberfeld von Fonyód-Bézsenypuszta ist die chronologische Lage nur im Fall eines Grabs belegt: Es gehört in die Phase Baden 2a.

In Budakalász dürfte die Belegung des Bestattungsplatzes während der Phase Baden 1b angefangen haben und die Belegung des Gräberfeldes dürfte auch während der Phase Baden 2a, Phase 2b und Phase 2c gedauert haben.

Die einzige chronologisch bestimmte Bestattung von Alsónémedi repräsentiert die Phase Baden $2 \mathrm{~b}$.

Alle Gräber der bekannten Fälle von Mezőcsát-Hörcsögös wurden in die Phase Baden $2 b$ eingeordnet.

Bessere und detailliertere Ergebnisse sind in Bezug auf die Chronologie der Badener Kultur im Zuge der Analyse aller bekannten Gräberfelder zu erwarten.

\section{LITERATUR}

BANNER 1956

BIEHL-GLESER 2003

BONDÁR 1987

BONDÁR 1990-1991

BONDÁR 2002

BONDÁR 2009a

BONDÁR 2009b

BONDÁR 2009c

BONDÁR 2009d

BONDÁr 2010

BONDÁR 2015

BONDÁR-RACZKY 2009

FÁBIÁN 2014

FÁBIÁN et al. 2015

FURHOLT 2008a

FURHOLT 2008b
$=$ J. BANNER: Die Péceler Kultur. ArchHung 35. Budapest 1956.

= P. BIEHL-R. GLeSER: Theorien und Methoden der Stilanalyse. In: M. Heinz-K. H. Eggert-U. Veit (Hrsg.): Zwischen Erklären und Verstehen? Beiträge zu den erkenntnistheoretischen Grundlagen archäologischer Interpretation 2. Münster-New York-München-Berlin 2003, 149-174.

= M. BONDÁR: Újabb adatok a badeni kultúra temetkezéseihez (Neuere Beiträge zu Bestattungen der Badener Kultur). ZalaiMúz 1 (1987) 47-58.

= M. BONDÁR: Thoughts on continuity (The Baden culture). Antaeus 19/20 (1990/1991) 33-40.

= M. BonDÁR: A badeni kultúra kutatási helyzete Magyarországon. Vázlat (Der Forschungsstand der Badener Kultur in Ungarn. Abriss). MFMÉ-StudArch 8 (2002) 7-30.

= M. BONDÁR: The cemetery. In: BONDÁR-RACZKY 2009, 11-302.

= M. BONDÁR: Catalogue. In: BondÁR-RACZKY 2009, 31-196.

= M. BONDÁR: Funeral rites in the Budakalász cemetery. In: BONDÁR-RACZKY 2009, 197-244.

= M. BONDÁR: Grave goods. In: BONDÁR-RACZKY 2009, 245-300.

= M. BondÁR: The Late Copper Age settlement at Nagyút-Göbölyjárás II. Questions on the periodisation of the Baden culture. Antaeus 31-32 (2010) 303-374.

= M. Bondár: The Late Copper Age Cemetery at Pilismarót-Basaharc. István Torma's excavations (1967, 1969-1972). Budapest 2015.

= M. BonDÁR-P. RACZKY (eds): The Copper Age Cemetery of Budakalász. Budapest 2009.

= Sz. FÁBIÁN: A badeni kultúra településtörténete a dél-balatoni régióban az újabb kutatási eredmények alapján [Siedlungsgeschichte der Badener Kultur auf Grund der neueren Forschungsergebnisse in der südlichen Balaton-Region]. Manuskript. Budapest 2014.

$=$ Sz. Fábián-A. Kreiter-T. Marton-A. Rajna-G. Serlegi: The thing. The "Protoboleráz" phenomenon. In: Silesia and Related Areas from 46th to 36th centuries BC. Chronology and taxonomy. Special dedicated session 29th of May 2015. XIX. Silesian Archaeological Symposium, Wrocław 27th-30th of May 2015.

$=$ M. FURHOLT: Pottery, cultures, people? The European Baden material re-examined. Antiquity 82 (2008) 617-628.

= M. FuRHOLT: Culture history beyond cultures: The case of the Baden complex. In: M. FurholtM. Szmyt-A. Zastawny (eds): The Baden Complex and the Outside World. Proceedings of the 12th Annual Meeting of the EAA 2006, Cracow. Bonn 2008, 13-24. 
FuRHOLT 2009

FURHOLt 2011

FURHOLT 2013

FURHOLT-STOCKHAMMER 2008

GLESER 1995

HAMILTON 2015

HANSEN 2014

HORVÁTH 2011a

HORVÁTH 2011b

IHM 1983

KALICZ 1991

KALICZ 1999

KOREK 1951

MEISENHEIMER 1989

MRT 7

NAGY 2010

NAKOINZ-HINZ 2015

NĚMEJCOVÁ-PAVÚKOVÁ 1964

NĚMEJCOVÁ-PAVÚKOVÁ 1974

NĚMEJCOVÁ-PAVÚKOVÁ 1979

NĚMEJCOVÁ-PAVÚKOVÁ 1981

NĚMEJCOVÁ-PAVÚKOVÁ 1984

NEUSTUPNÝ 1973
$=$ M. FurhOLT: Die nördlichen Badener Keramikstile im Kontext des mitteleuropäischen Spätneolithikums (3650-2900 v. Chr.). Bonn 2009.

= M. Furholt: Materielle Kultur und räumliche Strukturen sozialer Identität im 4. und 3. Jt. v. Chr. in Mitteleuropa. Eine methodische Skizze. In: S. Hansen-J. Müller (Hrsg.): Sozialarchäologische Perspektiven: Gesellschaftlicher Wandel 5000-1500 v. Chr. zwischen Atlantik und Kaukasus. Internationale Tagung 15.-18. Oktober 2007 in Kiel. Mainz 2011, 243-267.

= M. Furholt: Die nördlichen Badener Keramikstile im Kontext des mitteleuropäischen Spätneolithikums (3650-2900 v. Chr.). ArchInf 29/1-2 (2006) 235-242.

= M. Furholt-Ph. Stockhammer: Wenn stumme Dinge sprechen sollen: Gedanken zu semiotischen Ansätzen in der Archäologie. In: M. Butter-R. Grundman-Ch. Sanchez (Hrsg.): Zeichen der Zeit. Interdisziplinäre Perspektiven zur Semiotik. Frankfurt/Main-Berlin-Bern-Bruxelles-New YorkOxford-Wien 2008, 59-71.

= R. GLESER: Die Epi-Rössener Gruppen in Südwestdeutschland. Untersuchungen zur Chronologie, stilistischen Entwicklung und kulturellen Einordnung. Saarbrücker Beiträge zur Altertumskunde 61. Bonn 1995.

= D. HAMILTON: The Pilismarót-Basaharc cemetery: Radiocarbon dating and Bayesian modelling. In: BONDÁR 2015, 349-352.

= S. HANSEn: Relative Chronologie. In: D. Mölders-S. Wolfram (Hrsg.): Schlüsselbegriffe der prähistorischen Archäologie. Münster 2014, 251-403.

= T. HoRvÁTH: A késő rézkor időszaka más szemszögből: tipokronológiai megfigyelések a Balatonőszöd-Temetői-dűlői késő rézkori boleráz/baden település leletanyagán [Die Zeit der Spätkupferzeit aus anderen Perspektiven: Typochronologische Beobachtungen auf Grund des Fundmaterials der Badener Siedlung von Balatonőszöd-Temetői-Flur]. Gesta 10 (2011) 3-135.

= T. HorvátH: A Boleráz, Baden és Kostolac kultúrák kronológiai és térbeli helyzete és interkulturális kapcsolatai (The Boleráz, Baden and Kostolac Cultures in the Late Copper Age and Early Bronze Age: Their chronological and spatial distribution and intercultural connections). Specimena Electronica Antiquitatis 12. Budapest 2011.

= P. IHM: Korrespondenzanalyse und Seriation. ArchInf 6/1 (1983) 8-21.

= N. KaLICZ: Beiträge zur Kenntnis der Kupferzeit im ungarischen Transdanubien. In: J. Lichardus (Hrsg.): Die Kupferzeit als historische Epoche I-II. Symposium Saarbrücken und Otzenhausen 6.-13. 11. 1988. Saarbrücker Beiträge zur Altertumskunde 55. Bonn 1991, 347-387.

= N. KALICZ: A késő rézkori Báden kultúra temetője Mezőcsát-Hörcsögösön és Tiszavasvári-Gyepároson (Das Gräberfeld der spätkupferzeitlichen Badener Kultur in Mezöcsát-Hörcsögös und Tiszavasvári-Gyepáros). HOMÉ 37 (1999) 57-101.

= J. KOREK: A badeni kultúra temetője Alsónémedin (The cemetery of the Baden culture at Alsónémedi). ActaArchHung 1 (1951) 41-66.

= M. MEISENHEIMER: Das Totenritual, geprägt durch Jenseitsvorstellungen und Gesellschaftsrealität. Theorie des Totenrituals eines kupferzeitlichen Friedhofs zu Tiszapolgár-Basatanya (Ungarn). BAR IntSer 475. Oxford 1989.

= I. Dinnyés-K. KõváRI-Zs. Lovag-S. TetTAmanti-J. TopáL-I. Torma: Magyarország régészeti topográfiája 7. Pest megye régészeti topográfiája. XIII/1: A budai és szentendrei járás [Archäologische Topographie Ungarns 7]. Budapest 1986.

= B. NAGY: Gräberfeld der Badener Kultur in Balatonlelle-Felső Gamász. Antaeus 31-32 (2010) 375-498.

= O. NAKoInZ-M. HinZ: Modelle in der Archäologie. In: B. Thalheim-I. Nissen (Hrsg.): Wissenschaft \& Kunst der Modellierung. Kieler Zugang zur Definition, Nutzung und Zukunft. Berlin-Boston 2015, 219-249.

= V. NĚMEJCOVÁ-PAVÚKOVÁ: Sídlisko bolerázskeho typu v Nitriánsky Hrádku (Siedlung der BolerázGruppe in Nitriánsky Hrádok). SlA 12/1 (1964) 163-268.

= V. NĚMejcovÁ-PavúKovÁ: Beitrag zum Kennen der Postbolerazentwicklung der Badener Kultur. S1A 22 (1974) 237-360.

= V. NĚMEJCOVÁ-PAVÚKOvÁ: Počiatky bolerázskej skupiny na Slovensku (Die Anfänge der BolerázGruppe in der Slowakei). SlA $27 / 1$ (1979) 17-55.

= V. NĚMEJCOVÁ-PAVÚKOVÁ: Načrt periodizácie badenskej kultúrý a jej chronologických vzt'ahov $\mathrm{k}$ juhovýchodnej Európe (An outline of the periodical system of Baden culture and its chronological relations to Southeast Europe). S1A 29/2 (1981) 261-296.

= V. NĚMEJCOVÁ-PAVÚKOVÁ: K problematike trnavia a konca bolerázskej skupiny na Slovensku (Zur Problematik von Dauer und Ende der Boleráz-Gruppe in der Slowakei). S1A 32 (1984) 75-146.

= E. NeustupnÝ: Die Badener Kultur. In: B. Chropovsky (Hrsg.): Symposium über die Entstehung und Chronologie der Badener Kultur. Bratislava 1973, 317-352. 
NEVIZÁNSKY 1985

NEVIZÁNSKY 2004

NEVIZÁNSKY et al. 1995/1996

PETRIE 1899

RAJNA 2011

SACHßE 2005

SACHßE 2008

SACHßE 2009

SACHßE 2010

SIKLÓSI 2009

SófALVI 2004

SOPRONI 1956

STEHLI 1973

StEHLI-ZimMERMANN 1980

STROBEL 1996

Točík 1987

TORMA 1969

TORMA 1973

WILD et al. 2001

ZALAI-GAÁL 2007a

ZALAI-GAÁL 2007b

ZALAI-GAÁL 2014

ZALAI-GAÁL 2015a

ZALAI-GAÁL 2015b

ZALAI-GAÁL 2016
= G. NEVIZÁNSKY: Grabfunde und Überbauerscheinungen der Träger der Badener Kultur im zentralen Gebiet des Karpatenbeckens. SlA 33 (1985) 249-270.

= G. NevizÁNSKY: Amphoren der Badener Kultur. In: J. Bátora-V. Furmánek-L. Veliačik (Hrsg.): Einflüsse und Kontakte alteuropäischer Kulturen. Festschrift für Jozef Vladár zum 70. Geburtstag. Nitra 2004, 57-73.

= G. NEVIZÁNSKY-J. JAKAB-O. OŽĽÁNI: Ein Brandgräberfeld der Badener Kultur in Malá nad Hronom. Sastuma 4/5 (1995/1996) 251-272.

=W. M. F. PETRIE: Sequences in prehistoric remains. The Journal of the Anthropological Institute of Great Britain and Ireland 29 (1899) 295-301.

= A. RAJNA: Az Abony 49. lelőhely protoboleráz-kori leletei és interpretációs lehetőségei [Die Funde der Protoboleráz-Zeit des Fundortes Abony 49 und die Möglichkeiten ihrer Interpretation]. StComit 31 (2011) 96-124.

$=\mathrm{C}$. SACHßE: Untersuchungen zu den Bestattungssitten der Badener Kultur in ihrem räumlichen und zeitlichen Umfeld. Diss. Univ. Heidelberg 2005.

= C. SACHßE: Baden cultural identities? Later Copper Age funerals reviewed. In: M. Furholt-M. Szmyt-A. Zastawny (eds): The Baden Complex and the Outside World. Proceedings of the 12th Annual Meeting of the EAA in Cracow, 19th-24th September 2006. Bonn 2008, 49-68.

$=$ C. SACHßE: Begraben, geopfert oder entsorgt? Sonderbestattungen während der Kupferzeit im westlichen Karpatenbecken. In: Vorträge des 27. Niederbayerischen Archäologentages. Rahden/Westf. 2009, 145-177.

= C. SACHßE: Untersuchungen zu den Bestattungssitten der Badener Kultur. I: Text, Teil II: Katalog, Tafeln. UPA 179. Bonn 2010.

$=$ Zs. SIKLÓSI: Absolute and internal chronology of the Late Copper Age cemetery at Budakalász. In: BONDÁR-RACZKY 2009, 457-474.

= A. Sófalvi: Balatonlelle-Országúti-dűlő és Balatonlelle-Felső-Gamász. In: Sz. Honti et al.: A tervezett M7-es autópálya Somogy megyei szakaszának megelőző régészeti feltárása (2002-2003). Elözetes jelentés III. (The preceding archaeological excavations 2002-2003 of the M7 highway in Somogy county. Preliminary report III). SMK 16 (2004) 18-23.

= S. Soproni: Budakalász, Luppa csárda [Budakalász, Luppa Tscharda]. In: BANNER 1956, 111-128.

= P. STEHLI: Keramik. In: J.-P. Farrugia-R. Kuper-J. Lüning-P. Stehli: Der bandkeramische Siedlungsplatz Langweiler 2, Gemeinde Aldenhoven, Kreis Düren. Bonn 1973, 57-100.

= P. Stehli-A. Zimmermann: Zur Analyse neolithischer Gefäßformen. Archaeo-Physika 7 (1980) $147-177$.

= M. STROBEL: Ein Beitrag zur Gliederung der östlichen Linearbandkeramik. Versuch einer Merkmalanalyse. Sastuma 4-5 (1995-1996 [1996]) 9-98.

= A. Tоčík: Záchranný výskum v Lipovej-Ondrochove v roku 1980 (Rettungsgrabung in LipovaOndrochov im Jahre 1980). ŠtZ 23 (1987) 243-303.

= I. ToRmA: Adatok a bádeni (péceli) kultúra bolerázi csoportjának magyarországi elterjedéséhez (Contribution à l'historique de l'extension du groupe de Boleraz de la civilisation badoise). VMMÉ 8 (1969) 91-108

= I. Torma: Die Boleraz-Gruppe in Ungarn. In: B. Chropovský (Hrsg.): Symposium über die Entstehung und Chronologie der Badener Kultur. Symposium Male Vozokany 1969. Bratislava 1973, 483-512.

= E. M. Wild-P. Stadler-M. Bondár-S. Draxler-H. Friesinger-W. Kutschera-A. PrillerW. Rom-E. Ruttkay-P. SteiER: New chronological frame for the young Neolithic Baden culture in Central Europe (4th Millennium BC). Radiocarbon 43 (2001) 1057-1064.

= I. ZALAI-GAÁL: Von Lengyel bis Mórágy. Die spätneolithische Grabkeramik in Südtransdanubien aus den alten Ausgrabungen. I. Analyse. WMMÉ 29 (2007) 1-177.

= I. ZALAI-GAÁL: Zengővárkony - Svodín - Friebritz. Zu den chronologischen Beziehungen zwischen den territorialen Gruppen der Lengyel-Kultur aufgrund der Gräberfeldanalyse. In: J. KozłowsiP. Raczky (eds): The Lengyel, Polgár and Related Cultures in the Middle/Late Neolithic in Central Europe. Krakow 2007, 153-189.

= I. ZALAI-GAÁL: Die „Steinkupferzeit“ im westlichen Karpatenbecken (5. Jahrtausend v. Chr.). Manuskript. Budapest 2014

= I. ZALAI-GAÁL: „Der Anfang und das Ende“. Die Gräberfelder des kupferzeitlichen Polgár-Komplexes im östlichen Karpatenbecken. Manuskript. Budapest 2015.

= I. ZALAI-GAÁL: Streitfragen der frühkupferzeitlichen Forschungen im östlichen Karpatenbecken. Antaeus 33 (2015) 55-98.

= I. ZALAI-GAÁL: Zeiträume und Formenkreise - Zur Chronologie der kupferzeitlichen Nekropolen im östlichen Karpatenbecken. ActaArchHung 67 (2016) 31-98. 
ZALAI-GAÁL et al. 2014

ZeEB-LANZ 2003

ZeEb-LANZ 2006
= I. ZALAI-GAÁL-A. OsZTÁs-K. Somogyi: Zur relativen Chronologie der Lengyel-Kultur im westlichen Karpatenbecken. ActaArchHung 65 (2014) 285-334.

= A. ZeEB-LanZ: Keramikverzierungsstil als Kommunikationsmittel: Ein Beispiel aus dem frühen Jungneolithikum Südwestdeutschlands. In: U. Veit et al. (Hrsg.): Spuren und Botschaften: Interpretationen materieller Kultur. Münster 2003, 245-261.

= A. ZeEB-LanZ: Überlegungen zu Sozialaspekten keramischer Gruppen. Beispiele aus dem Neolithikum Südwestdeutschlands. In: S. Burgmeister-N. Müller-Scheeßel (Hrsg.): Soziale Gruppen kulturelle Grenzen. Münster 2006, 81-102. 